\title{
RNA promotes the formation of spatial compartments in the nucleus
}

Sofia A. Quinodoz ${ }^{1}$, Prashant Bhat ${ }^{1,2,5}$, Noah Ollikainen ${ }^{1,5}$, Joanna W. Jachowicz ${ }^{1,5}$, Abhik K.

Banerjee $^{1,3}$, Peter Chovanec ${ }^{1}$, Mario R. Blanco ${ }^{1}$, Amy Chow $^{1}$, Yolanda Markaki ${ }^{4}$, Kathrin Plath ${ }^{4}$, and Mitchell Guttman ${ }^{1 *}$

(1) Division of Biology and Biological Engineering, California Institute of Technology, Pasadena, CA 91125, USA

(2) David Geffen School of Medicine, University of California, Los Angeles, Los Angeles, CA 90095, USA

(3) Keck School of Medicine, University of Southern California, Los Angeles, CA 90089, USA

(4) Department of Biological Chemistry, University of California, Los Angeles, Los Angeles, CA 90095, USA

(5) These authors contributed equally to this work.

${ }^{*}$ To whom correspondence should be addressed. mguttman@caltech.edu (MG) 


\section{SUMMARY}

2 The nucleus is a highly organized arrangement of RNA, DNA, and protein molecules that are

3 compartmentalized within three-dimensional (3D) structures involved in shared functional and regulatory

4 processes. Although RNA has long been proposed to play a global role in organizing nuclear structure,

5 exploring the role of RNA in shaping nuclear structure has remained a challenge because no existing

6 methods can simultaneously measure RNA-RNA, RNA-DNA, and DNA-DNA contacts within 3D

7 structures. To address this, we developed RNA \& DNA SPRITE (RD-SPRITE) to comprehensively map

8 the location of all RNAs relative to DNA and other RNAs. Using this approach, we identify many RNAs

9 that are localized near their transcriptional loci (RNA-DNA) together with other diffusible ncRNAs

10 (RNA-RNA) within higher-order DNA structures (DNA-DNA). These RNA-chromatin compartments

11 span three major classes of nuclear functions: RNA processing (including ribosome biogenesis, mRNA

12 splicing, snRNA biogenesis, and histone mRNA processing), heterochromatin assembly, and gene

13 regulation. More generally, we identify hundreds of ncRNAs that form stable nuclear compartments in

14 spatial proximity to their transcriptional loci. We find that dozens of nuclear compartments require RNA

15 to guide protein regulators into these 3D structures, and focusing on several ncRNAs, we show that these

16 ncRNAs specifically regulate heterochromatin assembly and the expression of genes contained within

17 these compartments. Together, our results demonstrate a unique mechanism by which RNA acts to shape

18 nuclear structure by forming high concentration territories immediately upon transcription, binding to

19 diffusible regulators, and guiding them into spatial compartments to regulate a wide range of essential

20 nuclear functions. 


\section{INTRODUCTION}

23 The nucleus is spatially organized in three-dimensional (3D) structures that are important for various 24 functions including DNA replication, transcription, and RNA processing ${ }^{1-6}$. To date, genome-wide studies of nuclear organization have focused primarily on the role of $\mathrm{DNA}^{2,7,8}$, yet nuclear structures are known to contain multiple DNA, RNA, and protein molecules that are involved in shared functional and regulatory processes ${ }^{1-6}$. These include classical compartments like the nucleolus ${ }^{9}$ (which contains transcribed ribosomal RNAs and their processing molecules) and nuclear speckles ${ }^{10}$ (which contain nascent pre-mRNAs and mRNA splicing components), as well as the more recently described transcriptional condensates ${ }^{11,12}$ (which contain Mediator and RNA Polymerase II). Because the complete molecular architecture of the nucleus has not been globally explored, the full extent to which such nuclear compartments exist and contribute to nuclear function remains unknown. Even for the specific nuclear compartments that have been molecularly characterized, the mechanism by which RNA and protein molecules transition from diffuse locations throughout the nucleus into compartmentalized structures remains largely unknown.

Nuclear RNA has long been proposed to play a central role in shaping nuclear structure ${ }^{13-18}$. Initial experiments performed more than 30 years ago found that global disruption of RNA (using RNase) leads to large scale morphological deficits in the nucleus ${ }^{13}$. Over the past decade it has become clear that mammalian genomes encode thousands of nuclear-enriched ncRNAs ${ }^{19-21}$, several of which play critical roles in the regulation of essential nuclear functions ${ }^{22,23}$. These include ncRNAs involved in splicing of pre-mRNAs (snRNAs) ${ }^{24,25}$, cleavage and modification of pre-ribosomal RNAs (snoRNAs, Rnase MRP) ${ }^{26-}$ 28, 3'-end cleavage and processing of the non-polyadenylated histone pre-mRNAs (U7 snRNA) ${ }^{29-32}$, and transcriptional regulation (e.g. Xist ${ }^{33-35}$ and $7 \mathrm{SK}^{36-38}$ ). Interestingly, many of these functionally important ncRNAs localize within specific spatial compartments in the nucleus ${ }^{6,39,40}$. For example, snoRNAs and the 45S pre-ribosomal RNA localize within the nucleolus ${ }^{9,41-43}$, Xist localizes on the inactive $\mathrm{X}$ chromosome (Barr body) $)^{35,44-46}$, and snRNAs and Malat1 localize within nuclear speckles ${ }^{10,47}$.

In each of these examples, multiple RNA, DNA, and protein components simultaneously interact within precise three-dimensional structures to coordinate specific nuclear functions. While the roles of these specific ncRNAs have been well studied, comprehensively mapping the localization patterns of most nuclear ncRNAs relative to other RNAs and DNAs in 3D space remains a challenge because no existing 
51 method can simultaneously measure RNA-RNA, RNA-DNA, and DNA-DNA contacts within 3D structures. As a result, it is unclear: (i) which specific RNAs might be involved in nuclear $\operatorname{organization}^{16,18,48}$, (ii) which specific nuclear compartments are dependent on RNA, and (iii) what mechanisms RNA might utilize to organize nuclear structures.

Microscopy is currently the only way to relate RNA and DNA molecules in 3D space. However, this approach is limited to examining a small number of simultaneous interactions and therefore requires $a$ priori knowledge of which RNAs and nuclear structures to explore. An alternative approach is genomic mapping of RNA-DNA contacts using proximity-ligation methods ${ }^{49-53}$. While these approaches can provide genome-wide pairwise maps of RNA-DNA interactions, they do not provide information about the 3D organization of these molecules in the nucleus. Moreover, we recently showed that proximityligation methods can fail to identify pairwise contacts between molecules that are organized within nuclear compartments because these methods only identify interactions where components are close enough in space to be directly ligated ${ }^{54}$. Consistent with this observation, existing RNA-DNA proximity-ligation methods fail to identify known RNA-DNA contacts that are contained within various well-established nuclear bodies, such as nucleoli, histone locus bodies (HLBs), and Cajal bodies ${ }^{50-53}$.

We recently developed SPRITE, a proximity-ligation independent method that utilizes split-and-pool barcoding to generate accurate, comprehensive, and multi-way 3D spatial maps of the nucleus across a wide range of distances ${ }^{54}$. Importantly, we showed that this approach can accurately map the spatial organization of DNA arranged around two nuclear bodies - nucleoli and nuclear speckles ${ }^{54}$. However, our original version of the technique could not detect the vast majority of ncRNAs - including low abundance ncRNAs known to organize within several well-defined nuclear structures - thereby precluding a comprehensive map of RNA localization within the nucleus. Here, we introduce a dramatically improved method, RNA \& DNA SPRITE (RD-SPRITE), which enables simultaneous and high-resolution measurements of thousands of RNAs - including low abundance RNAs such as nascent pre-mRNAs and ncRNAs - relative to all other RNA and DNA molecules in 3D space. Using this approach, we identify hundreds of RNA-containing nuclear structures that are each largely organized within higher-order structures around shared regulatory targets. We demonstrate that many ncRNAs form high concentration territories within defined spatial compartments throughout the nucleus. Many of these ncRNAs bind to diffusible ncRNAs and proteins and act to guide their localization within these nuclear compartments. 
81 these spatial compartments. Together, our results demonstrate a privileged role for RNA in the formation

82 of nuclear compartments that are involved in a wide range of essential nuclear functions including RNA

83 processing, heterochromatin assembly, and gene regulation.

\section{RESULTS}

RD-SPRITE generates accurate maps of higher-order RNA and DNA contacts throughout the cell

Exploring the role of RNA in shaping nuclear structure has remained a challenge because no existing genomic method can simultaneously measure RNA-RNA, RNA-DNA, and DNA-DNA contacts within 3D structures. To address this, we developed RNA \& DNA SPRITE (RD-SPRITE) to comprehensively map the location of all RNAs relative to DNA and other RNAs. Specifically, we improved the efficiency of the RNA-tagging steps of our SPRITE method ${ }^{55}$ to enable detection of all classes of RNA - from highly abundant ribosomal RNAs and snRNAs to less abundant lncRNAs and individual nascent pre-mRNAs

(Supplemental Note 1). Briefly, our approach works as follows: (i) RNA, DNA, and protein contacts are crosslinked to preserve their spatial relationships in situ, (ii) cells are lysed and the contents are fragmented into smaller crosslinked complexes, (iii) DNA and RNA within each complex are tagged with a sequencespecific adaptor, (iv) barcoded using an iterative split-and-pool strategy to uniquely assign a shared barcode to all DNA and RNA components contained within a crosslinked complex, (v) DNA and RNA are sequenced, and (vi) all reads sharing identical barcodes are merged into a group that we refer to as a SPRITE cluster (Figure 1A, Supplemental Figure 1A, see Methods). Accordingly, RD-SPRITE enables simultaneous mapping of multi-way DNA-DNA, RNA-DNA, and RNA-RNA contacts in the same experiment. Because RD-SPRITE does not rely on proximity ligation, it can detect multiple RNA and DNA molecules that simultaneously associate within the nucleus (referred to as higher-order structures).

We performed RD-SPRITE in an F1 hybrid female mouse ES cell line that was engineered to induce Xist from a single allele (see Methods). We sequenced these libraries on a NovaSeq S4 run to generate $\sim 8$ billion reads corresponding to $\sim 720$ million SPRITE clusters (Supplemental Figure 1C). We confirmed that we accurately identify RNA- and DNA-specific reads (Supplemental Figure 1A-B) and that the data measure bona fide RNA interactions - including well-described RNA-DNA and RNA-RNA contacts not only in the nucleus, but throughout the cell. 
109 First, we explored RNA-DNA contacts captured in our data and compared their interactions to those of 110 several ncRNAs that have been previously mapped to chromatin that reflect a range of known cis and 111 trans localization patterns. Specifically, we observed strong enrichment of: (i) Xist over the inactive X 112 (Xi), but not the active $X$ chromosome $(\mathrm{Xa})^{46,56}$ (Figure 1B, Supplemental Figure 1D); (ii) Malat1 and 113 U1 over actively transcribed RNA Polymerase II genes ${ }^{57,58}$ (Figure 1B); and (iii) telomerase RNA 114 component (Terc) over telomere-proximal regions of all chromosomes (Supplemental Figure 1E) $)^{59,60}$.

115 Second, we explored known RNA-RNA contacts that occur in different locations in the cell. For example, 116 we observed a large number of contacts between translation-associated RNAs in the cytoplasm, including 117 all RNA components of the ribosome (5S, 5.8S, 18S, 28S) and $\sim 8000$ individual mRNAs (exons), but not 118 with pre-mRNAs (introns). Conversely, we observed many contacts between the small nuclear RNA 119 (snRNA) components of the spliceosome (e.g. U1, U2, U4, U5, U6) in the nucleus and individual pre120 mRNAs (Figure 2A).

121 Together, these results demonstrate that RD-SPRITE accurately measures known RNA-DNA and RNA122 RNA localization patterns in the nucleus and cytoplasm. While we focus primarily on RNA localization 123 within the nucleus, we note that RD-SPRITE can also be utilized to study RNA compartments beyond the 124 nucleus ${ }^{61-63}$.

Non-coding RNAs localize in higher-order spatial compartments in the nucleus

127 Because RD-SPRITE generates comprehensive structure maps of RNA and DNA in the nucleus, we 128 explored which specific RNAs localize within higher-order compartments. To do this, we mapped all 129 RNA-RNA and RNA-DNA interactions genome-wide. Specifically, we identified several sets of RNA 130 and genomic DNA regions that display high contact frequencies within their corresponding set, but low 131 contact frequencies with molecules contained within distinct sets. We refer to the RNA and DNA 132 molecules within an interacting set as an RNA-DNA hub (Figure 2B, Supplemental Figure 2A-B). Using 133 a combination of RNA FISH (to visualize RNAs) and immunofluorescence (to visualize different cellular 134 compartments) we confirmed that RNAs within a hub co-localize (Supplemental Figure 2C), while 135 RNAs in distinct hubs localize to different regions of the cell (Supplemental Figure 2D). Using multi136 way SPRITE clusters, we found that these RNA-DNA hubs form higher-order structures in the nucleus 
137 that contain multiple RNAs and genomic DNA regions that are organized in 3D space (Figure 3B) around 138 shared regulatory functions, which we describe below.

\section{Non-coding RNAs form processing hubs around genomic DNA encoding their nascent targets}

141 We first explored the RNA-DNA hubs corresponding to several nuclear compartments associated with 142 RNA processing. Biochemical approaches have revealed that RNA processing generally involves direct 143 hybridization between various diffusible trans-acting ncRNAs (e.g. splicing snRNAs) and their nascent 144 target RNA substrates (e.g. pre-mRNAs). However, these approaches study a few molecules at a time and 145 require a priori knowledge of which molecular components comprise specific nuclear bodies. Using RD146 SPRITE to measure the higher-order organization of RNA and DNA molecules within each processing 147 hub, we examined: (i) the RNA components in these hubs (RNA-RNA interactions), (ii) the location of 148 each RNA relative to the DNA loci from which their nascent target substrate RNAs are transcribed (RNA149 DNA interactions), and (iii) whether RNA processing occurs at individual locations or whether multiple 150 DNA loci come together in 3D space (DNA-DNA interactions).

151 (i) $n c R N A s$ involved in ribosomal RNA processing organize within a $3 D$ compartment containing 152 transcribed ribosomal RNA genes. We identified a hub that includes the $45 \mathrm{~S}$ pre-ribosomal RNA (pre153 rRNA), RNase MRP, and dozens of snoRNAs that are involved in ribosomal RNA biogenesis (Figure 154 2B, Supplemental Figure 2A, 3A). rRNA is transcribed as a single 45S precursor RNA and is cleaved 155 by RNAse MRP and modified by various snoRNAs to generate the mature $18 \mathrm{~S}, 5.8 \mathrm{~S}$, and $28 \mathrm{~S}$ rRNAs ${ }^{64-}$ $156{ }^{66}$. We found that all of these RNAs diffuse through the nucleus and localize at genomic locations that are 157 proximal to ribosomal DNA repeats that encode the $45 \mathrm{~S}$ pre-rRNA and other genomic regions that we 158 previously showed to organize around the nucleolus ${ }^{54}$ (Figure 3A, 3C, see Methods). We explored the 159 DNA-DNA interactions that occur within SPRITE clusters containing multiple nucleolar hub RNAs (45S 160 pre-rRNA and snoRNAs, $\geq 4$-way contacts) (Figure 3B), and observed that these RNAs and the multiple 161 genomic DNA regions encoding 45S pre-RNAs are organized together in 3D space (Figure 3D, 162 Supplemental Figure 3B, see Methods). Our results demonstrate that the nascent 45S pre-rRNA is 163 enriched near the DNA loci from which it is transcribed. In this way, 45S pre-rRNA (which is known to 164 directly interact with snoRNAs and RNase MRP ${ }^{22,64}$ ) may act to concentrate these diffusible trans-acting 165 regulatory ncRNAs that are responsible for ribosome biogenesis into the nucleolar compartment (Figure 
166 3E). Consistent with this, inhibition of $45 \mathrm{~S}$ pre-rRNA transcription has been shown to disrupt nucleolar 167 organization $^{9,42,43}$.

(ii) ncRNAs involved in mRNA splicing are spatially concentrated around transcribed Pol II genes. We identified a hub that contains nascent pre-mRNAs along with all of the major (e.g. U1, U2, U4, U5, U6) and minor (U11, U12) spliceosomal ncRNAs and other ncRNAs associated with transcriptional regulation

171 and mRNA splicing (e.g. 7SK and Malat1) (Figure 2A-B). Nascent pre-mRNAs are known to be directly 172 bound and cleaved by spliceosomal RNAs to generate mature mRNA transcripts ${ }^{24,67}$. Although splicing 173 can occur co-transcriptionally $24,68,69$, it has been unclear how spliceosomal RNAs are organized in the 174 nucleus relative to target pre-mRNAs and genomic DNA ${ }^{69-74}$. We found that the spliceosomal hub RNAs 175 localize to genomic DNA regions containing actively transcribed Pol II genes (Pearson $r=0.84-0.90$, 176 Figure 3A, 3F, Supplemental Figure 3C). We explored DNA-DNA contacts within SPRITE clusters containing individual and multiple spliceosomal hub RNAs ( $\geq 2$ distinct RNAs, $\geq 4$-way RNA-DNA contacts) and observed that these RNAs and genomic DNA regions form preferential intra- and interchromosomal contacts that are organized together in 3D space (Figure 3G, Supplemental Figure 3D). These results demonstrate that spliceosomal RNAs are spatially organized around clusters of actively transcribed Pol II genes and their associated nascent pre-mRNAs (Figure 3H). Because nascent premRNAs are enriched in spatial proximity to their transcriptional locus and are known to directly hybridize to splicing $\mathrm{RNAs}^{75,76}$, nascent pre-mRNAs may act to recruit these diffusible trans-acting regulatory ncRNAs into high spatial concentrations near their co-transcriptional targets.

(iii) ncRNAs involved in SnRNA biogenesis are spatially organized around snRNA gene clusters. We identified a hub containing several annotated small Cajal body-associated RNAs (scaRNAs), two previously unannotated scaRNAs, and several small nuclear RNAs (snRNAs) (Figure 2B, Supplemental

Figure 4D, see Methods). snRNAs are Pol II transcripts produced from multiple locations throughout the genome that undergo 2'O-methylation and pseudouridylation before functionally acting as components of the spliceosome at thousands of nascent pre-mRNA targets ${ }^{77-79}$; scaRNAs directly hybridize to snRNAs to guide these modifications ${ }^{80-82}$. We found that scaRNAs are highly enriched at discrete genomic regions containing multiple snRNA genes in close linear space (Figure 4A). Despite being separated by large genomic distances, these DNA regions form long-range contacts in SPRITE clusters containing scaRNAs ( $\geq 3$-way RNA-DNA contacts) (Figure 4B, Supplemental Figure 4E). In fact, we observe that these scaRNAs, snRNAs, and the distal DNA loci from which the snRNAs are transcribed simultaneously 
interact within higher-order SPRITE clusters, demonstrating that all of these components interact within a 3D hub in the nucleus (Supplemental Figure 4G). Because snRNAs are enriched in spatial proximity to their transcriptional loci and are known to directly hybridize to diffusible scaRNAs ${ }^{81,82}$, nascent snRNAs may act to recruit and concentrate scaRNAs within this compartment to enable snRNA biogenesis and modification ${ }^{83}$ (Figure 4E). We note that this snRNA biogenesis hub may be similar to Cajal bodies, which have been noted to contain snRNA genes and scaRNAs ${ }^{76,84-87}$ (see Supplementary Note 2).

(iv) The histone processing $U 7 \operatorname{snRNA}$ is spatially enriched around histone gene loci. We identified a hub containing the U7 snRNA and various histone mRNAs (Figure 2B). Unlike most pre-mRNAs, histone pre-mRNAs are not polyadenylated; instead their 3'ends are bound and cleaved by the U7 snRNP complex to produce mature histone mRNAs ${ }^{31,88}$. This process is thought to occur within nuclear structures called Histone Locus Bodies (HLBs) ${ }^{32,80}$. We observed that the U7 snRNA localizes at genomic DNA regions containing histone mRNA genes, specifically, at two histone gene clusters on chromosome 13 (Figure 4A). To determine whether the U7 snRNA, histone gene loci, and nascent histone pre-mRNAs form a 3D spatial compartment, we generated DNA-DNA interaction maps from U7 snRNA-containing clusters ( $\geq 3$ way RNA-DNA contacts) and observed long-range DNA contacts between the two histone gene clusters on chromosome 13 (Figure 4C, Supplemental Figure 4F). Because histone pre-mRNAs are present at high concentrations near their transcriptional loci and directly bind to U7, they may act to recruit these trans-associating ncRNAs into the HLB compartment (Figure 4F). Consistent with this model, previous studies have shown that histone pre-mRNAs are sufficient to seed the formation of the HLB and that the U7 binding site on the histone pre-mRNA is required for HLB formation ${ }^{80,89,90}$. Additionally, we observed that scaRNAs also localize to these histone gene clusters, form higher-order DNA interactions, and are adjacent to the HLB in the nucleus (Figure 4A,D, Supplemental Figure 4C,H). This is consistent with previous observations that HLBs and Cajal bodies are often found adjacent to each other in the nucleus ${ }^{80,85}$ and tethering histone pre-mRNAs to chromatin can also lead to recruitment of components of Cajal bodies $^{89}$.

These results demonstrate that RD-SPRITE comprehensively and simultaneously maps RNA and DNA molecules comprising several well-defined RNA processing hubs. Specifically, in all of these cases, we observe that: (i) nascent substrate RNAs interact with diffusible trans-associating ncRNAs (RNA-RNA), (ii) these nascent RNAs are localized near their DNA loci (RNA-DNA), and (iii) multiple DNA loci 
containing these nascent transcripts come together in 3D space (DNA-DNA). Using SPRITE, we

227 previously detected higher order DNA organization around two landmark nuclear bodies - nucleoli and nuclear speckles ${ }^{54}$; our results now show that higher-order organization of multiple DNA loci (DNADNA) around shared targets is a general principle of RNA processing that includes ribosomal RNA, mRNA, snRNA, and histone mRNA biogenesis.

\section{Satellite-derived ncRNAs organize HP1 localization at inter-chromosomal hubs}

233 In addition to RNA processing, we also identified a hub containing ncRNAs transcribed from minor and 234 major satellite DNA regions within centromeric and pericentromeric regions, respectively (Figure 2B). 235 We found that these ncRNAs localize primarily over centromere-proximal regions (Figure 5A-B, 236 Supplemental Figure 5B) and organize into higher-order structures containing these ncRNAs and multiple centromere-proximal regions from different chromosomes that interact simultaneously (Figure 5C, Supplemental Figure 5A). This suggests that these RNAs demarcate a nuclear body where centromeric regions of chromosomes interact with each other. To confirm this, we performed DNA FISH on the major and minor satellite DNA and observed higher-order structures where multiple centromeres from distinct chromosomes interact simultaneously ${ }^{91,92}$ (Figure 5D).

Higher-order organization of centromeric and pericentromeric DNA, often referred to as chromocenters, represent one of the most well-defined regions of heterochromatin assembly and are enriched for various heterochromatin enzymes and chromatin modifications, including the HP1 protein and H3K9me3 modifications $^{92}$. Previous studies have shown that global disruption of RNA by RNase A leads to disruption of HP1 localization at chromocenters ${ }^{91}$. However, RNAse A is not specific and can impact several structures in the nucleus, including nucleoli ${ }^{93}$. Because major and minor satellite-derived ncRNAs localize exclusively within centromere-proximal structures, we hypothesized that these ncRNAs might be important for HP1 localization. To test this, we used a locked nucleic acid (LNA) antisense oligonucleotide (ASO) to degrade either the major or minor satellite RNAs (see Methods). We found that disruption of either the major or minor satellite RNA leads to depletion of HP1 proteins over these centromere-proximal structures and altered chromocenter organization (Figure 5E-F, Supplemental

Figure 5C-F, see Supplemental Note 4). Our results demonstrate that major and minor satellite RNAs are enriched within spatial proximity of their transcriptional loci and are required to recruit HP1 into 
centromere-proximal nuclear compartments to maintain higher-order centromeric heterochromatin

256 (Figure 5G). Consistent with this, previous studies have shown that disruption of the major satellitederived RNA prior to the formation of chromocenters during preimplantation development leads to loss of chromocenter formation, lack of heterochromatin formation, and embryonic arrest ${ }^{94-96}$.

\section{Hundreds of non-coding RNAs localize in spatial proximity to their transcriptional loci}

Thousands of nuclear-enriched ncRNAs are expressed in mammalian cells, but only a handful have been mapped on chromatin. We mapped $\sim 650$ lncRNAs in ES cells and observed a striking difference in chromatin localization between these lncRNAs and mature mRNAs (Figure 6A, Supplemental Figure are strongly enriched within 3D proximity of their transcriptional loci (Figure 6B-D, Supplemental Figure 6C, see Methods). This is in contrast to mature mRNAs, which are depleted near their transcriptional loci and at all other genomic locations (Supplemental Figure 6D-E). We observed a similar lack of chromatin enrichment for a subset of lncRNAs (enrichment score $<0$ ), including Norad which is known to localize and function in the cytoplasm ${ }^{97}$ (Figure 6A-B). Additionally, not all lncRNAs with high chromatin enrichment are restricted to the 3D compartment around their locus. For example, the Malat1 lncRNA is strongly enriched on chromatin but localizes broadly across all chromosomes (Figure 6A-B, Supplemental Figure 6C). These data demonstrate that the vast majority of lncRNAs in ES cells localize exclusively in spatial proximity to their transcriptional loci and do not diffuse to other locations in the nucleus or cytoplasm (Figure 6D-E).

This exclusive localization pattern could reflect the formation of a stable RNA-enriched nuclear compartment or simply represent an unstable RNA product that is transiently associated with its transcriptional locus prior to being rapidly degraded (Supplemental Figure 6A). To exclude the possibility that these represent unstable RNA products, we explored the expression of these lncRNAs after treating cells with flavopiridol (FVP), a drug that runs off elongating Pol II and prevents re-initiation of transcription ${ }^{98}$. We explored a previously published global RNA sequencing experiment performed after 50 minutes of treatment with FVP in $\mathrm{mES}$ cells ${ }^{99}$. Consistent with previous reports ${ }^{100}$, we found that virtually all lncRNAs were dramatically more stable than nascent pre-mRNAs and comparable to the stability of mature mRNAs (Figure 6F). To confirm this, we performed RNA FISH for 4 lncRNAs, 6 
nascent pre-mRNAs (introns), and 1 mature mRNA (exons) in untreated cells and upon FVP treatment (see Methods). We found that all of the lncRNAs form stable nuclear foci that are retained upon transcriptional inhibition (Figure 6G, Supplemental Figure 6F). In contrast, all nascent pre-mRNA foci are lost upon transcriptional inhibition, even though we observe no impact on their mature mRNA products (Figure 6G).

Together, these results demonstrate that many lncRNAs form high concentration territories within defined nuclear compartments. While their complete molecular composition and functional relevance remain unclear, our results demonstrate that these RNA-compartments are widespread and can demarcate local spatial territories throughout the nucleus (Figure 6E).

\section{Non-coding RNAs guide regulatory proteins to nuclear compartments to regulate gene expression}

Because hundreds of lncRNAs are enriched in spatial compartments throughout the nucleus, we explored how RNA localization might impact protein localization within these compartments. Recently, we and others showed that SHARP (also called Spen) directly binds Xist ${ }^{101-105}$ and recruits the HDAC3 histone deacetylase complex to the $\mathrm{X}$ chromosome to silence transcription ${ }^{103,106,107}$ (Supplemental Figure 7A). To explore the nuclear localization of SHARP more globally, we performed super-resolution microscopy and found two types of SHARP localization: low-level diffuse localization throughout the nucleus and compartmentalized localization within dozens of well-defined, high-intensity, foci throughout the nucleus ( 50-100 foci/nucleus) (Figure 7B, see Supplemental Video 1). To determine whether the compartmentalized SHARP foci are dependent on RNA, we deleted the RNA binding domains $(\Delta R R M)$ from the protein (Figure 7A) and found that it led to loss of all compartmentalized SHARP foci without affecting diffusive localization of the protein throughout the nucleus (Figure 7B, see Supplemental Video 2). These results demonstrate that RNA is required for SHARP localization to dozens of compartments throughout the nucleus.

To explore how these ncRNA-mediated nuclear compartments might act to regulate gene expression, we purified SHARP and mapped its interactions with specific RNAs. We identified strong binding to several RNAs, including a $\sim 600$ nucleotide region at the 5' end of Kcnq1ot1, a lncRNA associated with the

311 pediatric Beckwith-Wiedemann overgrowth syndrome ${ }^{108}$ (see Methods, Figure 7C). We found that 
312 Kcnq1ot1 localizes within the topologically associating domain (TAD) that contains all of the known 313 paternally-imprinted genes (Cdkn1c, Slc22a18, Phlda2) ${ }^{108,109}$, but excludes other genes that are close in 314 linear space in the genome (e.g. Cars, Nap114, Figure 7D, Supplemental Figure 7G). We confirmed that 315 downregulation of Kcnq1ot1 using CRISPRi leads to upregulation of these target genes and, conversely, 316 bi-allelic induction of Kcnq1ot1 expression leads to silencing of these imprinted target genes. In both 317 cases, there was no impact on the genes outside of this Kcnq1ot1-associated domain (Figure 7E-F, 318 Supplemental Figure 7C).

319 To explore whether SHARP binding to the Kcnq1ot1 RNA is essential for transcriptional silencing in this 320 compartment, we deleted the SHARP binding site on Kcnq1ot1 ( $\Delta$ SBS) and observed upregulation of its 321 known target genes in two independent clones (Figure 7G). Because SHARP is known to recruit 322 HDAC3 ${ }^{103,106,107}$, we tested whether Kcnq1ot1-mediated silencing is dependent on histone deacetylase 323 activity by treating cells with a small molecule that inhibits HDAC activity (TSA). We observed a loss of 324 Kcnq1ot1-mediated silencing only at the genes it localizes to, but not neighboring genes (Supplemental 325 Figure 7B). Together, these results demonstrate that Kcnq1ot1 localizes at a high concentration within 326 the TAD containing its transcriptional locus, binds directly to SHARP, and recruits SHARP and its 327 associated HDAC3 complex to silence transcription of genes within this nuclear compartment (Figure 328 7H).

329 In addition to Kcnqlot1, we identified several other lncRNAs that demarcate specific nuclear 330 compartments around their transcriptional loci containing their functional targets. For example: (i) Airn 331 localizes within a TAD containing its reported imprinted target genes ${ }^{110-112}$ but excludes other neighboring 332 genes in the genome (Supplemental Figure 7D,G). (ii) Pvt1 localizes to a TAD containing Myc and 333 multiple known enhancers of Myc (Supplemental Figure 7E,G) and has been shown to repress Myc 334 expression $^{113}$. (iii) Chaserr localizes within the TAD containing Chd2 (Supplemental Figure 7F) and has 335 been shown to repress Chd2 expression ${ }^{114,115}$.

336 These results demonstrate that the localization patterns of a ncRNA in 3D space guides the recruitment of 337 regulatory proteins to these specific nuclear compartments and highlights an essential role for these RNA338 mediated compartments in gene regulation. 


\section{DISCUSSION}

341 Our results demonstrate that RNAs localize within hundreds of compartments that occur throughout the 342 entire nucleus, and that RNA is required for the localization of specific diffusible proteins (HP1, SHARP) 343 and ncRNAs (e.g. U7, U1, snoRNAs, etc.) into dozens of compartmentalized structures. In all of these 344 cases, we observed a common theme where (i) specific RNAs localize at high concentrations in spatial 345 proximity to their transcriptional loci and (ii) diffusible ncRNA and protein molecules that bind to these 346 RNAs are enriched within these compartmentalized structures. These observations suggest a common 347 mechanism by which RNA can mediate nuclear compartmentalization: nuclear RNAs can form high concentration spatial territories close to their transcriptional loci ("seed"), bind to diffusible regulatory ncRNAs and proteins through high affinity interactions ("bind") and by doing so, act to dynamically change the spatial distribution of these diffusible molecules in the nucleus such that they are enriched within compartments composed of multiple DNA loci, regulatory and target RNAs, and proteins in 3D space ("recruit", Figure 8).

This mechanism may explain why many distinct types of RNA processing occur through compartmentalization of regulatory ncRNAs and proteins near their nascent RNA targets. Specifically, we show that each of these RNA processing hubs consists of a high concentration of nascent RNA near its transcriptional locus and enrichment of diffusible trans-associating ncRNAs - known to bind to the encoded nascent RNA - within the spatial compartment. In this way, these nuclear compartments contain high concentrations of regulatory RNAs and proteins in proximity to their nascent RNA targets, which are further organized within higher-order DNA structures that come together in 3D space to form distinct processing hubs. Because the efficiency of a biochemical reaction is increased when the substrate or enzyme concentration is increased, creating a high local concentration of regulators (e.g. spliceosomes) and targets (e.g. nascent pre-mRNAs) in 3D space may increase the kinetic efficiency of such reactions, 363 and in turn increase the efficiency of co-transcriptional processing and regulation. This 364 compartmentalization mechanism can also increase the rate at which regulators identify and engage 365 targets, which may be particularly important in cases where the regulators (e.g. scaRNAs, U7) are expressed at low levels relative to their more abundant substrates (e.g. snRNAs, histone mRNAs). This spatial organization may be an important regulatory mechanism for ensuring the efficiency of cocoupled. 
370 Our results demonstrate that hundreds of nuclear ncRNAs are preferentially localized within precise 371 structures in the nucleus, suggesting that this may be an important and common function exploited by 372 additional nuclear RNAs to coordinate the spatial organization of diffusible molecules. This mechanism 373 exploits a privileged and unique role for RNA in the nucleus (relative to DNA or proteins). Specifically, the process of transcription produces many copies of an RNA, which are by definition present at high concentrations in proximity to their transcriptional loci ${ }^{18,116}$. In contrast, proteins are translated in the cytoplasm and therefore lack positional information in the nucleus, and DNA is only present at a single copy and therefore cannot achieve high local concentrations.

Central to this mechanism is the fact that ncRNAs can form high affinity interactions with both protein and RNA immediately following transcription. In this way, they can act to recruit proteins and RNAs within these high concentration spatial compartments. In contrast, mRNAs are functional when translated into protein and do not form stable interactions with regulatory molecules in the nucleus. In this way, our results suggest that any RNA that functions independently of its translated product may similarly act as a ncRNA. For example, we note that nascent pre-mRNAs may also have protein-coding independent functions and form high-affinity interactions within the nucleus that are important for spatial organization. Indeed, we find that nascent pre-mRNAs and histone pre-mRNAs can seed organization of nuclear compartments even though their processed RNAs are also translated into protein products. This role for RNA as a seed for nuclear compartments might also explain formation of other recently described nuclear compartments such as transcriptional condensates ${ }^{11,12}$, which inherently produce high levels of RNA, including enhancer-associated RNAs and pre-mRNAs ${ }^{117}$. Nonetheless, not all ncRNAs - or even all nuclear ncRNAs - act to form compartments around their loci since nuclear ncRNAs can also localize within other regions in the nucleus (e.g. Malat1, scaRNAs, snoRNAs, and snRNAs). Future work will be needed to understand why some specific nuclear RNAs are constrained to local spatial compartments, while others diffuse throughout the nucleus.

This unique role for ncRNAs in the nucleus may explain why certain biological processes utilize ncRNA regulators rather than proteins or DNA. For example, coordinated regulation of multiple genomic DNA targets would be ideally controlled through the expression of a single ncRNA that could localize and recruit regulatory proteins to all of these targets simultaneously. Indeed, many multi-gene regulatory programs, such as $\mathrm{X}$ chromosome inactivation and imprinted gene silencing, utilize ncRNAs as regulators (e.g. Xist, Kcqn1ot1, and Airn). In this way, ncRNAs can increase both the efficiency and specificity of 
400 gene regulation by enabling control of multiple target genes through the expression of a single regulatory 401 RNA from its genomic locus. This strategy may also be advantageous even when modulating a single 402 gene because establishment of an RNA compartment can recruit effector proteins simultaneously to many 403 genomic regions that are far away in linear distance but proximal in 3D space - including promoters and 404 multiple enhancers - to enable higher concentration and more potent gene regulation. As an example, we 405 observe high concentration of the Pvt1 lncRNA over the Myc gene and all of its known enhancer elements. 406 This coordinated gene regulation model may extend to many of the hundreds of ncRNAs that we identified 407 to be localized within discrete spatial compartments in the nucleus.

408 Taken together, these results provide a global picture of how spatial enrichment of ncRNAs in the nucleus 409 can seed formation of compartments that coordinate the efficiency and specificity of a wide range of 410 essential nuclear functions, including RNA processing, heterochromatin organization, and gene regulation 411 (Supplemental Figure 8). While we focused our analysis on ncRNAs in this work, we note that RD412 SPRITE can also be applied to measure how gene expression relates to genome organization because it 413 can detect the arrangement of nascent pre-mRNAs relative other RNAs (e.g. enhancer RNAs, pre414 mRNAs) and 3D DNA structure. Beyond the nucleus, we anticipate that RD-SPRITE will also provide a 415 powerful method to study the molecular organization, function, and mechanisms of RNA compartments 416 and granules throughout the cell. 


\section{ACKNOWLEDGEMENTS}

419 We thank Elizabeth Soehalim for help in adapting the SPRITE method; Sam Kim, Vickie Trinh, and 420 Jasmine Thai for help with generating and validating cell lines and cell culture; Patrick McDonel for 421 discussions and advice on the RD-SPRITE method and helpful comments on the manuscript; Andres 422 Collazo for microscopy help; John Rinn, Drew Honson, Mackenzie Strehle, and Drew Perez for comments 423 on the manuscript and helpful suggestions; Aaron Lin for sequencing help and advice; Shawna Hiley for 424 editing; Inna-Marie Strazhnik and Sigrid Knemeyer for illustrations. S.A.Q. received support from the 425 HHMI Gilliam Fellowship and NSF GRFP Fellowship. We thank Alexander Shishkin, Ward G. Walkup 426 IV, Parham Peyda, and Vickie Trinh for help generating the Halo-tagged FL-SHARP vector. P.B. received 427 support from NIH 5 T32 GM 7616-40, NIH NRSA CA247447, and the UCLA-Caltech Medical Scientist 428 Training Program. N.O. is supported by the American Cancer Society Postdoctoral Fellowship (PF-17429 240-01). J.W.J. is supported by a BBE post-doctoral fellowship from Caltech. A.K.B. was funded by 430 NHLBI F30-HL136080 and the USC MD/PhD Program. Imaging was performed in the Biological 431 Imaging Facility, with the support of the Caltech Beckman Institute and the Arnold and Mabel Beckman 432 Foundation. This work was funded by the NIH 4DN (U01 DA040612 and U01 HL130007), the NYSCF, 433 NIH Director's Early Independence Award (DP5OD012190), CZI Ben Barres Early Career Acceleration 434 Award, Sontag Foundation, Searle Scholars Program, Pew-Steward Scholars program, and funds from the 435 California Institute of Technology. M.G. is a NYSCF-Robertson Investigator.

\section{AUTHOR CONTRIBUTIONS}

S.A.Q. conceived of this project with M.G., led the development and optimization of the RD-SPRITE method, performed experiments, analyzed and interpreted data, generated figures, oversaw all aspects of the project, and wrote the paper. P.B. developed and optimized the RD-SPRITE protocol, performed SPRITE experiments, analyzed and interpreted data, contributed to data visualization, figure presentation, model schematics/illustrations, and wrote the paper. N.O. led the effort to analyze and interpret data, wrote software, created new methods for data analysis and visualization, performed analysis and visualization on the data and contributed major findings and results, created main and supplemental figures, and contributed to the initial draft of paper, model schematics/illustrations, and reviewed and edited the manuscript. J.W.J. designed, performed, acquired, and analyzed all the RNA-FISH, DNA-FISH, IF, 
447 IF/RNA-FISH experiments and made all imaging figures; performed all LNA-related experiments and 448 generated the figures and results; performed Flavopiridol treatments and analysis; contributed to the 449 writing of the centromeric RNA hub section, model schematics/illustrations, and provided comments and 450 edits on the entire manuscript. A.K.B. performed all Kcnq1ot1 biochemical and functional experiments, 451 including CRISPRi knockdowns, TSA treatments, and functional characterizations; worked with A.C. to 452 develop and characterize the inducible Kcnqlotl cell line and to generate homozygous deletions of the 453 SHARP Binding Site within Kcnq1ot1; worked with MRB to purify SHARP and map it to Kcnq1ot1. P.C. 454 led the effort on the data processing and curation, writing scripts and constructing pipelines that enabled 455 data interpretation; was responsible for gene, repeat, and allele annotation as well as validation and 456 producing several QC metrics; contributed to experimental optimization of the RNA-DNA SPRITE 457 protocol. M.R.B. developed the engineered SHARP lines for CLAP and methods for purification of 458 SHARP; worked with A.K.B. to perform SHARP purifications for Kenq1 ot1 binding; advised and helped 459 to develop and optimize the RNA molecular biology of the RD-SPRITE method in this project. A.C. 460 developed all engineered cell lines used in this study, including the doxycycline inducible Xist cell lines, 461 Kcnq1 ot 1 lines, SHARP binding site deletions, and dCas9 cell lines. Y.M. performed all live-cell 3D-SIM 462 imaging and analysis of FL-SHARP and $\triangle$ RRM-SHARP localization. K.P. provided guidance and support 463 on imaging, analysis, ideas, and discussions on the paper. M.G. conceived of this project with S.A.Q. and 464 oversaw all experiments and analysis; performed computational analysis and generated scripts for 465 analyzing the RD-SPRITE data; wrote the paper with S.A.Q. and P.B.

\section{DECLARATION OF INTERESTS}

A provisional patent has been filed for the SPRITE method. 


\section{MAIN FIGURE LEGENDS}

470 Figure 1: RD-SPRITE generates maps of higher-order RNA and DNA contacts throughout the cell.

471 (A) Schematic of the RD-SPRITE protocol. Crosslinked cells are fragmented into smaller crosslinked 472 complexes (e.g. A, B). RNA and DNA are each tagged with a DNA-specific or RNA-specific adaptor 473 sequence (pink). The sample is processed through multiple rounds of split-and-pool barcoding ( $n$ times), 474 where tag sequences are concatemerized during each round. A series of tags is referred to as a SPRITE 475 barcode. RNA and DNA are sequenced, and barcodes are matched to generate SPRITE clusters to identify 476 all interacting molecules. (B) RNA-DNA interactions of various non-coding RNAs. Xist (burgundy) 477 unweighted contacts across the genome in female ES cells where Xist is induced exclusively on the 129 478 allele (Xi), but not the Castaneous allele (Xa). U1 spliceosomal RNA (red) and Malat1 lncRNA (grey) 479 weighted contacts across the genome occur at highly transcribed RNA Pol II (ENCODE) genomic regions 480 (black). Insets show zoom-ins of Xist (right) and U1/Malat1 along with genomic localization of RNA Pol 481 II from ENCODE (middle and left). Masked regions on chromosome X plotted in gray.

Figure 2: Non-coding RNAs form hubs containing RNAs of shared functional roles in different cellular locations. (A) A heatmap showing the number of unweighted RNA-RNA contacts between different classes of RNAs. Columns: translation-associated RNAs (18S, 28S, 5.8S, and 5S) and splicingassociated RNAs (U1, U2, U4, U5, U6). Rows: Introns and exons of individual mRNAs. Orange represents high contact frequency and blue represents low contact frequency. (B) A heatmap showing RNA-RNA unweighted contact frequencies for several classes of RNAs. Orange represents high contact

frequency and blue represents low contact frequency. Groups of RNAs that have high contact frequencies with each other, but not other RNAs, are referred to as RNA hubs.

Figure 3: Nucleolar and spliceosomal RNAs form genome-wide interaction hubs. (A) Genome-wide weighted RNA-DNA contacts (1Mb resolution) for several RNAs within the nucleolar (blue) and spliceosomal (red) hubs. RNA Pol II occupancy from ENCODE is shown along with gene density across the genome. Chromosomes that contain genes for ribosomal RNA, which are located at the centromere proximal regions of each chromosome, are demarcated in blue (chr. 12, 15, 16, 18, and 19). Blue and red horizontal heatmaps represent RNA-DNA interactions of the 45S (3'end) pre-rRNA and U1 snRNA (1Mb resolution). (B) SPRITE can measure the 3D organization of DNA occurring within RNA hubs. Multiway RNA-DNA clusters can be used to measure DNA-DNA contacts (illustrative heatmap) occurring 
specifically at DNA loci interacting with a given RNA (RNA1 clusters, red contacts) or across all SPRITE clusters (All clusters, gray contacts), as shown in the upper half and lower half of the diagonal, respectively. (C) Overlay of RNA-DNA contact frequencies on chromosome 11 is shown for various RNAs within the nucleolar hub. (D) Weighted DNA-DNA contacts within SPRITE clusters containing nucleolar hub RNAs (e.g 45S pre-rRNAs, snoRNAs, Rmrp). Long range, higher-order inter-chromosomal nucleolar interactions are shown between chromosomes 12 and 19 and chromosomes 15 and 16 for nucleolar hub RNA-containing clusters. (E) Schematic of our observations showing nascent pre-rRNAs in spatial proximity to their loci and snoRNAs and Rmrp binding to these RNAs that are spatially concentrated within this compartment. (F) Overlay of RNA-DNA contact frequencies on chromosome 11 is shown for the spliceosomal hub examples above. (G) Weighted DNA-DNA contacts within SPRITE clusters containing spliceosomal hub RNAs (e.g. U1, U2, Malat1, 7SK). Long range, higher-order interchromosomal spliceosomal hub interactions are shown between regions on chromosome 4 and chromosomes 8 and 11 (examples that have high Pol II occupancy) for all spliceosomal hub RNAcontaining clusters. (H) Schematic of our observation showing nascent pre-mRNAs in spatial proximity to their transcriptional loci and snRNAs (e.g. U1 and U2 shown) that bind to pre-mRNAs that, along with Malat1 and 7SK, are spatially concentrated within this compartment around transcribed genomic DNA.

Figure 4: Non-coding RNAs involved in snRNA and histone mRNA biogenesis are organized around snRNA and histone gene clusters. (A) Weighted RNA-DNA contacts for scaRNA2, scaRNA5 (Gm25395), and scaRNA17 (green) and U7 RNA and histone pre-mRNAs (teal) are plotted across the genome. Insets (bottom) show zoom-ins on specific regions. Lines (top) show genomic locations of each RNA plotted and gene cluster of interest. (B) Weighted DNA-DNA contacts within SPRITE clusters containing scaRNAs (upper diagonal) along with all weighted DNA-DNA contacts within all SPRITE clusters (lower diagonal) are shown across a region of chromosome 11. The locations of scaRNA occupancy is shown along the top and side axes and demarcated by a solid red box. (C) Weighted DNADNA contacts within SPRITE clusters containing the U7 RNA (upper diagonal) along with all weighted DNA-DNA contacts within all SPRITE clusters (lower diagonal) are shown across a region of chromosome 13. U7 and histone occupancy is shown along the top and side axis and demarcated with a teal box. (D) RNA FISH of scaRNAs (pooled scaRNA2 and scaRNA17 probes) along with IF of a known histone locus body (HLB) marker (NPAT) show proximity of the two compartments. Scalebar is $10 \mu \mathrm{m}$. 
snRNA gene clusters. (F) Schematic showing U7 RNA spatially concentrated near the transcribed genomic loci of target histone mRNA gene loci.

\section{Figure 5: Satellite-derived ncRNAs organize HP1 localization at inter-chromosomal hubs. (A)} Unweighted RNA-DNA contact frequencies of major (dark purple) and minor (light purple) satellitederived ncRNAs on DNA. (B) Aggregate unweighted contact frequencies of major and minor satellitederived RNAs on DNA across all chromosomes. (C) Weighted inter-chromosomal DNA-DNA contacts within SPRITE clusters containing satellite-derived RNAs. (D) DNA FISH on major (yellow) and minor (red) satellite DNA shows multiple centromeres organize around distinct chromocenter structures within a nucleus (DAPI). Dashed lines demarcate two chromocenter structures shown on the right. Specifically, we observe multiple individual centromeres (visualized by minor satellite DNA) and the larger pericentromeric regions (visualized by major satellite DNA) organized at the focal DAPI-dense chromocenter structures. Scalebar is $10 \mu \mathrm{m}$. (E) LNA-mediated knockdown of major and minor satellite-derived RNA (referred to as MajSat and MinSat RNA, respectively). (Left) Control LNA knockdown and HP1 $\beta$ immunofluorescence. (Middle) Disruption of HP1 $\beta$ foci with LNA knockdown of MajSat RNA. (Right) Disruption of HP1 $\beta$ foci with LNA knockdown of MinSat RNA. Scalebar is $10 \mu \mathrm{m}$. (F) Quantification of the mean number of HP1 foci per cell in (E). HP1 $\beta$ foci above a given intensity threshold were quantified (see Methods). Violin plot provided in Supplemental Figure 5E. Control: $n=64$ cells, MinSat: $n=80$ cells, MajSat: $n=65$ cells. Error bars represent standard error. (G) Schematic showing satellite RNAs (red gradient) spatially concentrated near the pericentromeric and centromeric DNA around a heterochromatic chromocenter structure (center), which is highly enriched with HP1 protein.

Figure 6: Most IncRNAs localize at genomic targets in 3D proximity to their transcriptional loci. (A) Chromatin enrichment score for mRNAs (black) and lncRNAs (gray). Values greater than 0 represent RNAs enriched on chromatin versus values less than 0 represent RNAs depleted on chromatin. Chromatin enrichment scores for all classes of RNAs are provided in Supplemental Figure 6A-B. (B) Unweighted RNA-DNA localization maps across the genome for selected chromatin enriched (black) and chromatin depleted (red) lncRNA examples. Chromatin enrichment scores (right) for each lncRNA are listed. Red lines (bottom) show genomic locations of each RNA plotted. (C) Unweighted genome-wide RNA-DNA localization map of 642 lncRNAs (rows) ordered by the genomic position of their transcriptional loci. (D) A 3D space filling nuclear structure model of the selected lncRNAs shown in (B). (E) A 3D space filling nuclear structure model based of 543 lncRNAs that display at least 50-fold enrichment in the nucleus. 
558 Each sphere corresponds to a $1 \mathrm{Mb}$ region or larger where each lncRNA is enriched. (F) Analysis of global 559 run on sequencing (GRO-seq) data from Jonkers et. al. (2014) ${ }^{99}$ comparing the fold change in RNA levels 560 (TPMs) between untreated and 50 minutes of flavopiridol (FVP) in mouse ES cells. Changes in introns 561 (blue), mRNAs (black), and lncRNAs (gray) are shown. Box and whiskers plot represents median, box extends from 25 th to 75 th percentiles, and whiskers are drawn from the 10th to 90th percentiles. (G) RNA FISH for selected introns (Gtdc1, Mbd5, Atrx), mRNA exons (Atrx), and lncRNAs (Pvt1, Dleu2) treated for 1 hour with DMSO or FVP are shown. Scalebar is $10 \mu \mathrm{m}$.

565 Figure 7: Kenq1ot1 seeds an RNA-mediated compartment over its imprinted target genes and binds the SHARP protein to repress gene expression. (A) Diagram of the functional domains in full length (FL) SHARP (also referred to as Spen) protein. SHARP contains four RNA recognition motif (RRM, blue) domains and one Spen paralogue and orthologue C-terminal (SPOC, orange) domain. A version of SHARP lacking its RNA binding motifs $(\triangle \mathrm{R} R \mathrm{R})$ was generated by deletion of the first 591 amino acids of SHARP. (B) Top 3D-SIM $125 \mathrm{~nm}$ optical sections of FL-SHARP (left) and $\triangle$ RRM-SHARP (right) and z-projections (bottom) Halo-tagged FL- and $\triangle$ RRM- SHARPJF646. FL-SHARP localizes in foci throughout the nucleus (zoom in panels 1-2), while $\triangle$ RRM-SHARP leads to diffusive localization. Bar: $5 \mu \mathrm{m}$, insets: $0.5 \mu \mathrm{m}$ (magnifications). Intensities are depicted in 16-color grading from black (minimum) to white (maximum). (C) SHARP (also referred to as Spen) protein binding on the entire 84,000 nucleotide Kcnq1 lot1 lncRNA (top), and a zoom in on the first 5,000 nucleotides of the lncRNA (bottom). We define a region called the SHARP binding site (SBS) shown as a black box. (D) Weighted DNA-DNA contacts within SPRITE clusters containing the Kcnq1ot1 RNA. Dashed line indicates the location of the Kcnq1ot1-enriched spatial compartment. (Zoom out) Genomic locations in this domain of the Kenq1ot1 gene (burgundy) and imprinted target genes Kcnq1, Slc22a18, Cdkn1c, and Phlda2 (black) and nonimprinted neighboring genes Nap114 and Cars (gray). (E) Changes in mean gene expression upon CRISPR inhibition (CRISPRi) of the Kcnq1ot1 lncRNA. Genes contained within the Kcnq1ot1-associated domain (e.g. Cdkn1c, Phlda2, Slc22a18) are shown in black and genes outside the domain (e.g. Cars, Nap114) are shown in gray. Error bars represent standard deviation. (F) Changes in mean gene expression upon doxycycline mediated induction (+Dox) of Kcnq1ot1 relative to cells with no doxycycline (-Dox). Genes contained within the Kcnq1ot1-associated domain (e.g. Cdkn1c, Phlda2, Slc22a18) are shown in black and genes outside the domain (e.g. Cars, Nap114) are shown in gray. Error bars represent standard deviation. (G) Homozygous deletion of the SHARP binding site $(\Delta \mathrm{SBS})$ in two different clones results in up-regulation of the genes within the Kcnq1ot1-domain (e.g. Cdkn1c, Phlda2), but does not impact the 
neighboring genes outside this domain (e.g. Cars, Nap114). (H) Schematic of our results for the Kcnq1ot1-

590 mediated compartment. Kcnqlot1 lncRNA seeds the formation of an RNA-mediated compartment in 591 spatial proximity to its transcriptional locus, and then binds and recruits the SHARP protein into this 592 compartment to silence its imprinted target genes.

593 Figure 8: A model for the mechanism by which ncRNAs drive the formation of nuclear 594 compartments. Upon transcription, mRNAs are exported to the cytoplasm (for translation to proteins) 595 while ncRNAs are retained in the nucleus. The process of ncRNA transcription creates a concentration 596 gradient of ncRNA transcript with the highest concentrations near its transcriptional locus (SEED, left 597 panel). Because these RNAs are functional immediately upon transcription and can bind with high affinity 598 to diffusible RNAs and proteins (BIND, middle panel), they can act to change the dynamic equilibrium 599 of these proteins to concentrate them in a spatial compartment (RECRUIT, right panel). In this way, 600 ncRNAs can drive the organization of regulatory and functional nuclear compartments containing RNA, 601 DNA and proteins. 


\section{SUPPLEMENTAL FIGURE LEGENDS}

603 Supplemental Figure 1: RD-SPRITE accurately measures RNA and DNA contacts. (A) Schematic 604 of DNA and RNA tagging with sequence-specific tags to identify DNA- and RNA-specific reads through 605 sequencing. DNA and RNA are each tagged with sequence-specific tags, namely "DNA Phosphate 606 Modified" (DPM) tag and "RNA Phosphate Modified" (RPM) tags using T4 DNA and RNA Ligase, 607 respectively. DNA is double stranded and therefore DPM will be read from both strands, while RNA is 608 single stranded and therefore RPM will be read only from 1 strand. Additionally, the RPM and DPM tags 609 have identical dsDNA sticky ends that enable subsequent split-pool barcoding with the same SPRITE 610 tags. (B) The percentage of reads aligning to each DNA strand based on their DPM (DNA reads) or RPM 611 (RNA reads) tags is shown across 144 independently amplified and sequenced SPRITE libraries from two 612 SPRITE experiments. (C) Percentage of reads in SPRITE clusters of different sizes, stratified into 613 categories of clusters containing 1, 2-10, 11-100, 101-1000, and 1001+ reads per cluster. Distributions 614 shown for all clusters (left) and paired clusters (2+ reads per cluster) (right). (D) Percentage of DNA reads 615 within each chromosome contained within SPRITE clusters containing the Xist RNA (black) compared 616 to all SPRITE clusters (gray). (E) The aggregate unweighted contact frequency of the Telomerase 617 associated RNA Component (Terc) across all chromosomes is shown.

618 Supplemental Figure 2: RNA localization on DNA and within the nucleus for RNAs within each 619 RNA hub. (A) Weighted genomic DNA localization heatmap of each individual RNA. RNAs are 620 organized by their RNA hub occupancy (shown in Figure 2A). Contacts are normalized from 0 to 1 to 621 account for expression levels of each RNA. (B) Pearson correlation of RNA-DNA unweighted contact 622 frequencies across the genome for individual RNAs within the nuclear hubs (nucleolar, centromeric, 623 spliceosomal, and scaRNA hubs). Red represents high correlation and blue represents low correlation. (C) 624 RNA FISH of various non-coding RNAs within the same hub in the nucleus. Spliceosomal hub (top): 625 Malat1 lncRNA and 7SK RNA and (bottom): U6 and U1 spliceosomal RNAs. Nucleolar hub (top): snora26 snoRNA and 45S pre-rRNA ITS2 and (bottom): RNase MRP (Rmrp) and 45S pre-rRNA ITS1.

627 Each panel is shown individually (left and middle) and overlaid (right). Dashed lines demarcate the nuclear 628 boundary identified with DAPI. Scalebar is $10 \mu \mathrm{m}$. (D) RNA FISH (left) along with nucleolin 629 immunofluorescence (middle) and DAPI (right) of specific ncRNAs. 7SK RNA (top), ITS1 regions of 630 45S pre-rRNA (middle) and tRNAs (bottom). tRNAs are visualized using pooled RNA FISH probes (see 631 Methods). Scalebar is $10 \mu \mathrm{m}$. 


\section{Supplemental Figure 3: Nucleolar and spliceosomal hubs show higher-order interactions around}

633 loci of rRNA and mRNA genes, respectively. (A) Genome-wide localization of each individual 634 snoRNA. Blue track shows 45S pre-rRNA localization on DNA. Chromosomes containing ribosomal 635 DNA genes (chromosomes 12, 15, 16, 18, 19) are denoted in blue. (B) Weighted DNA-DNA contact heatmap shown for SPRITE clusters containing any of the RNAs within the nucleolar hub (left), both snoRNAs and 45S pre-rRNA (middle), and snoRNAs, 45S, and 5S (right) simultaneously. (C) $1 \mathrm{Mb}$ Enrichment of several spliceosomal hub RNA-DNA interactions (U1 snRNA, U2 snRNAs, 7SK RNA, and Malat1 lncRNA) compared to enrichment of Pol II ChIP-seq signal (ENCODE) genome wide. Pearson correlation provided for each set of RNA-DNA interactions and Pol II signal comparisons. (D) DNADNA contact heatmap shown for SPRITE clusters containing any of the RNAs contained within the spliceosomal hub (left) or containing 2 or more distinct spliceosomal hub RNAs simultaneously (right).

Supplemental Figure 4: Spatial relationship between snRNA biogenesis hub and histone locus

bodies. (A) Immunofluorescence imaging of classical Cajal Body (Coilin) and nuclear gem (SMN) markers in mouse ES cells and HEK293T cells. Top: Mouse ES cells do not contain visible Coilin foci for any of the three anti-Coilin antibodies tested. Bottom: HEK293T cells show visible Coilin foci. SMN foci, which are markers for nuclear Gemini of Cajal bodies ("gems") are present in both mouse ES cells and HEK293T cells. (B) Z-section of mouse ES cell co-stained for SMN protein and scaRNAs (pooled scaRNA2 and scaRNA17 probes) within the nucleus (DAPI). Inset shows an example of scaRNA localization near SMN foci (arrow). (C) Z-section of mouse ES cell with RNA FISH staining for U7 and scaRNAs (pooled scaRNA2 and scaRNA17 probes) within the nucleus (DAPI). Inset shows an example of scaRNA localization near U7 (arrow). (D) RNA-RNA contact frequency between scaRNA2 and all RNAs. Top hits include annotated scaRNAs and identify two previously unannotated scaRNAs (see Supplemental Methods). (E) Weighted DNA-DNA contacts for all SPRITE clusters (top) and for SPRITE clusters containing scaRNAs (bottom) occurring within a region on chromosome 11 with snRNA gene clusters. scaRNA occupancy is demarcated with solid red boxes. (F) Weighted DNA-DNA contacts for all SPRITE clusters (top) and for SPRITE clusters containing the U7 ncRNA (bottom) occurring within a region on chromosome 13 containing the two Hist1 gene clusters. U7 and Hist1 RNA occupancy is demarcated with teal boxes. (G) Weighted DNA-DNA contacts shown for SPRITE clusters containing both scaRNAs and snRNAs simultaneously. (H) Weighted DNA-DNA contacts for SPRITE clusters containing the scaRNAs on chromosome 13. 


\section{Supplemental Figure 5: Satellite-derived ncRNAs mediate higher-order heterochromatin} organization at centromeric clusters. (A) Weighted DNA-DNA contact matrices constructed from SPRITE clusters containing minor and major satellite RNAs. (B) 3D projections of either MajSat RNA FISH (top) or MinSat RNA FISH (bottom). DAPI in blue. Dashed lines and corresponding inset boxes zoom in on a single DAPI-dense chromocenter structure. (C) Quantification of mean LNA knockdown for minor satellite RNA (2 primer sets) compared to control LNA. Error bars represent standard deviation. (D) Quantification of LNA knockdown for major satellite RNA (2 primer sets) compared to control LNA. Error bars represent standard deviation. (E) Quantification of number of HP1 foci shown in Figure 5E (as a violin plot). Control: $n=64$ cells, MinSat: $n=80$ cells, MajSat: $n=65$ cells. (F) Imaging of DNA FISH in control (left) or LNA knockdown of MajSat (middle) and MinSat (right) RNA. DNA-FISH of MajSat shown as a color gradient indicating DNA FISH signal intensity (top) and with DAPI (bottom). See Supplemental Note 4 for detailed description of phenotypes. Scale bar is $10 \mu \mathrm{m}$.

\section{Supplemental Figure 6: Many IncRNAs localize within 3D proximity to their transcriptional loci in}

the nucleus. (A) Schematic illustration of our chromatin enrichment score which computes the frequency of an RNA interaction with chromatin (top inset) compared to the frequency of interactions without chromatin, such as with rRNA, tRNA, and mRNA interactions in the cytoplasm (bottom inset). (B) Chromatin enrichment score for multiple classes of RNAs. tRNAs, rRNAs, and exons are predominantly depleted on chromatin (enrichment score $<0$ ) versus other classes of RNAs, including introns, scaRNAs, lncRNAs, are enriched on chromatin (enrichment score $>0$ ). (C) RNA FISH localization patterns of multiple lncRNAs (Xist, Malat1, Tsix, Kcnq1ot1, Pvt1, and Dleu2 lncRNAs) in the nucleus (DAPI). (D) Normalized RNA-DNA interactions for several lncRNAs (blue) and mRNAs (red). Each RNA locus is demarcated at the bottom. (E) Chromatin enrichment scores (x-axis) versus ribosomal RNA enrichment scores (y-axis) for exons (red), introns (blue), and lncRNAs (purple). (F) RNA FISH for 4 mRNA introns (Ehmt4, Nup188, Abi1, Gtdc2) and 4 lncRNAs (Kcnq1ot1, Tsix, Pvt1, Dleu2) treated for 1 hour with DMSO (top) or FVP (bottom). As a control, we co-stained lncRNAs (white) and introns (red) within the same cell.

Supplemental Figure 7: IncRNAs regulate target gene expression precisely within their localization 
692 A (TSA) (right). DMSO: Regulated genes show robust repression while genes not within the imprinted 693 TAD show no difference in expression upon induction of Kcnq1ot1. TSA: Regulated genes show loss of 694 repression and exhibit comparable gene expression changes with genes not within the imprinted TAD. 695 Error bars represent standard deviation. (C) RNA FISH performed with two distinct probes targeting the 696 Kcnq1 lot1 lncRNA - 1 probe set was designed against the 3' end of the RNA and the other designed 697 against the 5' end of the RNA. FISH was performed in cells in the absence of doxycycline (left) and in 698 the presence of doxycycline (right). (D) Weighted DNA-DNA interaction matrix for Pvt1 RNA-containing 699 SPRITE clusters showing Pvt1 lncRNA localization on DNA in a region occupied by Pvt1 and Myc genes.

700 (E) Weighted DNA-DNA interaction matrix for Airn RNA-containing SPRITE clusters showing Airn 701 lncRNA localization on DNA in a region confined to the genes Airn is known to regulate ${ }^{118}$. (F) Weighted 702 DNA-DNA interaction matrix for Chaserr RNA-containing SPRITE clusters. Chaserr RNA is confined to 703 a TAD containing the Chaserr gene and its known regulatory target, Chd2. (G) Top: DNA-DNA contacts 704 within SPRITE clusters containing each lncRNA (left: Kcnq1ot1, middle: Airn, right: Pvt1) and bottom: 705 DNA-DNA contacts across all SPRITE clusters within the same regions.

706 Supplemental Figure 8: A widespread role for ncRNAs in shaping compartments throughout the 707 nucleus that are associated with various nuclear functions. A schematic of the localization of the 708 different nuclear compartments within the nucleus and the molecular components contained within them. 709 In each of these cases, an RNA seeds organization by achieving high concentration in spatial proximity to 710 its transcriptional locus. This leads to the formation of nuclear compartments associated with RNA 711 processing, heterochromatin assembly, and gene regulation.

712 Supplemental Video 1: Full length SHARP localizes in discrete diffraction-limited foci. Live-cell 3D713 SIM of Halo-tagged FL-SHARP JF646 captured for $\sim 2$ minutes reveals distinct and persistent SPEN foci 714 throughout the nucleus.

715 Supplemental Video 2: Deletion of the RNA recognition motifs of SHARP leads to diffusive 716 localization. Live-cell 3D-SIM of $\triangle$ RRM-SHARP JF646 captured for $\sim 2$ minutes exhibits a diffusive 717 localization pattern and no observable foci in the nucleus. 


\section{SUPPLEMENTAL NOTES}

720 Supplemental Note 1: RD-SPRITE improves efficiency of RNA tagging. Although our previous version 721 of SPRITE could map both RNA and DNA, it was limited primarily to detecting highly abundant RNA 722 species (e.g. 45S pre-rRNA). In RD-SPRITE, we have improved detection of lower abundance RNAs by 723 increasing yield through the following adaptations. (i) We increased the RNA ligation efficiency by 724 utilizing a higher concentration of RPM, corresponding to 2000 molar excess during RNA ligation. (ii) 725 Adaptor dimers that are formed through residual purification on our magnetic beads lead to reduced 726 efficiency because they preferentially amplify and preclude amplification of tagged RNAs. To reduce the 727 number of adaptor dimers in library generation, we introduced an exonuclease digestion of excess reverse 728 transcription (RT) primer that dramatically reduces the presence of the RT primer. (iii) Reverse 729 transcription is used to add the barcode to the RNA molecule, yet when RT is performed on crosslinked 730 material it will not efficiently reverse transcribe the entire RNA (because crosslinked proteins will act to 731 sterically preclude RT). To address this, we performed a short RT in crosslinked samples followed by a 732 second RT reaction after reverse crosslinking to copy the remainder of the RNA fragment. (iv) Because 733 cDNA is single stranded, we need to ligate a second adaptor to enable PCR amplification. The efficiency 734 of this reaction is critical for ensuring that we detect each RNA molecule. We significantly improved 735 cDNA ligation efficiency by introducing a modified "splint" ligation. Specifically, a double stranded 736 "splint" adaptor containing the Read1 Illumina priming region and a random 6mer overhang is ligated to 737 the 3'end of the cDNA at high efficiency by performing a double stranded DNA ligation. This process is 738 more efficient than the single stranded DNA-DNA ligation previously utilized ${ }^{54}$. (v) Finally, we found 739 that nucleic acid purification performed after reverse crosslinking leads to major loss of complexity 740 because we lose a percentage of the unique molecules during each cleanup. In the initial RNA-DNA 741 SPRITE protocol there were several column (or bead) purifications utilized to remove enzymes and enable 742 the next enzymatic reaction. We reduced these cleanups by introducing biotin modifications into the DPM 743 and RPM adaptors that enable binding to streptavidin beads and for all subsequent molecular biology steps 744 to occur on the same beads. Together, these improvements enabled a dramatic improvement of our overall 745 RNA recovery and enables generation of high complexity RNA/DNA structure maps.

746 Supplemental Note 2: The snRNA biogenesis hub may be similar to the Cajal body. We note that the 747 snRNA biogenesis hub may be similar to Cajal bodies, which have been noted to contain snRNA genes 748 and scaRNAs ${ }^{82,84,86,119,120}$. However, Cajal bodies are traditionally defined by the presence of Coilin foci 
in the nucleus ${ }^{80,84,121}$ and based on this definition, our mES cells do not contain visible Cajal bodies with all three antibodies tested (Supplemental Figure S4A). Despite the absence of traditionally defined Cajal bodies, our data suggest that snRNA biogenesis hubs do indeed exist and form around snRNA gene loci, even in the absence of observable Coilin foci. Our data suggest that scaRNA localization more accurately defines snRNA processing bodies relative to Coilin. Consistent with this idea, scaRNAs have a clearly defined functional role in snRNA biogenesis whereas Coilin is dispensable for snRNA biogenesis ${ }^{86}$. It is also possible that these snRNA processing bodies are distinct from Cajal bodies, which may represent a different nuclear structure. For example, these might represent nuclear gems ${ }^{122}$, which contain SMN protein, or "residual bodies," which are Coilin negative ${ }^{123,124}$. We note that we observe SMN foci in our mES cells and that some, but not all, scaRNAs colocalize with SMN protein in the nucleus (Supplemental Figure S4A-B).

Supplemental Note 3: RD-SPRITE measures the frequency at which RNAs are contacting chromatin. Although data from previous methods have reported that both lncRNAs and mRNAs are similarly enriched on chromatin at their transcriptional loci, we observed a striking difference in chromatin localization between these classes of RNA. The major reason for this is because RD-SPRITE measures RNA localization within all compartments of the cell, including in the nucleus and cytoplasm. Accordingly, we can compute a chromatin enrichment score, which we define as the frequency at which a given RNA is localized on chromatin (Supplemental Figure 6A-B). Other RNA-DNA mapping methods such as hybridization (e.g. RAP, ChIRP) or proximity-ligation (e.g. GRID-Seq, Margi) methods exclusively measure RNA when they are present on chromatin and therefore cannot measure this differential localization frequency.

\section{Supplemental Note 4: Depletion of satellite-derived ncRNAs leads to altered chromocenter structure.}

Chromocenter regions display altered structure $48 \mathrm{~h}$ and $72 \mathrm{~h}$ post MajSat and MinSat LNA transfection in comparison to control. All 3 conditions (MajSat, MinSat, Control) were visualized by DNA-FISH (chromocenters clustering depicted in gradient of fluorescent intensity from MajSat targeting probes) and DAPI staining (chromocenters depicted as DAPI rich regions). The observed phenotypes include: (i) larger pericentromeric chromatin foci and clusters in comparison to control samples; (ii) smaller pericentromeric chromatin clusters and foci that seem to be less condensed (rod-shaped) in comparison to controls (Supplemental Figure 5F). Our results demonstrate that both MajSat and MinSat RNAs are required for HP1 $\beta$ recruitment to chromocenter regions. 


\section{MATERIALS AND METHODS}

\section{Lead Contact}

781 Further information and requests for resources and reagents should be directed to and will be fulfilled by 782 the Lead Contact, Mitchell Guttman (mguttman@caltech.edu).

\section{Materials Availability}

785 This study did not generate new unique reagents.

\section{Data and Code Availability}

788 The analysis pipeline used in this study is available at https://github.com/GuttmanLab/sprite2.0-pipeline. 789 Datasets generated during this study will be available on GEO.

\section{Cell line generation, cell culture, and drug treatments}

Cell lines used in this study. We used the following cell lines in this study: (i) Female ES cells (pSM44 793 ES cell line) derived from a $129 \times$ castaneous F1 mouse cross. These cells express Xist from the 794 endogenous locus under control of a tetracycline-inducible promoter. The dox-inducible Xist gene is 795 present on the 129 allele, enabling allele-specific analysis of Xist induction and X chromosome silencing. 796 (ii) Female ES cells where we replaced the endogenous Kcnq1ot1 promoter with a tetracycline-inducible 797 promoter on both alleles (Kcnq1ot1-inducible ES cell line). In the absence of Doxycycline, these cells do 798 not express Kcnq1ot1 from either allele; in the presence of Doxycycline, these cells express Kcnq1ot1 799 biallelically. (iii) Female ES cells containing dCas9 fused to 4-copies of the SID transcriptional repression 800 domain integrated into a single locus in the genome (dCas9-4XSID). (iv) HEK293T, a female human 801 embryonic kidney cell line obtained from ATCC.

802 Cell culture conditions. All mouse ES cell lines were cultured in serum-free 2i/LIF medium as previously 803 described $^{54}$. HEK293T cells were cultured in complete media consisting of DMEM (GIBCO, Life Technologies) supplemented with 10\% FBS (Seradigm Premium Grade HI FBS, VWR), 1X penicillinstreptomycin (GIBCO, Life Technologies), 1X MEM non-essential amino acids (GIBCO, Life Technologies), $1 \mathrm{mM}$ sodium pyruvate (GIBCO, Life Technologies) and maintained at $37^{\circ} \mathrm{C}$ under $5 \%$ $\mathrm{CO}_{2}$. For maintenance, 800,000 cells were seeded into $10 \mathrm{~mL}$ of complete media every 3-4 days in $10 \mathrm{~cm}$ 
808 dishes. HEK293T cells were used for human-mouse mixing experiments to assess noise during the SPRITE procedure as well as for imaging Coilin foci.

Doxycycline Inducible Cell Line Development, Female ES cells (F1 2-1 line, provided by K. Plath) were CRISPR-targeted (nicking gRNA pairs TGGGCGGGAGTCTTCTGGGCAGG and GGATTCTCCCAGGCCCAGGGCGG) to integrate the Tet transactivator (M2rtTA) into the Rosa26

813 locus using R26P-M2rtTA, a gift from Rudolf Jaenisch (Addgene plasmid \#47381). This line was 814 subsequently CRISPR-targeted (nicking gRNA pairs GCTCGTTTCCCGTGGATGTG and 815 GCACGCCTTTAACTGATCCG) to replace the endogenous Xist promoter with tetracycline response elements (TRE) and a minimal CMV promoter as previously described ${ }^{46}$. The promoter replacement 817 insertion was verified by PCR amplification of the insertion locus and Sanger sequencing of the amplicon. 818 SNPs within the amplicon allowed for allele identification of the insertion, confirming that the 129 allele was targeted and induced Xist expression. We routinely confirmed the presence of two X chromosomes within these cells by checking the presence of X-linked SNPs on the 129 and castaneous alleles.

3D-SIM SHARP-Halo cell culture conditions. pSM33 cells were seeded in 4-well imaging chambers (ibidi) equipped with a high precision glass bottom and plasmids were transfected with lipofectamine 300024 hours prior to imaging according to the manufacturer's instructions. Addition of doxycycline $8 \mathrm{hrs}$ prior to imaging was performed to induce SHARP expression. 1 $\mu \mathrm{M}$ JF646 Halo ligand was introduced to the media for $30 \mathrm{~min}$, washed-off twice with PBS and exchanged with fresh media which were incubated for another $15 \mathrm{~min}$. Live-cell 3D-SIM imaging was performed at $37 \mathrm{C}$ and $5 \% \mathrm{CO}_{2}$ in media without phenol red.

Doxycycline Inducible Kcnq1ot1 lines. The endogenous promoter of Kcnq1ot1 was CRISPR-targeted (nicking gRNA pairs TCGTGGCTGCCACGTCACCA and CAGATGCTGAATAATGACTA) to insert

830 a TRE and minimal CMV promoter. Clones were screened for ablation of endogenous Kcnq1ot1 831 expression and biallelic upregulation of expression upon administration of doxycycline using RNA FISH probes targeting the 5'end and 3'end of the RNA (Supplemental Figure 7C).

833 CRISPRi: dCas9-4XSID cell line generation. A catalytically dead Cas9 (dCas9) fused to 4 copies of the 834 SID repressive domain (4XSID) expressed from an Ef1 $\alpha$ promoter was integrated into a single copy locus 835 in the genome (mm10 - chr6:86,565,487-86,565,506; gRNA sequence AATCTTAGTACTACTGCTGC) 836 using CRISPR targeting (cells hereby referred to as dCas9-4XSID). 
Doxycycline induction. Xist and Kcnq1otl expression were induced in their respective cell lines by treating cells with $2 \mu \mathrm{g} / \mathrm{ml}$ doxycycline (Sigma). Xist was induced for 24 hours prior to crosslinking and analysis. Kenq1ot1 was induced for 12-16hrs prior to RNA harvesting.

Trichostatin (TSA) treatment. For HDAC inhibitor experiments, cells were treated with either DMSO (control) or $5 \mu \mathrm{M}$ TSA (Sigma T8552-1MG) in fresh $2 \mathrm{i}$ media or $2 \mu \mathrm{g} / \mathrm{ml}$ doxycycline in standard $2 \mathrm{i}$.

Flavopiridol (FVP) Treatment. FVP transcriptional inhibition was performed by culturing cells in FVP (Sigma F3055-1MG) or DMSO at 1uM final concentration for 1 hour.

\section{RNA \& DNA-SPRITE: Simultaneous tagging of RNA and DNA interactions using SPRITE}

RD-SPRITE is an adaptation of our initial SPRITE protocol ${ }^{54}$ with significant improvements to the RNA molecular biology steps that enable generation of higher complexity RNA libraries. The approach was performed as follows:

Crosslinking, lysis, sonication, and chromatin digestion. Cells were lifted using trypsinization and were crosslinked in suspension at room temperature with $2 \mathrm{mM}$ disuccinimidyl glutarate (DSG) for 45 minutes followed by 3\% Formaldehyde for 10 minutes to preserve RNA and DNA interactions in situ. After crosslinking, the formaldehyde crosslinker was quenched with addition of $2.5 \mathrm{M}$ Glycine for final concentration of $0.5 \mathrm{M}$ for 5 minutes, cells were spun down, and resuspended in $1 \mathrm{x}$ PBS $+0.5 \%$ RNAse Free BSA (AmericanBio \#AB01243-00050) over three washes, 1x PBS + 0.5\% RNAse Free BSA was removed, and flash frozen at $-80 \mathrm{C}$ for storage. We found that RNAse Free BSA is critical to avoid RNA degradation. RNase Inhibitor (1:40, NEB Murine RNAse Inhibitor or Thermofisher Ribolock) was also added to all lysis buffers and subsequent steps to avoid RNA degradation. After lysis, cells were sonicated at 4-5W of power for 1 minute (pulses 0.7 second on, 3.3 seconds off) using the Branson Sonicator and chromatin was fragmented using DNAse digestion to obtain DNA of approximately $\sim 150 \mathrm{bp}-1 \mathrm{~kb}$ in length.

861 Estimating molarity. After DNase digestion, crosslinks were reversed on approximately $10 \mu 1$ of lysate 862 in $82 \mu \mathrm{L}$ of $1 X$ Proteinase $\mathrm{K}$ Buffer (20 mM Tris pH 7.5, $100 \mathrm{mM} \mathrm{NaCl}, 10 \mathrm{mM}$ EDTA, $10 \mathrm{mM}$ EGTA, 
864 purified using Zymo RNA Clean and Concentrate columns per the manufacturer's specifications $(>17 \mathrm{nt}$ 865 protocol) with minor adaptations, such as binding twice to the column with $2 \mathrm{X}$ volume RNA Binding 866 Buffer combined with by $1 \mathrm{X}$ volume $100 \%$ EtOH to improve yield. Molarities of the RNA and DNA 867 were calculated by measuring the RNA and DNA concentration using the Qubit Fluorometer (HS RNA 868 kit, HS dsDNA kit) and the average RNA and DNA sizes were estimated using the RNA High Sensitivity Tapestation and Agilent Bioanalyzer (High Sensitivity DNA kit).

NHS bead coupling. We used the RNA and DNA molarity estimated in the lysate to calculate the total number of RNA and DNA molecules per microliter of lysate. We coupled the lysate to NHS-activated magnetic beads (Pierce) in 1x PBS + 0.1\% SDS combined with 1:40 dilution of NEB Murine RNase Inhibitor overnight at $4^{\circ} \mathrm{C}$ as previously described ${ }^{54}$. We coupled at a ratio of 0.5 molecules per bead to reduce the probability of simultaneously coupling multiple independent complexes to the same bead, which would lead to their association during the split-pool barcoding process. Because multiple molecules of DNA and RNA can be crosslinked in a single complex, this estimate is a more conservative estimate of the number of molecules to avoid collisions on individual beads. After NHS coupling overnight, the coupling was quenched in $0.5 \mathrm{M}$ Tris $\mathrm{pH} 7.5$ and beads were washed post coupling as previously described.

Because the crosslinked complexes are immobilized on NHS magnetic beads, we can perform several enzymatic steps by adding buffers and enzymes directly to the beads and performing rapid buffer exchange between each step on a magnet. All enzymatic steps were performed with shaking at $1200 \mathrm{rpm}$ (Eppendorf Thermomixer) to avoid bead settling and aggregation. All enzymatic steps were inactivated either by adding $1 \mathrm{~mL}$ of SPRITE Wash buffer $(20 \mathrm{mM}$ Tris- $\mathrm{HCl} \mathrm{pH} 7.5,50 \mathrm{mM} \mathrm{NaCl}, 0.2 \%$ Triton-X, $0.2 \%$ NP-40, $0.2 \%$ Sodium deoxycholate) supplemented with $50 \mathrm{mM}$ EDTA and $50 \mathrm{mM}$ EGTA to the NHS beads or Modified RLT buffer (1x Buffer RLT supplied by Qiagen, 10mM Tris-HCl pH 7.5, 1mM

DNA End Repair and dA-tailing. We then repair the DNA ends to enable ligation of tags to each molecule. Specifically, we blunt end and phosphorylate the $5^{\prime}$ ends of double-stranded DNA using two enzymes. First, T4 Polynucleotide Kinase (NEB) treatment is performed at $37^{\circ} \mathrm{C}$ for 1 hour, the enzyme is quenched using $1 \mathrm{~mL}$ Modified RLT buffer, and then buffer is exchanged with two washes of $1 \mathrm{~mL}$ SPRITE Detergent Buffer to beads at room temperature. Next, the NEBNext End Repair Enzyme 
893 cocktail (containing T4 DNA Polymerase and T4 PNK) and 1x NEBNext End Repair Reaction Buffer is

894 added to beads and incubated at $20^{\circ} \mathrm{C}$ for 1 hour, and inactivated and buffer exchanged as specified

895 above. DNA was then dA-tailed using the Klenow fragment (5'-3' exo-, NEBNext dA-tailing Module) at $89637^{\circ} \mathrm{C}$ for 1 hour, and inactivated and buffer exchanged as specified above. Note, we do not use the 897 combined NEB End Repair/dA tailing modules as the temperatures in the protocol are not compatible 898 with SPRITE as the higher temperature will reverse crosslinks. To prevent degradation of RNA, each 899 enzymatic step is performed with the addition of 1:40 NEB Murine RNAse Inhibitor or Thermofisher 900 Ribolock.

901 Ligation of the DNA Phosphate Modified ("DPM") Tag. After end repair and dA-tailing of DNA, we 902 performed a pooled ligation with "DNA Phosphate Modified" (DPM) tag that contains certain 903 modifications that we found to be critical for the success of RD-SPRITE. Specifically, (i) we incorporate 904 a phosphothiorate modification into the DPM adaptor to prevent its enzymatic digestion by Exo1 in 905 subsequent RNA steps and (ii) we integrated an internal biotin modification to facilitate an on-bead 906 library preparation post reverse-crosslinking. The DPM adaptor also contains a 5'phosphorylated sticky 907 end overhang to ligate tags during split-pool barcoding. Ligation was performed as previously described 908 using Instant Sticky End Mastermix (NEB) except that all ligations were supplemented with 1:40 909 RNAse inhibitor (ThermoFisher Ribolock or NEB Murine RNase Inhibitor) to prevent RNA 910 degradation. Because T4 DNA Ligase only ligates to double-stranded DNA, the unique DPM sequence 911 enables accurate identification of DNA molecules after sequencing.

912 Ligation of the RNA Phosphate Modified ("RPM") Tag. To map RNA and DNA interactions 913 simultaneously, we ligated a RNA adaptor to RNA that contains the same 7nt 5'phosphorylated sticky 914 end overhang as the DPM adaptor to ligate tags to both RNA and DNA during split-pool barcoding. To 915 do this, we first modify the 3'end of RNA to ensure that they all have a 3'OH that is compatible for 916 ligation. Specifically, RNA overhangs are repaired with T4 Polynucleoide Kinase (NEB) with no ATP at $91737^{\circ} \mathrm{C}$ for 20 min. RNA is subsequently ligated with a "RNA Phosphate Modified" (RPM) adaptor as 918 previously described using High Concentration T4 RNA Ligase I ${ }^{125}$. Because T4 RNA Ligase 1 only 919 ligates to single-stranded RNA, the unique RPM sequence enables accurate identification of RNA and 920 DNA molecules after sequencing. After RPM ligation, RNA was converted to cDNA using Superscript 921 III at $42^{\circ} \mathrm{C}$ for 1 hour using the "RPM bottom" RT primer that contains an internal biotin to facilitate on922 bead library construction (as above) and a 5'end sticky end to ligate tags during SPRITE. Excess primer 
923 is digested with Exonuclease 1. All ligations were supplemented with 1:40 RNAse inhibitor

924 (ThermoFisher Ribolock or NEB Murine RNase Inhibitor) to prevent RNA degradation.

Split-and-pool barcoding to identify RNA and DNA interactions. The beads were then repeatedly splitand-pool ligated over four rounds with a set of "Odd," "Even" and "Terminal" tags (see SPRITE Tag 927 Design in Quinodoz et al. Cell 2018 ${ }^{54}$ ). Both DPM and RPM contain the same 7 nucleotide sticky end 928 that will ligate to all subsequent split-pool barcoding rounds. All split-pool ligation steps and reverse 929 crosslinking were performed for $45 \mathrm{~min}$ to 1 hour at $20^{\circ} \mathrm{C}$ as previously described. All ligations were 930 supplemented with 1:40 RNAse inhibitor (ThermoFisher Ribolock or NEB Murine RNase Inhibitor) to 931 prevent RNA degradation.

932 Reverse crosslinking. After multiple rounds of SPRITE split-and-pool barcoding, the tagged RNA and 933 DNA molecules are eluted from NHS beads by reverse crosslinking overnight $(\sim 12-13$ hours $)$ at $50^{\circ} \mathrm{C}$ in NLS Elution Buffer (20mM Tris-HCl pH 7.5, 10mM EDTA, 2\% N-Lauroylsarcosine, 50mM NaCl) with added $5 \mathrm{M} \mathrm{NaCl}$ to $288 \mathrm{mM} \mathrm{NaCl}$ Final combined with 5uL Proteinase K (NEB).

Post reverse-crosslinking library preparation. AEBSF (Gold Biotechnology CAS\#30827-99-7) is added to the Proteinase K (NEB Proteinase K \#P8107S; ProK) reactions to inactive the ProK prior to coupling to streptavidin beads. Biotinylated barcoded RNA and DNA are bound to streptavidin beads. To improve recovery, the supernatant is bound again to $20 \mathrm{ul}$ of streptavidin beads and combined with the first capture. Beads are washed in 1X PBS + RNase inhibitor and then resuspended in 1x First Strand buffer to prevent any melting of the RNA:cDNA hybrid. Beads were pre-incubated at 40C for 2 min to prevent any sticky barcodes from annealing. A second reverse transcription is performed by adding Superscript III (without RT primer) to extend the cDNA through the areas which were previously crosslinked. The second RT ensures that cDNA recovery is maximal, particularly if RT terminated at a crosslinked site prior to reverse crosslinking. After generating cDNA, the RNA is degraded by addition of RNaseH and RNase cocktail, and the 3'end of the resulting cDNA is ligated to attach an dsDNA oligo containing library amplification sequences for subsequent amplification.

Previously, we performed cDNA (ssDNA) to ssDNA primer ligation which relies on the two single stranded sequences coming together for conversion to a product that can then be amplified for library preparation. To improve the efficiency of cDNA molecules ligated with the Read1 Illumina priming sequence, we perform a "splint" ligation, which involves a chimeric ssDNA-dsDNA adaptor that 
952 contains a random 6mer that anneals to the 3' end of the cDNA and brings the 5' phosphorylated end of 953 the cDNA adapter directly together with the cDNA via annealing. This ligation is performed with $1 \mathrm{x}$ 954 NEB Instant Sticky End Master Mix at $20^{\circ} \mathrm{C}$ for 1 hour. This greatly improves the cDNA tagging and 955 overall RNA yield.

956 Libraries were amplified using Q5 Hot-Start Mastermix (NEB) with primers that add the full Illumina 957 adaptor sequences. After amplification, the libraries are cleaned up using 0.8X SPRI (AMPure XP) and 958 then gel cut using the Zymo Gel Extraction Kit selecting for sizes between $280 \mathrm{bp}-1.3 \mathrm{~kb}$.

959 Sequencing. Sequencing was performed on an Illumina NovaSeq S4 paired-end 150x150 cycle run. For 960 the mES RNA-DNA RD-SPRITE data in this experiment, 144 different SPRITE libraries were 961 generated from two technical replicate SPRITE experiments and were sequenced. Each SPRITE library 962 corresponds to a distinct aliquot during the Proteinase K reverse crosslinking step which is separately 963 amplified with a different barcoded primer, providing an additional round of SPRITE barcoding.

Primers Used for RPM, DPM, and Splint Ligation (IDT):

965

966

967

968

969

970

971

972

973

974

975

976

977

978

979

1. RPM top: /5Phos/rArUrCrArGrCrACTTAGCG TCAG/3SpC3/

2. RPM bottom (internal biotin): /5Phos/TGACTTGC/iBiodT/GACGCTAAGTGCTGAT

3. DPM Phosphorothioate top: /5Phos/AAGACCACCAGATCGGAAGAGCGTCGTG*T* $\mathrm{A} * \mathrm{G} * \mathrm{G} * / 32 \mathrm{MOErG} / *$ Denotes Phosphorothioate bonds

4. DPM bottom (internal biotin): /5Phos/TGACTTGTCATGTCT/iBioT/CCGATCTGGTGGTCTT 5. 2Puni splint top: TACACGACGCTCTTCCGATCT NNNNNN/3SpC3/

6. 2Puni splint bottom: /5Phos/AGA TCG GAA GAG CGT CGT GTA/3SpC3/

Annealing of adaptors. A double-stranded DPM oligo and 2P universal "splint" oligo were generated by annealing the complementary top and bottom strands at equimolar concentrations. Specifically, the oligos were annealed in $1 \mathrm{x}$ Annealing Buffer $\left(0.2 \mathrm{M} \mathrm{LiCl}_{2}, 10 \mathrm{mM}\right.$ Tris- $\mathrm{HCl} \mathrm{pH} \mathrm{7.5)}$ by heating to $95^{\circ} \mathrm{C}$ and then slowly cooling to room temperature $\left(-1^{\circ} \mathrm{C}\right.$ every $\left.10 \mathrm{sec}\right)$ using a thermocycler.

Assessing molecule to bead ratio. We ensured that SPRITE clusters represent bona fide interactions that occur within a cell by mixing human and mouse cells and ensuring that virtually all SPRITE clusters ( $\sim 99 \%)$ represent molecules exclusively from a single species. Specifically, we separately crosslinked HEK293T cells performed a human-mouse mixing RD-SPRITE experiment and identified conditions with 
980 low interspecies mixing (molecules $=$ RNA+DNA instead of DNA). Specifically, for SPRITE clusters 981 containing 2-1000 reads, the percent of interspecies contacts is: 2 beads:molecule $=0.9 \%$ interspecies 982 contacts, 4 beads:molecule $=1.1 \%$ interspecies contacts, 8 beads:molecule $=1.1 \%$ interspecies contacts. 983 We used the 2 beads:molecule ratio for the RD-SPRITE data set generated in this paper.

\section{RD-SPRITE processing pipeline}

986

987

988

989

990

991

992

993

994

995

996

997

998

999

1000

1001

1002

1003

1004

1005

1006

1007

Adapter trimming. Adapters were trimmed from raw paired-end fastq files using Trim Galore! v0.6.2 (https://www.bioinformatics.babraham.ac.uk/projects/trim_galore/) and assessed with Fastqc v0.11.9. Subsequently, the DPM (GATCGGAAGAG) and RPM (ATCAGCACTTA) sequences are trimmed using Cutadapt v2.5 $5^{126}$ from the 5' end of Read 1 along with the 3' end DPM sequences that result from short reads being read through into the barcode (GGTGGTCTTT, GCCTCTTGTT, CCAGGTATTT, TAAGAGAGTT, TTCTCCTCTT, ACCCTCGATT). The additional trimming helps improve read mapping in the end-to-end alignment mode. The SPRITE barcodes of trimmed reads are identified with Barcode ID v1.2.0 (https:/github.com/GuttmanLab/sprite2.0-pipeline) and the ligation efficiency is assessed. Reads with an RPM or a DPM barcode are split into two separate files, to process RNA and DNA reads individually downstream, respectively.

Processing $\boldsymbol{R} \boldsymbol{N} \boldsymbol{A}$ reads. RNA reads were aligned to GRCm38.p6 with the Ensembl GRCm38 v95 gene model annotation using Hisat2 v2.1.0 ${ }^{127}$ with a high penalty for soft-clipping --sp 1000,1000. Unmapped and reads with a low MapQ score (samtools view -bq 20) were filtered out for downstream realignment. Mapped reads were annotated for gene exons and introns with the featureCounts tool from the subread package v1.6.4 using Ensembl GRCm38 v95 gene model annotation and the Repeat and Transposable element annotation from the Hammel lab ${ }^{128}$. Filtered reads were subsequently realigned to our custom collection of repeat sequences using Bowtie v2.3.5 $5^{129}$, only keeping mapped and primary alignment reads.

Processing DNA reads. DNA reads were aligned to GRCm38.p6 using Bowtie2 v2.3.5, filtering out unmapped and reads with a low MapQ score (samtools view -bq 20). Data generated in F1 hybrid cells (pSM33: C57BL/6 × 129SV-Jae or pSM44: $129 \times$ castaneous) were assigned the allele of origin using SNPsplit v0.3.4 $4^{130}$. RepeatMasker ${ }^{131}$ defined regions with milliDev $<=140$ along with blacklisted v2 regions were filtered out using Bedtools v2.29.0 $0^{132}$. 
1008 SPRITE cluster file generation. RNA and DNA reads were merged, and a cluster file was generated for 1009 all downstream analysis. MultiQC v1.6 $6^{133}$ was used to aggregate all reports.

1010 Masked bins. In addition to known repeat containing bins, we manually masked the following bins (mm10 1011 genomic regions: chr2:79490000-79500000, chr11:3119270-3192250, chr15:99734977-99736026, 1012 chr3:5173978-5175025, chr13:58176952-58178051) because we observed a major overrepresentation of 1013 reads in the input samples.

1015 Microscopy imaging

1016 3D-Structured Illumination Microscopy (3D-SIM): 3D-SIM super-resolution imaging was performed 1017 on a DeltaVision OMX-SR system (Cytiva, Marlborough, MA, USA) equipped with a 60x/1.42 NA 1018 Plan Apo oil immersion objective (Olympus, Tokyo, Japan), sCMOS cameras (PCO, Kelheim, 1019 Germany) and $642 \mathrm{~nm}$ diode laser. Image stacks were acquired with z-steps of $125 \mathrm{~nm}$ and with 15 raw 1020 images per plane. The raw data were computationally reconstructed with the soft-WoRx 7.0.0 software 1021 package (Cytiva, Marlborough, MA, USA) using a wiener filter set to 0.002 and channel-specifically 1022 measured optical transfer functions (OTFs) using an immersion oil with a 1.518 refractive index (RI). 1023 32-bit raw datasets were imported to ImageJ and converted to 16-bit stacks.

1024 Immunofluorescence (IF). Cells were grown on coverslips and rinsed with 1xPBS, fixed in 4\% 1025 paraformaldehyde in PBS for 15 minutes at room temperature, rinsed in 1xPBS, and permeabilized with $10260.5 \%$ Triton X-100 in PBS for 10 minutes at room temperature. Cells were either stored at $-20^{\circ} \mathrm{C}$ in $70 \%$ 1027 ethanol or used directly for immunostaining and incubated in blocking solution (0.2\% BSA in PBS) for at 1028 least 1 hour. If stored in 70\% ethanol, cells were re-hydrated prior to staining by washing 3 times in $1 \times$ PBS 1029 and incubated in blocking solution (0.2\% BSA in PBS) for at least 1 hour. Primary antibodies were diluted 1030 in blocking solution (see below) and added to coverslips for 3-5 hours at room temperature incubation. 1031 Cells were washed three times with 0.01\% Triton X-100 in PBS for 5 minutes each and then incubated in 1032 blocking solution containing corresponding secondary antibodies labeled with Alexa fluorophores 1033 (Invitrogen) for 1 hour at room temperature. Next, cells were washed 3 times in 1xPBS for 5 minutes at 1034 room temperature and mounting was done in ProLong Gold with DAPI (Invitrogen, P36935). Images 
1035 were collected on a LSM800 confocal microscope (Zeiss) with a $63 \times$ oil objective. $Z$ sections were taken 1036 every $0.3 \mu \mathrm{m}$. Image visualization and analysis was performed with Icy software and ImageJ software.

1037 Antibodies. Primary antibodies used in the study: anti-Nucleolin (Abcam ab22758 1:500); anti-NPAT 1038 (Abcam ab70595, 1:100); anti-SMN (BD 610646, 1:100); anti-CENP-A (Cell Signaling C51A7, 1:500); 1039 anti-HP1beta (Active Motif 39979, 1:200); anti-Coilin (Abcam Ab-210785, Santa Cruz sc-55594, Santa 1040 Cruz sc-56298, 1:100), all diluted in blocking solution.

1041 RNA Fluorescence in situ Hybridization (RNA-FISH). RNA-FISH performed in this study was based 1042 on the ViewRNA ISH (Thermo Fisher Scientific, QVC0001) protocol with minor modifications. Cells 1043 grown on coverslips were rinsed in 1xPBS, fixed in 4\% paraformaldehyde in 1xPBS for 15 minutes at 1044 room temperature, permeabilized in 0.5\% Triton-100 in the fixative for 10 minutes at room temperature, 1045 rinsed 3 times with $1 \times$ PBS and stored at $-20^{\circ} \mathrm{C}$ in $70 \%$ ethanol until hybridization steps. All the following 1046 steps were performed according to manufacturer's recommendations. Coverslips were mounted with 1047 ProLong Gold with DAPI (Invitrogen, P36935) and stored at $4^{\circ} \mathrm{C}$ until acquisition. For nuclear and 1048 nucleolar RNAs, cells were pre-extracted with $0.5 \%$ ice cold Triton-100 for 3 minutes to remove 1049 cytoplasmic background and fixed as described. All probes used in the study were custom made by 1050 Thermofisher. To test their specificity, we either utilized RNAse treatment prior to RNA-FISH or two 1051 different probes targeting the same RNA. Images were acquired on Zeiss LSM800 confocal microscope 1052 with a 100x glycerol immersion objective lens and Z-sections were taken every $0.3 \mu \mathrm{m}$. Image 1053 visualization and analysis was performed with Icy software and ImageJ software.

1054 RNA FISH for scaRNA and tRNAs were performed with a combined set of probes to increase the signal 1055 of lower abundance RNAs. Specifically, scaRNAs were visualized with two combined probes of scaRNA2 1056 and scaRNA17. tRNAs were visualized using probes targeting tRNA-Arg-TCG-4-1, tRNA-Leu-AAG-31057 1, tRNA-Ile-AAT-1-8, tRNA-Arg-TCT-5-1, tRNA-Leu-CAA-2-1, tRNA-Ile-TAT-2-1, tRNA-Tyr-GTA1058 1-1. tRNA sequences were obtained using the GtRNAdb GRCm38/mm10 predictions (Lowe Lab, $1059 \mathrm{UCSC})^{134,135}$.

$1060 \boldsymbol{R N A - F I S H}$ and $\boldsymbol{I F}$. For immunostaining combined with in situ RNA visualization, we used the 1061 ViewRNA Cell Plus (Thermo Fisher Scientific, 88-19000-99) kit per the manufacturer's protocol with 1062 minor modifications. First immunostaining was performed as described above but all the incubations were 1063 performed in blocking buffer with addition of RNAse inhibitor and all the wash steps were done in RNAse 
1064 free 1xPBS with RNAse inhibitor. Blocking buffer, PBS, RNAse inhibitors are provided in a kit. After 1065 the last wash in 1xPBS, cells underwent post-fixation in $2 \%$ paraformaldehyde on $1 \times$ PBS for $10 \mathrm{~min}$ at 1066 room temperature, were washed 3 times in 1XPBS, and then RNA-FISH protocol was followed as 1067 described above. Images were acquired on the Zeiss LSM800 confocal microscope with a 100x glycerol 1068 immersion objective lens and Z-sections were taken every $0.3 \mu \mathrm{m}$. Image visualization and analysis was 1069 performed with Icy software (http://icy.bioimageanalysis.org/) and ImageJ software 1070 (https://imagej.nih.gov/).

$1071 \boldsymbol{R N A - F I S H}$ for FVP experiments. To compare the relative stability of lncRNAs and pre-mRNAs, we 1072 obtained intron FISH probes for targets of comparable gene length to lncRNAs. This was done to ensure 1073 that any differences in RNA stability upon FVP treatment are not due to differences in the time it takes to 1074 transcribe each RNA. Specifically, we obtained probes for pre-mRNAs that are 57.87kb (Nup188), 73.7kb 1075 (Mbd5), 99.8kb (Abi1), 129.7kb (Ehmt1),131.8kb (Atrx), and 297.2kb (Gtdc1) in length. For lncRNAs, 1076 we obtained probes for RNAs of lengths $53.4 \mathrm{~kb}$ (Tsix), 79.5kb (Dleu2), 93.1kb (Kcnq1 ot1), and 340kb 1077 (Pvt1).

1078 DNA-FISH. DNA-FISH was performed as previously described ${ }^{136}$ with modifications. Cells grown on 1079 coverslips were rinsed with 1xPBS, fixed in 4\% paraformaldehyde in 1xPBS for 15 minutes at room 1080 temperature, permeabilized in $0.5 \%$ Triton-100 in the fixative for 10 minutes at room temperature, rinsed 10813 times with $1 \times$ PBS and stored at $-20^{\circ} \mathrm{C}$ in $70 \%$ ethanol until hybridization steps. Pre-hybridization cells 1082 were dehydrated in $100 \%$ ethanol and dried for 5 minutes at room temperature. 4ul drop of hybridization 1083 mix with probes was spotted on a glass slide and dried coverslips were placed on the drop. Coverslips 1084 were sealed with rubber cement, slides were incubated for 5 minutes at $85^{\circ} \mathrm{C}$, and then incubated overnight 1085 at $37^{\circ} \mathrm{C}$ in humid atmosphere. After hybridization and three washes with $2 x \mathrm{SSC}, 0.05 \%$ Triton-100 and $10861 \mathrm{mg} / \mathrm{ml} \mathrm{PVP}$ in PBS at $50^{\circ} \mathrm{C}$ for 10 minutes, cells were rinsed in 1xPBS and mounted with ProLong Gold 1087 with DAPI (Invitrogen, P36935).

1088 Hybridization buffer consisted of 50\% formamide, 10\% dextran sulphate, 2xSSC, $1 \mathrm{mg} / \mathrm{ml}$ polyvinyl 1089 pyrrolidone (PVP), 0.05\% Triton X-100, $0.5 \mathrm{mg} / \mathrm{ml}$ BSA. $1 \mathrm{mM}$ short oligonucleotides labeled with Cy5 1090 ([CY5]ttttctcgccatattccaggtc) were used as probes against Major Satellites and full-length minor satellite 1091 repeat sequence was used as probes against Minor Satellites. Minor satellite sequence was firstly cloned 1092 to pGEM plasmid and then labeled by PCR reaction with self-made TAMRA dATPs for minor satellites. 
1093 Labeled PCR product was purified with a QIAquick PCR Purification Kit (QIAGEN) and 50ng was mixed 1094 with hybridization buffer. Images were acquired on Zeiss LSM800 confocal microscope with a 63x 1095 glycerol immersion objective lens and Z-sections were taken every $0.3 \mu \mathrm{m}$. Image visualization and 1096 analysis was performed with Icy software and ImageJ software.

1098 Analysis of RNA-DNA contacts

1099 Generating contact profiles. To map the genome-wide localization profile of a specific RNA, we 1100 calculated the contact frequency between the RNA transcript and each region of the genome binned at 1101 various resolutions $(1 \mathrm{Mb}, 100 \mathrm{~kb}$ and $10 \mathrm{~kb})$. Raw contact frequencies were computed by counting the 1102 number of SPRITE clusters in which an RNA transcript and a genomic bin co-occur. We normalized these 1103 raw contacts by weighting each contact by a scaling factor based on the size of its corresponding SPRITE 1104 cluster. Specifically, we enumerate all pairwise contacts within a SPRITE cluster and weight each contact 1105 by $2 / \mathrm{n}$, where $\mathrm{n}$ is the total number of reads within a cluster.

$1106 \boldsymbol{R N A}$ and cluster sizes. RNA-DNA contacts were computed for a range of SPRITE cluster sizes, such as 1107 2-10, 11-100, and 101-1000 reads. We found that different RNAs tend to be most represented in different 1108 clusters sizes - likely reflecting the size of the nuclear compartment that they occupy. For example, 45S 1109 and snoRNAs are most represented in large clusters, while Malat1, snRNAs, and other ncRNAs tend to 1110 be represented in smaller SPRITE clusters. For analyses in this paper we utilized clusters containing 211111000 reads.

1112 Visualizing contact profiles. These methods produce a one-dimensional vector of DNA contact 1113 frequencies for each RNA transcript that we output in bedgraph format and visualize with IGV ${ }^{137}$. To 1114 compare DNA contact profiles between RNA transcripts, we calculated a Pearson correlation coefficient 1115 between the one-dimensional DNA contact vectors for all pairs of RNA transcripts.

1116 Aggregate analysis. To map RNA localization across chromosomes with respect to centromeres and 1117 telomeres (e.g. Terc and satellite ncRNAs), we computed an average localization profile as a function of 1118 distance from the centromere of each chromosomes. To do this, we converted each $1 \mathrm{Mb}$ genomic bin into 1119 a percentile bin from 0 to 100 based on its relative position on its chromosome (from 5' to 3' ends). We 
1120 then calculated the average contact frequency between a given RNA and each percentile bin across all 1121 chromosomes.

1122 Allele specific analysis. To map localization to different alleles, we identified all clusters containing a 1123 given RNA (as above) and quantified the number of DNA reads uniquely mapping to each allele using 1124 allele specific alignments. Allele specific RNA-DNA contact frequencies were normalized by overall 1125 genomic read coverage for each allele to account for differences in coverage for each allele.

1126 Nucleolar hub RNA-DNA contacts. We observe enrichment of pre-rRNAs and other nucleolar hub RNAs 1127 on chromosomes containing 45S ribosomal DNA (rDNA). Specifically, rDNA genes are contained on the 1128 centromere-proximal regions of chromosomes $12,15,16,18$, and 19 in mouse ES cells. We previously 1129 showed that regions on these chromosomes organize around nucleoli in the majority of cells imaged with 1130 DNA FISH combined with immunofluorescence for Nucleolin ${ }^{54}$. We also observed nucleolar hub RNAs 1131 enriched on other genomic regions corresponding to centromere-proximal DNA and transcriptionally 1132 inactive, gene poor regions. We previously showed that these genomic regions are organized proximal to 1133 the nucleolus using SPRITE and microscopy ${ }^{54}$.

\section{Analysis of RNA-RNA contacts}

$1136 \boldsymbol{R N A}-\boldsymbol{R} \boldsymbol{N} \boldsymbol{A}$ contact matrices. We computed contact frequency between each RNA-RNA pair by counting 1137 the number of SPRITE clusters containing two different RNAs. To account for coverage differences in 1138 individual RNAs, we normalized this matrix using a matrix balancing normalization approach as 1139 previously described ${ }^{138}$. Briefly, this approach works by ensuring the rows and columns of a symmetric 1140 matrix add up to 1 . In this way, RNA abundance does not dominate the overall strength of the contact 1141 matrix. For multi-copy RNAs (e.g. repeat-encoded RNAs, ribosomal RNA, tRNAs), all reads mapping to 1142 a given RNA were collapsed. Specifically, multi-copy RNA reads mapping to either the mm10 genome 1143 annotated using repeat masker or a custom repeat genome consensus were collapsed.

$1144 \boldsymbol{R N A}$ Hubs. RNAs in each hub were identified using hierarchical clustering of the RNA-RNA contact 1145 matrix. Specifically, each hub corresponds to sets of RNAs with high contacts with other RNAs within 1146 the same hub, but low contacts with other RNAs in other hubs. 
Mapping intron versus exon $R \boldsymbol{N} \boldsymbol{A}-\boldsymbol{R} \boldsymbol{N} \boldsymbol{A}$ contacts. To explore the differential RNA contacts that occur within nascent pre-mRNA and mature mRNAs, we focused on the intronic regions and exonic regions of mRNAs respectively. We retained all intronic or exonic regions that were contained in at least 100

1150 independent SPRITE clusters. We then generate contact matrices between splicing non-coding RNAs (U1, $1151 \mathrm{U} 2, \mathrm{U} 4, \mathrm{U} 5, \mathrm{U} 6)$ and translation non-coding RNAs (18S, 28S, 5S, 5.8S) and these mRNA exons, and 1152 introns. We performed a matrix balancing normalization (ICE normalization ${ }^{138}$ ) on this symmetric contact 1153 matrix and plotted splicing RNAs and translation RNAs (columns) versus mRNA exons and introns 1154 (rows).

1155 Identifying unannotated scaRNAs. We calculated the weighted contact frequency of how often a given 1156 RNA contacts scaRNA2. Many of the top hits correspond to Mus musculus (mm10) annotated scaRNAs 1157 (e.g. scaRNA9, scaRNA10, scaRNA6, scaRNA7, scaRNA1, scaRNA17, and scaRNA13). Other hits 1158 include regions within mRNA introns. We performed BLAST-like Alignment Tool (BLAT, 1159 https://genome.ucsc.edu/cgi-bin/hgBlat) on other top hits contacting scaRNA2, including the Trrap intron 1160 region and Gon411 intron region and found they are homologous to human scaRNA28 and scaRNA26A, 1161 respectively. Specifically, the Trrap region in mm10 homologous to scaRNA28 is chr5:1447713391162144771531 and the Gon4l region in mm10 homologous to scaRNA26A is chr3:88880319-88880467.

\section{Analysis of multiway RNA and DNA SPRITE contacts}

1164 Generating RNA-DNA-DNA Contact Matrices for SPRITE clusters containing an individual or 1165 multiple RNAs. To analyze higher-order RNA and DNA contacts in the SPRITE clusters, we generated 1166 DNA-DNA contact frequency maps in the presence of specific sets of RNA transcripts. To generate these 1167 DNA-DNA contact maps, we first obtained the subset of SPRITE clusters that contained an RNA 1168 transcript or multiple transcripts of interest (e.g., nucleolar RNAs, spliceosomal RNAs, scaRNAs satellite 1169 RNAs, IncRNA). We then calculated DNA-DNA contact maps for each subset of SPRITE clusters at $1170100 \mathrm{~kb}$ and $1 \mathrm{Mb}$ resolution by determining the number of clusters in which each pair of genomic bins co1171 occur. Raw contacts were normalized by SPRITE cluster size by dividing each contact by the total number 1172 of reads in the corresponding SPRITE cluster as described above. This resulted in genome-wide DNA1173 DNA contact frequency maps for each set of RNA transcripts of interest.

1174 Aggregate inter-chromosomal maps. For satellite-derived ncRNAs, we also calculated a mean inter1175 chromosomal contact frequency map. To do this, we converted each $1 \mathrm{Mb}$ genomic bin into a percentile 
1176 bin from 0 to 100 based on its chromosomal position, where the 5' end is 0 and the 3 ' end is 100 . We then 1177 calculated the contact frequency between all pairs of percentile bins for all pairs of chromosomes. We 1178 used these values to calculate a mean inter-chromosomal contact frequency map, which reflects the 1179 average contact frequency between each pair of percentile bins between all pairs of chromosomes.

1181 Satellite-derived ncRNA knockdowns and HP1 measurements

1182 LNA transfections. LNA antisense oligonucleotides designed against Major Satellite and Minor Satellite 1183 were transfected using Lipofectamine ${ }^{\mathrm{TM}}$ RNAiMAX Transfection Reagent according to manufacturer 1184 protocol (Thermo Fisher Scientific \#13778030). We designed LNAs targeting the forward and reverse 1185 strand of the satellite-derived RNAs. These probes, targeting distinct regions of the transcript, were mixed 1186 together to a final concentration of $10 \mathrm{uM}$ each and $5 \mathrm{ul}$ of the mix was transfected to each well of a $24-$ 1187 well plate containing cells. As a control, non-targeting LNA were transfected at the same concentrations. 1188 After $48 \mathrm{~h}$ or $72 \mathrm{~h}$ in culture, cells were used for further procedures. KD for both LNA were confirmed by 1189 RT-qPCRs (Supplemental Figure 5C-D).

1190 LNA sequences. LNAs were designed by Qiagen. The following sequences were used. Minor Satellite 1191 (forward): ACTCACTCATCTAATA, Minor Satellite (reverse): TGGCAAGACAACTGAA, Major 1192 Satellite (forward): AGGTCCTTCAGTGTGC, Major Satellite (reverse): ACATTCGTTGGAAACG

1193 Reverse transcription and quantitative PCR (RT-qPCR). Total RNA was extracted from ES cells with 1194 Silane beads (Sigma) according to manufacturer conditions and treated with Turbo DNase (Life 1195 Technologies) for $15 \mathrm{~min}$ at $37 \mathrm{C}$ to remove genomic DNA. RT reactions were performed according to 1196 Superscript II protocol (Thermo Fisher Scientific \#18064022) with random 9mer. qPCRs were performed 1197 in technical replicates using a Roche Lightcycler and a representative of three biological replicates is 1198 shown. Plots were generated using GraphPad software. ddCt values were calculated by normalizing $\mathrm{Ct}$ 1199 values to GAPDH and to samples transfected with control LNA to compare gene expression differences 1200 between samples.

1201 qPCR primers used for analysis.

1202

GAPDH:CATGGCCTTCCGTGTTCCTA

GCCTGCTTCACCACCTTCTT

1203

MinS_1: GAACATATTAGATGAGTGAGTTAC GTTCTACAAATCCCGTTTCCAAC 
MinS 2: GATGGAAAATGATAAAAACC CATCTAATATGTTCTACAGTGTGG MajS_1: GACGACTTGAAAAATGACGAAATC CATATTCCAGGTCCTTCAGTGTGC MajS_2: GCACACTGAAGGACCTGGAATATG GATTTCGTCATTTTTCAAGTCGTC

Image analysis of HP1 foci. Image visualization and analysis was performed with Icy software and ImageJ software with a minimum of 10 cells observed per condition. For HP1 foci quantification, we computed a binary mask based on relative intensity threshold ( $>100$ for HP1ß staining replicate $1,>120$ for HP1ß replicate 2) in which the relative signal intensity was set from 10 to 200 .

\section{Mapping IncRNA localization}

Defining IncRNAs. We used Gencode release 95 (GRCm38.p6, https://ftp.ensembl.org/pub/release95/gtf/mus_musculus/Mus_musculus.GRCm38.95.gtf.gz) to define all lncRNAs in this study. Specifically, we included all annotations with the "lincRNA" or "antisense" biotypes to define all lncRNAs. For example, lncRNAs such as Tsix, Airn, and Kcnq1ot1 are annotated as "antisense" rather than "lincRNA". We included all lncRNAs that contained coverage in our mouse ES data by filtering the list to those that were contained in at least 10 SPRITE clusters. This yielded a list of 642 lncRNAs.

Calculation of chromatin enrichment scores. To determine the extent to which RNA transcripts are in contact with chromatin, we calculated a chromatin enrichment score for each RNA transcript. The chromatin enrichment score is computed as the ratio of the number of SPRITE clusters containing a given RNA that also contains DNA ("chromatin bound") relative to all SPRITE clusters containing the RNA transcript. We normalize these counts by the SPRITE cluster size in which it was observed. We determined an "expected" DNA to RNA contact ratio by calculating mean DNA to RNA contact ratio across all RNA transcripts. Chromatin enrichment scores were calculated as the natural log of the observed DNA to RNA contact ratio divided by the expected ratio. Positive chromatin enrichment scores indicate RNA transcripts with higher ratios of DNA to RNA contacts than the mean. We performed a similar analysis to calculate enrichment scores for different sets of RNA transcripts. For example, we compute a ribosomal RNA enrichment score based on the ratio of ribosomal RNA contacts to all RNA contacts for a given RNA transcript.

IncRNA RNA-DNA genome wide heatmap. We plotted these 642 lncRNAs across the genome at $10 \mathrm{Mb}$ resolution. For each lncRNA, we computed the number of SPRITE clusters that co-occur within each 
$123310 \mathrm{Mb}$ bin. We then normalized this count by the average contacts across all genomic bins. We refer to 1234 this ratio as an enrichment score. This enrichment score is intrinsically normalized for the different 1235 expression levels of different lncRNAs. We plotted all bins that have an enrichment value greater than 51236 fold. We zoomed in on selected examples and plotted them across the entire genome at $1 \mathrm{Mb}$ resolution. 1237 In these examples, we plotted the enrichment scores across all values as a continuous bedgraph in IGV.

1238 Calculation of IncRNAs enriched around their transcriptional loci. Using these values, we defined a 1239 lncRNA as enriched in proximity to its transcriptional locus if it was $>20$-fold enriched within the $10 \mathrm{Mb}$ 1240 bin containing its transcriptional loci. At this cutoff, lncRNAs that have very broad distribution patterns 1241 across the genome such as Malat1 are excluded, while the vast majority of lncRNAs (596 lncRNAs, $124292.8 \%$ ) are highly enriched around their transcriptional loci.

1243 Visualizing proportion of IncRNAs or $\boldsymbol{m} \boldsymbol{R} \mathbf{N} A s$ on chromatin. To visually compare the fraction of 1244 different RNAs that are retained on chromatin across the genome, we computed a weighted score 1245 accounting for the counts within a given genomic bin relative to the total fraction of SPRITE clusters 1246 contained off chromatin. Specifically, we identified all SPRITE clusters containing a given RNA and 1247 computed the number that also contained a DNA read (on chromatin count) and the number that do not 1248 contain DNA (off chromatin count). We computed a score for each genomic bin defined as the number of 1249 SPRITE clusters containing an RNA and genomic bin by dividing this count by the total number of 1250 SPRITE clusters containing the same RNA that did not have a paired DNA read (off-DNA count). We 1251 multiplied this number by 100 to linearly scale values. This score accounts for different abundance levels 1252 of different RNAs allowing us to compare them directly to each other and accounts for the proportion of 1253 the RNA that is present on chromatin versus off-chromatin.

1254 Generating nuclear structure models of IncRNA localization. To visualize the localization of lncRNAs 1255 in 3D, we generated 3D models of the genome based on SPRITE DNA-DNA contacts. We modeled each 1256 chromosome as a linear polymer composed of $\mathrm{N}$ monomers, where $\mathrm{N}$ is the number of $1 \mathrm{Mb}$ bins on the 1257 chromosome. Each chromosome polymer is initialized as a random walk, and then a Brownian dynamics 1258 simulation is performed on all chromosomes using an energy function composed of the following forces: 1259 1) a harmonic bond force between adjacent monomers, 2) a spherical confinement force, 3) a repulsive 1260 force to prevent monomers from overlapping, 4) an attractive force based on SPRITE contact frequencies 1261 to ensure that preferential contacts determined by SPRITE are accurately reflected by the models. 
1262 Simulations were performed using the open-source molecular simulation software OpenMM. The outputs 1263 of simulations were visualized using Pymol 2 (pymol.org/2). Chromosomes were visualized as cartoon 1264 tubes and lncRNAs were visualized by drawing a surface over the genomic regions where lncRNA 1265 enrichment was greater than 50-fold over background.

1266 FVP treatment and analysis. GRO-seq data from Jonkers et al. ${ }^{139}$ were obtained from NCBI GEO 1267 (accession GSE48895) and aligned to mm10 using HISAT2. Raw read counts were determined for each 1268 gene using deepTools module multiBamSummary for untreated and 50 min FVP conditions. Raw read 1269 counts were converted to transcripts per million (TPM) values using a custom Python script, and fold 1270 change in TPM was calculated for each gene by dividing 50 min FVP TPM values by untreated TPM 1271 values. Cumulative distribution plots were generated using R and box-and-whisker plots were generated 1272 using prism.

\section{Kcnq10t1 protein binding, perturbations, and gene expression measurements}

1275

1276

1278

Kcnq1ot1 CRISPR interference. dCas9-4XSID cells were transfected using multiplexed gRNA vector constructs, containing an episomal polyoma origin of replication, puromycin resistance driven by a PGK promoter, and four tandem U6-gRNA cassettes, allowing for simultaneous expression of four sgRNAs. Negative control gRNA sequences recognizing the Saccharomyces cerevisiae Upstream Activation Sequence (UAS) and the Tetracycline Response Element (TRE) were multiplexed together (referred to as sgTUUT; gRNAs are as follows: TCTCTATCACTGATAGGGAG, GAGGACAGTACTCCGCTCGG, GCGGAGTACTGTCCTCCGAG, and TCTCTATCACTGATAGGGAG). Four gRNA sequences targeting the Kcnq1ot1 promoter were multiplexed together (referred to as sgKcnq1ot1; gRNAs are as follows: $\quad$ GCCTAGCCGTTGTCGCTAGG, GCCCTGTACTGCATTGAGGT, GCCTGCACAGTAGGATTCCA, and GGAGGATGGGTCGAGTGGCT).

dCas9-4XSID cells were transfected with either sgTUUT or sgKcnq1ot1 and selected for three days with $1 \mu \mathrm{g} / \mathrm{ml}$ of puromycin in standard $2 \mathrm{i}$ culture conditions. Cells were subsequently passaged and maintained in $0.5 \mu \mathrm{g} / \mathrm{ml}$ puromycin for an additional 7 days prior to RNA harvesting. Data presented are from two separate transfections and biological replicates. 
1289 SHARP binding to Kcnq1ot1 RNA. We transfected an expression vector containing full-length SHARP 1290 with an N-terminal Halo-FLAG (HF) fusion protein into mouse ES cells containing a doxycycline 1291 inducible Xist gene. Cells were washed once with PBS and then crosslinked on ice using $0.25 \mathrm{~J} \mathrm{~cm}-2$ 1292 (UV2.5k) of UV at $254 \mathrm{~nm}$ in a Spectrolinker UV Crosslinker. Cells were then scraped from culture 1293 dishes, washed once with PBS, pelleted by centrifugation at 1,500g for $4 \mathrm{~min}$, and flash-frozen in liquid 1294 nitrogen for storage at $-80^{\circ} \mathrm{C}$. We lysed batches of 5 million cells by completely resuspending frozen cell 1295 pellets in $1 \mathrm{~mL}$ of ice cold iCLIP lysis buffer (50 mM Hepes, pH 7.4, $100 \mathrm{mM} \mathrm{NaCl,} \mathrm{1 \%} \mathrm{NP-40,} \mathrm{0.1 \%}$ 1296 SDS, 0.5\% Sodium Deoxycholate) supplemented with 1X Protease Inhibitor Cocktail (Promega), 200 U 1297 of Murine RNase Inhibitor (New England Biolabs), 20 U Turbo DNase (Ambion), and 1X 1298 Manganese/Calcium Mix (0.5mM CaCl2, $2.5 \mathrm{mM} \mathrm{MnC12).} \mathrm{Samples} \mathrm{were} \mathrm{incubated} \mathrm{on} \mathrm{ice} \mathrm{for} 10$ minutes 1299 to allow lysis to proceed. The lysates were then incubated at $37^{\circ} \mathrm{C}$ for 10 minutes at $1150 \mathrm{rpm}$ shaking on 1300 a Thermomixer (Eppendorf). Lysates were cleared by centrifugation at 15,000g for 2 minutes. The 1301 supernatant was collected and kept on ice until bound to the HaloLink Resin.

1302 We used $200 \mu \mathrm{L}$ of $25 \%$ HaloLink Resin $(50 \mu \mathrm{L}$ of HaloLink Resin total) per 5 million cells. Resin was 1303 washed three times with $2 \mathrm{~mL}$ of $1 \mathrm{X}$ TBS $(50 \mathrm{mM}$ Tris $\mathrm{pH} 7.5,150 \mathrm{mM} \mathrm{NaCl})$ and incubated in $1 \mathrm{X}$ 1304 Blocking Buffer (50 mM HEPES, pH 7.5, $10 \mu \mathrm{g} / \mathrm{mL}$ Random 9-mer, $100 \mu \mathrm{g} / \mathrm{mL}$ BSA) for 20 minutes at 1305 room temperature with continuous rotation. After the incubation, resin was washed three times with $1 \mathrm{X}$ 1306 TBS. The cleared lysate was mixed with $50 \mu \mathrm{l}$ of HaloLink Resin and incubated at $4{ }^{\circ} \mathrm{C}$ for $3-16 \mathrm{hrs}$ with 1307 continuous rotation. The captured protein bound to resin was washed three times with iCLIP lysis buffer 1308 at room temperature and then washed three times at $90^{\circ} \mathrm{C}$ for 2 minutes while shaking at $1200 \mathrm{rpm}$ with 1309 each of the following buffers: 1X ProK/NLS buffer (50 mM HEPES, pH 7.5, 2\% NLS, 10 mM EDTA, 1310 0.1\% NP-40, 10 mM DTT), High Salt Buffer (50 mM HEPES, pH 7.5, 10 mM EDTA, 0.1\% NP-40, 1M $1311 \mathrm{NaCl}$ ), 8M Urea Buffer (50 mM HEPES, pH 7.5, 10 mM EDTA, 0.1\% NP-40, 8 M Urea), and Tween 1312 buffer (50 mM HEPES, pH 7.5, 0.1\% Tween 20, $10 \mathrm{mM}$ EDTA). Finally, we adjusted the buffer by 1313 washing with Elution Buffer (50 mM HEPES, pH 7.5, $0.5 \mathrm{mM}$ EDTA, 0.1\% NP-40) three times at $30^{\circ} \mathrm{C}$. 1314 The resin was resuspended in $83 \mu \mathrm{L}$ of Elution Buffer and split into a $75 \mu \mathrm{L}$ (ProK elution) and $8 \mu \mathrm{L}$ (TEV 1315 elution) reaction. $25 \mu \mathrm{L}$ of $4 \mathrm{X}$ ProK/NLS Buffer and $10 \mu \mathrm{L}$ of ProK were added to the ProK elution tube 1316 and the sample was incubated at $50^{\circ} \mathrm{C}$ for 30 minutes while shaking at $1200 \mathrm{rpm} .2 .3 \mu \mathrm{L}$ of ProTEV Plus 1317 Protease (Promega) was added to the TEV Elution and the sample was incubated at $30^{\circ} \mathrm{C}$ for 30 minutes 1318 while shaking at $1200 \mathrm{rpm}$. 
1319 For each experiment, we ensured that we successfully purified the Halo-tagged protein. To do this, the 1320 TEV elution sample was mixed with 1X LDS Sample Buffer (Invitrogen) and 1X Reducing Agent 1321 (Invitrogen) and heated for 6 minutes at $70^{\circ} \mathrm{C}$. The sample was run on a 3-8\% Tris Acetate Gel (Invitrogen) 1322 for 1 hour at $150 \mathrm{~V}$. The gel was transferred to a nitrocellulose membrane using an iBlot Transfer Device 1323 (Invitrogen). The nitrocellulose membrane was blocked with Odyssey Blocking Buffer (LI-COR) for 30 1324 minutes. We incubated the membrane in Anti-FLAG mouse monoclonal Antibody (Sigma, F3166) and 1325 V5 rabbit polyclonal antibody (Santa Cruz, sc-83849-R) at a 1:2500 dilution for 2 hours at room 1326 temperature to detect the protein. We visualized the protein by incubating the membrane in 1:17,500 1327 dilution of both IRDye 800CW Goat anti-Rabbit IgG (LI-COR, 925-32210) and IRDYE 680DR Goat 1328 anti-Mouse IgG (LI-COR, 925-68070) for 1 hour at room temperature followed by imaging on a LICOR 1329 Odyssey.

1330 RNA was purified from the Proteinase K elution sample and an RNA-Seq library was constructed as 1331 previously described. Briefly, after proteinase K elution, the RNA was dephosphorylated (Fast AP) and 1332 cyclic phosphates removed (T4 PNK) and then cleaned up on Silane beads as previously described. The 1333 RNA was then ligated to an RNA adapter containing a RT primer binding site. The ligated RNA was 1334 reverse transcribed (RT) into cDNA, the RNA was degraded using $\mathrm{NaOH}$, and a second adapter was 1335 ligated to the single stranded cDNA. The DNA was amplified and Illumina sequencing adaptors were 1336 added by PCR using primers that are complementary to the 3' and 5' adapters. The molarity of PCR 1337 amplified libraries was measured by Agilent Tapestation High Sensitivity DNA screentapes and all 1338 samples were pooled at equal molarity. The pool was then purified and size selected on a $2 \%$ agarose gel 1339 and cut between 150-700 nts. The final libraries were measured by Agilent Bioanalyzer and Qubit high 1340 sensitivity DNA to determine the loading density of the final pooled sample. Pooled samples were paired1341 end sequenced on an Illumina HiSeq 2500 with read length 35 x 35nts.

1342 Sequencing reads were trimmed to remove adaptor sequences and any bases containing a quality scores $1343<10$ using Trimmomatic ${ }^{140}$. We filtered out all read-pairs where either read was trimmed to $<25$ 1344 nucleotides. We excluded PCR duplicates using the FastUniq tool ${ }^{141}$. The remaining reads were then 1345 aligned to Ribosomal RNAs (rRNAs) using the Tagdust program ${ }^{142}$ with a database of $18 \mathrm{~S}, 28 \mathrm{~S}, 45 \mathrm{~S}, 5 \mathrm{~S}$, 1346 5.8S sequences. TagDust was chosen because it allowed more permissive alignments to rRNA reads that 1347 contained mismatches and indels due to RT errors induced by rRNA post-transcriptional modifications. 
1348 The remaining reads were then aligned to the mouse genome using STAR aligner ${ }^{143}$. Only reads that 1349 mapped uniquely in the genome were kept for further analysis.

1350 Genetic deletion of SHARP Binding Site in Kcnq1ot1. F1 2-1 line were CRISPR-targeted with gRNAs 1351 targeting the SHARP-Binding Site (SBS) (SHARP Binding Site Coordinates: mm10 - chr7:143,295,7891352 143,296,455; gRNA sequences were ATGCACCATCATAGACCACG and 1353 TCATAGCCTCCCCCTCCTCG). Following selection using $1 \mu \mathrm{g} / \mathrm{ml}$ of puromycin in standard $2 \mathrm{i}$ culture 1354 conditions, transfected cells were allowed to recover in standard $2 \mathrm{i}$ media prior to sub-cloning. Clone were 1355 subsequently screened using genomic DNA PCR, using primers flanking the deletion region 1356 (CAGCATCTGTCCAATCAACAG and GCAAAATACGAGAACTGAGCC respectively). In contrast 1357 to the wild type $1048 \mathrm{bp}$ band, successfully targeted alleles would produce 305bp band. Sub-clones 1358 homozygous for the targeted allele were subject to RT-qPCR and GAPDH-normalized gene expression 1359 was further normalized to the F1 parent line).

1360 HDAC inhibitor treatment. The inducible Kcnq1 otl cell line were treated with either DMSO (control) or $13615 \mu \mathrm{M}$ TSA in fresh $2 \mathrm{i}$ media or $2 \mu \mathrm{g} / \mathrm{ml}$ doxycycline in standard $2 \mathrm{i}$. RNA was extracted, reverse transcribed, 1362 and qPCR was performed. CT values were normalized to GAPDH to compare gene expression differences 1363 between induced and non-induced samples within the same pharmacologic condition (i.e. GAPDH1364 normalized "Induced DMSO" to GAPDH-normalized "Non-Induced DMSO Vehicle) to generate fold 1365 gene expression ratios. RT-qPCR data presented is summarized from two separate replicate experiments 


\section{REFERENCES}

1367 1. Strom, A. R. \& Brangwynne, C. P. The liquid nucleome - phase transitions in the nucleus at a 1368 glance. Journal of cell science (2019). doi:10.1242/jcs.235093

1369 2. Pombo, A. \& Dillon, N. Three-dimensional genome architecture: players and mechanisms. Nat. Rev. Mol. Cell Biol. 16, 245-257 (2015).

1371 3. Bonev, B. \& Cavalli, G. Organization and function of the 3D genome. Nat. Rev. Genet. 17, 772$1372772(2016)$.

1373 4. Gibcus, J. H. \& Dekker, J. The Hierarchy of the 3D Genome. Mol. Cell 49, 773-782 (2013).

1374 5. Meshorer, E. \& Misteli, T. Chromatin in pluripotent embryonic stem cells and differentiation. 1375 Nat. Rev. Mol. Cell Biol. 7, 540-546 (2006).

1376 6. Dundr, M. \& Misteli, T. Biogenesis of nuclear bodies. Cold Spring Harbor perspectives in 1377 biology 2, (2010).

1378 7. Dekker, J. et al. The 4D nucleome project. Nature (2017). doi:10.1038/nature23884

1379 8. Phillips-Cremins, J. E. Unraveling architecture of the pluripotent genome. Current Opinion in $1380 \quad$ Cell Biology 28, 96-104 (2014).

1381 9. Pederson, T. The nucleolus. Cold Spring Harb. Perspect. Biol. 3, 1-15 (2011).

1382 10. Spector, D. L. \& Lamond, A. I. Nuclear speckles. Cold Spring Harb. Perspect. Biol. 3, 1-12 1383 (2011).

1384 11. Guo, Y. E. et al. Pol II phosphorylation regulates a switch between transcriptional and splicing condensates. Nature (2019). doi:10.1038/s41586-019-1464-0

12. Cho, W. K. et al. Mediator and RNA polymerase II clusters associate in transcription-dependent condensates. Science (80-. ). (2018). doi:10.1126/science.aar4199

13. Nickerson, J. A., Krochmalnic, G., Wan, K. M. \& Penman, S. Chromatin architecture and nuclear RNA. Proc. Natl. Acad. Sci. U. S. A. (1989). doi:10.1073/pnas.86.1.177 
1390 14. Melé, M. \& Rinn, J. L. 'Cat's Cradling' the 3D Genome by the Act of LncRNA Transcription. Molecular Cell (2016). doi:10.1016/j.molcel.2016.05.011

15. Rinn, J. L. \& Guttman, M. RNA and dynamic nuclear organization. Science (80-. ). 345, 12401241 (2014).

16. Quinodoz, S. \& Guttman, M. Long noncoding RNAs: an emerging link between gene regulation and nuclear organization. Trends in cell biology 24, 651-663 (2014).

17. Hall, L. L. \& Lawrence, J. B. RNA as a fundamental component of interphase chromosomes: Could repeats prove key? Current Opinion in Genetics and Development (2016). doi:10.1016/j.gde.2016.04.005

18. Nozawa, R. S. \& Gilbert, N. RNA: Nuclear Glue for Folding the Genome. Trends in Cell Biology (2019). doi:10.1016/j.tcb.2018.12.003

1401 19. Guttman, M. et al. Chromatin signature reveals over a thousand highly conserved large noncoding RNAs in mammals. Nature (2009). doi:10.1038/nature07672

1403 20. Cabili, M. et al. Integrative annotation of human large intergenic noncoding RNAs reveals global 1404 properties and specific subclasses. Genes Dev. (2011). doi:10.1101/gad.17446611

1405 21. Frankish, A. et al. GENCODE reference annotation for the human and mouse genomes. Nucleic Acids Res. (2019). doi:10.1093/nar/gky955

22. Cech, T. R. \& Steitz, J. A. The noncoding RNA revolution - Trashing old rules to forge new ones. Cell (2014). doi:10.1016/j.cell.2014.03.008

23. Rinn, J. L. \& Chang, H. Y. Genome Regulation by Long Noncoding RNAs. Annu. Rev. Biochem. (2012). doi:10.1146/annurev-biochem-051410-092902

1411 24. Black, D. L. Mechanisms of Alternative Pre-Messenger RNA Splicing. Annu. Rev. Biochem. (2003). doi:10.1146/annurev.biochem.72.121801.161720

1413 25. Nilsen, T. W. \& Graveley, B. R. Expansion of the eukaryotic proteome by alternative splicing. 1414 Nature (2010). doi:10.1038/nature08909 
1415 26. Watkins, N. J. \& Bohnsack, M. T. The box C/D and H/ACA snoRNPs: Key players in the modification, processing and the dynamic folding of ribosomal RNA. Wiley Interdisciplinary

1418 27. Kiss-László, Z., Henry, Y., Bachellerie, J. P., Caizergues-Ferrer, M. \& Kiss, T. Site-specific 1419 ribose methylation of preribosomal RNA: A novel function for small nucleolar RNAs. Cell 1420 (1996). doi:10.1016/S0092-8674(00)81308-2

1421 28. Ni, J., Tien, A. L. \& Fournier, M. J. Small nucleolar RNAs direct site-specific synthesis of 1422

29. Spycher, C. et al. 3' end processing of mouse histone pre-mRNA: Evidence for additional base1424 pairing between U7 snRNA and pre-mRNA. Nucleic Acids Res. (1994). doi:10.1093/nar/22.20.4023

30. Mowry, K. L. \& Steitz, J. A. Identification of the human U7 snRNP as one of several factors involved in the 3' end maturation of histone premessenger RNA's. Science (80-. ). (1987). doi:10.1126/science. 2825355

31. Marzluff, W. F. \& Koreski, K. P. Birth and Death of Histone mRNAs. Trends in Genetics (2017). doi:10.1016/j.tig.2017.07.014

32. Kolev, N. G. \& Steitz, J. A. Symplekin and multiple other polyadenylation factors participate in 3'-end maturation of histone mRNAs. Genes Dev. (2005). doi:10.1101/gad.1371105

33. Pandey, R. R. et al. Kcnq1ot1 Antisense Noncoding RNA Mediates Lineage-Specific

34. Mancini-DiNardo, D., Steele, S. J. S., Levorse, J. M., Ingram, R. S. \& Tilghman, S. M. Elongation of the Kcnq1ot1 transcript is required for genomic imprinting of neighboring genes. Genes Dev. (2006). doi:10.1101/gad.1416906 X Chromosome Inactivation. Annu. Rev. Genet. (2002). 
doi:10.1146/annurev.genet.36.042902.092433

1442 36. Quaresma, A. J. C., Bugai, A. \& Barboric, M. Cracking the control of RNA polymerase II

1444 37. Zhou, Q., Li, T. \& Price, D. H. RNA Polymerase II Elongation Control. Annu. Rev. Biochem. (2012). doi:10.1146/annurev-biochem-052610-095910

1446 38. Egloff, S., Studniarek, C. \& Kiss, T. 7SK small nuclear RNA, a multifunctional transcriptional 1447 regulatory RNA with gene-specific features. Transcription (2018).

1448 doi:10.1080/21541264.2017.1344346

1449 39. Carmo-Fonseca, M. \& Rino, J. RNA seeds nuclear bodies. Nat. Cell Biol. (2011).

1450 doi:10.1038/ncb0211-110

1451 40. Shevtsov, S. P. \& Dundr, M. Nucleation of nuclear bodies by RNA. Nat. Cell Biol. (2011). 1452 doi:10.1038/ncb2157

1453 41. Andersen, J. S. et al. Nucleolar proteome dynamics. Nature (2005). doi:10.1038/nature03207

1454 42. Boisvert, F.-M., van Koningsbruggen, S., Navascués, J. \& Lamond, A. I. The multifunctional 1455 nucleolus. Nat. Rev. Mol. Cell Biol. 8, 574-585 (2007).

1456 43. Kresoja-Rakic, J. \& Santoro, R. Nucleolus and rRNA Gene Chromatin in Early Embryo 1457 Development. Trends in Genetics (2019). doi:10.1016/j.tig.2019.06.005

1458 44. Wutz, A. \& Jaenisch, R. A shift from reversible to irreversible X inactivation is triggered during 1459 ES cell differentiation. Mol. Cell (2000). doi:10.1016/S1097-2765(00)80248-8

1460 45. Chaumeil, J., Le Baccon, P., Wutz, A. \& Heard, E. A novel role for Xist RNA in the formation of 1461 1462 a repressive nuclear compartment into which genes are recruited when silenced. Genes Dev. 20 , 2223-2237 (2006).

1463 46. Engreitz, J. M. et al. The Xist lncRNA Exploits Three-Dimensional Genome Architecture to 1464 Spread Across the X Chromosome. Science (80-. ). 341, 1237973-1237973 (2013). 
1465 47. Tripathi, V. et al. The nuclear-retained noncoding RNA MALAT1 regulates alternative splicing by modulating SR splicing factor phosphorylation. Mol. Cell (2010). doi:10.1016/j.molcel.2010.08.011

1468

48. Melé, M. \& Rinn, J. L. 'Cat's Cradling' the 3D Genome by the Act of LncRNA Transcription. Molecular Cell 62, 657-664 (2016).

49. Bell, J. C. et al. Chromatin-associated RNA sequencing (ChAR-seq) maps genome-wide RNA-toDNA contacts. Elife (2018). doi:10.7554/eLife.27024

50. Li, X. et al. GRID-seq reveals the global RNA-chromatin interactome. Nat. Biotechnol. (2017). doi: $10.1038 /$ nbt.3968

51. Yan, Z. et al. Genome-wide colocalization of RNA-DNA interactions and fusion RNA pairs. Proc. Natl. Acad. Sci. U. S. A. (2019). doi:10.1073/pnas.1819788116

52. Sridhar, B. et al. Systematic Mapping of RNA-Chromatin Interactions In Vivo. Curr. Biol. (2017). doi:10.1016/j.cub.2017.01.011

53. Bonetti, A. et al. RADICL-seq identifies general and cell type-specific principles of genome-wide RNA-chromatin interactions. bioRxiv (2019). doi:10.1101/681924

54. Quinodoz, S. A. et al. Higher-Order Inter-chromosomal Hubs Shape 3D Genome Organization in the Nucleus. Cell 174, 744-757.e24 (2018).

55. Quinodoz, S. A. et al. Higher-Order Inter-chromosomal Hubs Shape 3D Genome Organization in the Nucleus. Cell (2018). doi:10.1016/j.cell.2018.05.024

56. Simon, M. D. et al. High-resolution Xist binding maps reveal two-step spreading during Xchromosome inactivation. Nature (2013). doi:10.1038/nature12719

57. West, J. A. et al. The Long Noncoding RNAs NEAT1 and MALAT1 Bind Active Chromatin Sites. Mol. Cell (2014). doi:10.1016/j.molcel.2014.07.012

58. Engreitz, J. M. et al. RNA-RNA interactions enable specific targeting of noncoding RNAs to nascent pre-mRNAs and chromatin sites. Cell 159, 188-199 (2014). 
59. Schoeftner, S. \& Blasco, M. A. Developmentally regulated transcription of mammalian telomeres by DNA-dependent RNA polymerase II. Nat. Cell Biol. (2008). doi:10.1038/ncb1685

60. Mumbach, M. R. et al. HiChIRP reveals RNA-associated chromosome conformation. Nat. Methods (2019). doi:10.1038/s41592-019-0407-x

61. Decker, C. J. \& Parker, R. P-bodies and stress granules: possible roles in the control of translation and mRNA degradation. Cold Spring Harbor perspectives in biology 4, (2012).

62. Wolozin, B. \& Ivanov, P. Stress granules and neurodegeneration. Nature Reviews Neuroscience (2019). doi:10.1038/s41583-019-0222-5

63. Banani, S. F., Lee, H. O., Hyman, A. A. \& Rosen, M. K. Biomolecular condensates: Organizers of cellular biochemistry. Nature Reviews Molecular Cell Biology (2017). doi:10.1038/nrm.2017.7

64. Goldfarb, K. C. \& Cech, T. R. Targeted CRISPR disruption reveals a role for RNase MRP RNA in human preribosomal RNA processing. Genes Dev. (2017). doi:10.1101/gad.286963.116

65. Dragon, F. et al. A large nucleolar U3 ribonucleoprotein required for $18 \mathrm{~S}$ ribosomal RNA biogenesis. Nature (2002). doi:10.1038/nature00769

66. Baßler, J. \& Hurt, E. Eukaryotic Ribosome Assembly. Annu. Rev. Biochem. (2019). doi:10.1146/annurev-biochem-013118-110817

67. Lee, Y. \& Rio, D. C. Mechanisms and Regulation of Alternative Pre-mRNA Splicing. Annu. Rev. Biochem. (2015). doi:10.1146/annurev-biochem-060614-034316

68. Bentley, D. L. Rules of engagement: Co-transcriptional recruitment of pre-mRNA processing factors. Current Opinion in Cell Biology (2005). doi:10.1016/j.ceb.2005.04.006

69. Neugebauer, K. M. On the importance of being co-transcriptional. J. Cell Sci. (2002). doi: $10.1242 /$ jes. 00073

70. Bentley, D. L. Coupling mRNA processing with transcription in time and space. Nature Reviews Genetics 15, 163-175 (2014). 
1514 71. McCracken, S. et al. The C-terminal domain of RNA polymerase II couples mRNA processing to transcription. Nature (1997). doi:10.1038/385357a0

72. Martin, R. M., Rino, J., Carvalho, C., Kirchhausen, T. \& Carmo-Fonseca, M. Live-Cell Visualization of Pre-mRNA Splicing with Single-Molecule Sensitivity. Cell Rep. (2013).

73. Herzel, L., Ottoz, D. S. M., Alpert, T. \& Neugebauer, K. M. Splicing and transcription touch 1520 base: Co-transcriptional spliceosome assembly and function. Nature Reviews Molecular Cell Biology (2017). doi:10.1038/nrm.2017.63

74. Pandya-Jones, A. \& Black, D. L. Co-transcriptional splicing of constitutive and alternative exons. RNA (2009). doi:10.1261/rna.1714509

75. Calvet, J. P. \& Pederson, T. Base-pairing interactions between small nuclear RNAs and nuclear RNA precursors as revealed by psoralen cross-linking in vivo. Cell (1981). doi:10.1016/00928674(81)90205-1

76. Karijolich, J. \& Yu, Y. T. Spliceosomal snRNA modifications and their function. RNA Biology 1528 (2010). doi:10.4161/rna.7.2.11207

77. Maden, B. E. H. The Numerous Modified Nucleotides in Eukaryotic Ribosomal RNA. Prog. Nucleic Acid Res. Mol. Biol. (1990). doi:10.1016/S0079-6603(08)60629-7

78. Reddy, R. \& Busch, H. Small Nuclear RNAs: RNA Sequences, Structure, and Modifications. in Structure and Function of Major and Minor Small Nuclear Ribonucleoprotein Particles (1988). doi:10.1007/978-3-642-73020-7_1

79. Tycowski, K. T., You, Z. H., Graham, P. J. \& Steitz, J. A. Modification of U6 spliceosomal RNA 1535

80. Nizami, Z., Deryusheva, S. \& Gall, J. G. The Cajal body and histone locus body. Cold Spring Harbor perspectives in biology (2010). doi:10.1101/cshperspect.a000653 
pseudouridylation guide RNAs. EMBO J. (2002). doi:10.1093/emboj/21.11.2746

1540 82. Richard, P. et al. A common sequence motif determines the Cajal body-specific localization of box H/ACA scaRNAs. EMBO J. (2003). doi:10.1093/emboj/cdg394

83. Machyna, M. et al. The coilin interactome identifies hundreds of small noncoding RNAs that traffic through cajal bodies. Mol. Cell (2014). doi:10.1016/j.molcel.2014.10.004

84. Machyna, M., Neugebauer, K. M. \& Staněk, D. Coilin: The first 25 years. RNA Biol. (2015). doi:10.1080/15476286.2015.1034923

85. Machyna, M., Heyn, P. \& Neugebauer, K. M. Cajal bodies: Where form meets function. Wiley Interdisciplinary Reviews: RNA (2013). doi:10.1002/wrna.1139

86. Deryusheva, S. \& Gall, J. G. Small Cajal body-specific RNAs of Drosophila function in the absence of Cajal bodies. Mol. Biol. Cell (2009). doi:10.1091/mbc.E09-09-0777

1550 87. Smith, K. P., Carter, K. C., Johnson, C. V. \& Lawrence, J. B. U2 and U1 snRNA gene loci associate with coiled bodies. J. Cell. Biochem. (1995). doi:10.1002/jcb.240590408

88. Marzluff, W. F., Wagner, E. J. \& Duronio, R. J. Metabolism and regulation of canonical histone mRNAs: Life without a poly(A) tail. Nature Reviews Genetics (2008). doi:10.1038/nrg2438

89. Shevtsov, S. P. \& Dundr, M. Nucleation of nuclear bodies by RNA. Nat. Cell Biol. 13, 167-173 (2011).

90. Kaiser, T. E., Intine, R. V. \& Dundr, M. De novo formation of a subnuclear body. Science (80-. ). (2008). doi:10.1126/science. 1165216

1558 91. Maison, C. et al. Higher-order structure in pericentric heterochromatin involves a distinct pattern of histone modification and an RNA component. Nat. Genet. (2002). doi:10.1038/ng843

1560 92. Maison, C. \& Almouzni, G. HP1 and the dynamics of heterochromatin maintenance. Nature 1561 Reviews Molecular Cell Biology (2004). doi:10.1038/nrm1355

1562 93. Barutcu, A. R., Blencowe, B. J. \& Rinn, J. L. Differential contribution of steady-state RNA and 
active transcription in chromatin organization . EMBO Rep. (2019). doi:10.15252/embr.201948068

94. Probst, A. V. et al. A Strand-specific burst in transcription of pericentric satellites is required for chromocenter formation and early mouse development. Dev. Cell (2010). doi:10.1016/j.devcel.2010.09.002

95. Casanova, M. et al. Heterochromatin Reorganization during Early Mouse Development Requires a Single-Stranded Noncoding Transcript. Cell Rep. (2013). doi:10.1016/j.celrep.2013.08.015

96. Santenard, A. et al. Heterochromatin formation in the mouse embryo requires critical residues of the histone variant H3.3. Nat. Cell Biol. (2010). doi:10.1038/ncb2089

97. Lee, S. et al. Noncoding RNA NORAD Regulates Genomic Stability by Sequestering PUMILIO Proteins. Cell (2016). doi:10.1016/j.cell.2015.12.017

98. Chao, S. H. \& Price, D. H. Flavopiridol Inactivates P-TEFb and Blocks Most RNA Polymerase II Transcription in Vivo. J. Biol. Chem. (2001). doi:10.1074/jbc.M102306200

99. Jonkers, I., Kwak, H. \& Lis, J. T. Genome-wide dynamics of Pol II elongation and its interplay with promoter proximal pausing, chromatin, and exons. Elife 2014, (2014).

100. Clark, M. B. et al. Genome-wide analysis of long noncoding RNA stability. Genome Res. (2012). doi:10.1101/gr.131037.111

101. Chu, C. et al. Systematic discovery of Xist RNA binding proteins. Cell 161, 404-416 (2015).

102. Lu, Z. et al. RNA Duplex Map in Living Cells Reveals Higher-Order Transcriptome Structure. Cell (2016). doi:10.1016/j.cell.2016.04.028

103. McHugh, C. A. et al. The Xist lncRNA interacts directly with SHARP to silence transcription through HDAC3. Nature 521, 232-236 (2015).

104. Chen, C.-K. et al. Xist recruits the X chromosome to the nuclear lamina to enable chromosomewide silencing. Science (80-. ). 354, 468-472 (2016). 
1587 105. Cirillo, D. et al. Quantitative predictions of protein interactions with long noncoding RNAs: To the Editor. Nature Methods (2016). doi:10.1038/nmeth.4100

1589 106. Dossin, F. et al. SPEN integrates transcriptional and epigenetic control of X-inactivation. Nature (2020). doi:10.1038/s41586-020-1974-9

107. Żylicz, J. J. et al. The Implication of Early Chromatin Changes in X Chromosome Inactivation. Cell (2019). doi:10.1016/j.cell.2018.11.041

108. Kanduri, C. Kenq1ot1: A chromatin regulatory RNA. Seminars in Cell and Developmental Biology (2011). doi:10.1016/j.semcdb.2011.02.020

109. Nagano, T. \& Fraser, P. Emerging similarities in epigenetic gene silencing by long noncoding RNAs. Mammalian Genome (2009). doi:10.1007/s00335-009-9218-1

110. Stricker, S. H. et al. Silencing and transcriptional properties of the imprinted Airn ncRNA are independent of the endogenous promoter. EMBO J. (2008). doi:10.1038/emboj.2008.239

111. Braidotti, G. et al. The Air noncoding RNA: An imprinted cis-silencing transcript. in Cold Spring Harbor Symposia on Quantitative Biology (2004). doi:10.1101/sqb.2004.69.55

112. Sleutels, F., Tjon, G., Ludwig, T. \& Barlow, D. P. Imprinted silencing of Slc22a2 and Slc22a3 does not need transcriptional overlap between Igf2r and Air. EMBO J. (2003). doi:10.1093/emboj/cdg341

113. Olivero, C. E. et al. p53 Activates the Long Noncoding RNA Pvt1b to Inhibit Myc and Suppress Tumorigenesis. Mol. Cell (2020). doi:10.1016/j.molcel.2019.12.014

114. Rom, A. et al. Regulation of CHD2 expression by the Chaserr long noncoding RNA gene is essential for viability. Nat. Commun. (2019). doi:10.1038/s41467-019-13075-8

115. Engreitz, J. M. et al. Local regulation of gene expression by lncRNA promoters, transcription and splicing. Nature (2016). doi:10.1038/nature20149 
1612 117. Hnisz, D., Shrinivas, K., Young, R. A., Chakraborty, A. K. \& Sharp, P. A. A Phase Separation Model for Transcriptional Control. Cell (2017). doi:10.1016/j.cell.2017.02.007

1614 118. Santoro, F. et al. Imprinted Igf2r silencing depends on continuous airn lncRNA expression and is 1615 not restricted to a developmental window. Dev. (2013). doi:10.1242/dev.088849

1616 1617

119. Gall, J. G. Cajal Bodies: The First 100 Years. Annu. Rev. Cell Dev. Biol. (2000). doi:10.1146/annurev.cellbio.16.1.273

120. Jády, B. E. \& Kiss, T. A small nucleolar guide RNA functions both in 2'-O-ribose methylation and pseudouridylation of the U5 spliceosomal RNA. EMBO J. (2001). doi:10.1093/emboj/20.3.541

121. Ogg, S. C. \& Lamond, A. I. Cajal bodies and coilin - Moving towards function. Journal of Cell Biology 159, 17-21 (2002).

122. Matera, A. G. \& Frey, M. R. Coiled bodies and gems: Janus or gemini? Am. J. Hum. Genet. (1998). doi:10.1086/301992

123. Tucker, K. E. et al. Residual Cajal bodies in coilin knockout mice fail to recruit Sm snRNPs and SMN, the spinal muscular atrophy gene product. J. Cell Biol. (2001). doi:10.1083/jcb.200104083

124. Nizami, Z., Deryusheva, S. \& Gall, J. G. The Cajal body and histone locus body. Cold Spring Harbor perspectives in biology 2, (2010).

125. Shishkin, A. A. et al. Simultaneous generation of many RNA-seq libraries in a single reaction. Nat. Methods 12, 323-325 (2015).

126. Martin, M. Cutadapt removes adapter sequences from high-throughput sequencing reads. EMBnet.journal (2011). doi:10.14806/ej.17.1.200

127. Kim, D., Langmead, B. \& Salzberg, S. L. HISAT: A fast spliced aligner with low memory requirements. Nat. Methods (2015). doi:10.1038/nmeth.3317

128. Jin, Y., Tam, O. H., Paniagua, E. \& Hammell, M. TEtranscripts: A package for including transposable elements in differential expression analysis of RNA-seq datasets. Bioinformatics 
(2015). doi:10.1093/bioinformatics/btv422

1638 129. Langmead, B. \& Salzberg, S. L. Fast gapped-read alignment with Bowtie 2. Nat. Methods 9, 357$1639359(2012)$.

1640 130. Krueger, F. \& Andrews, S. R. SNPsplit: Allele-specific splitting of alignments between genomes with known SNP genotypes. F1000Research (2016). doi:10.12688/f1000research.9037.1

1642 131. Smit, A., Hubley, R. \& Grenn, P. RepeatMasker Open-4.0. RepeatMasker Open-4.0.7. (2015).

1643 132. Quinlan, A. R. \& Hall, I. M. BEDTools: A flexible suite of utilities for comparing genomic features. Bioinformatics (2010). doi:10.1093/bioinformatics/btq033

1645 133. Ewels, P., Magnusson, M., Lundin, S. \& Käller, M. MultiQC: Summarize analysis results for 1646 multiple tools and samples in a single report. Bioinformatics (2016). doi:10.1093/bioinformatics/btw354

134. Chan, P. P. \& Lowe, T. M. GtRNAdb: A database of transfer RNA genes detected in genomic sequence. Nucleic Acids Res. (2009). doi:10.1093/nar/gkn787

135. Chan, P. P. \& Lowe, T. M. GtRNAdb 2.0: An expanded database of transfer RNA genes identified in complete and draft genomes. Nucleic Acids Res. (2016). doi:10.1093/nar/gkv1309

136. Bolzer, A. et al. Three-dimensional maps of all chromosomes in human male fibroblast nuclei 1653 and prometaphase rosettes. PLoS Biol. (2005). doi:10.1371/journal.pbio.0030157

1654 137. Robinson, J. T. et al. Integrative genomics viewer. Nature Biotechnology (2011). doi:10.1038/nbt.1754

1656 138. Imakaev, M. et al. Iterative correction of Hi-C data reveals hallmarks of chromosome organization. Nat. Methods (2012). doi:10.1038/nmeth.2148 with promoter proximal pausing, chromatin, and exons. Elife (2014). doi:10.7554/eLife.02407 
data. Bioinformatics (2014). doi:10.1093/bioinformatics/btu170

1662 141. Xu, H. et al. FastUniq: A Fast De Novo Duplicates Removal Tool for Paired Short Reads. PLoS 1663 One (2012). doi:10.1371/journal.pone.0052249

1664 142. Lassmann, T., Hayashizaki, Y. \& Daub, C. O. TagDust - A program to eliminate artifacts from 1665 next generation sequencing data. Bioinformatics (2009). doi:10.1093/bioinformatics/btp527

1666 143. Dobin, A. et al. STAR: Ultrafast universal RNA-seq aligner. Bioinformatics (2013). doi:10.1093/bioinformatics/bts635 
Figure 1

A

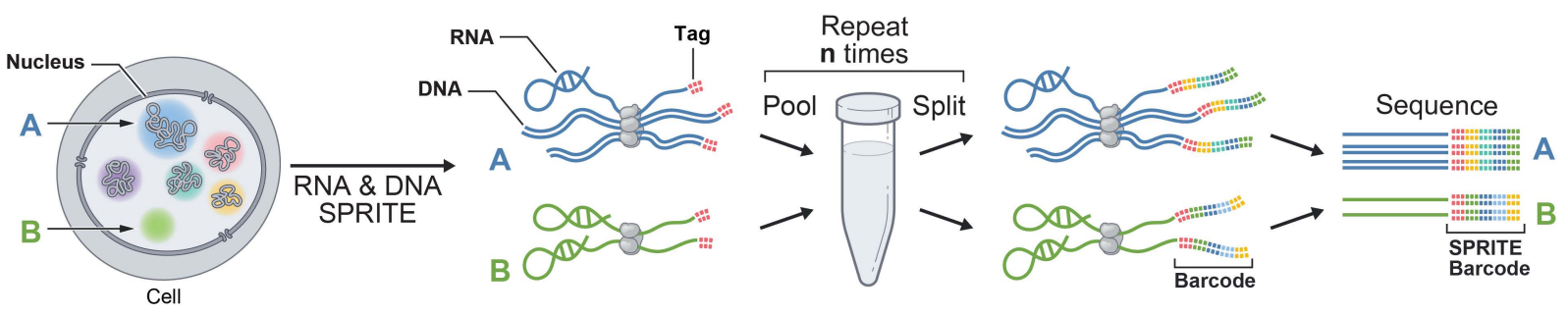

B

RNA-DNA Interactions

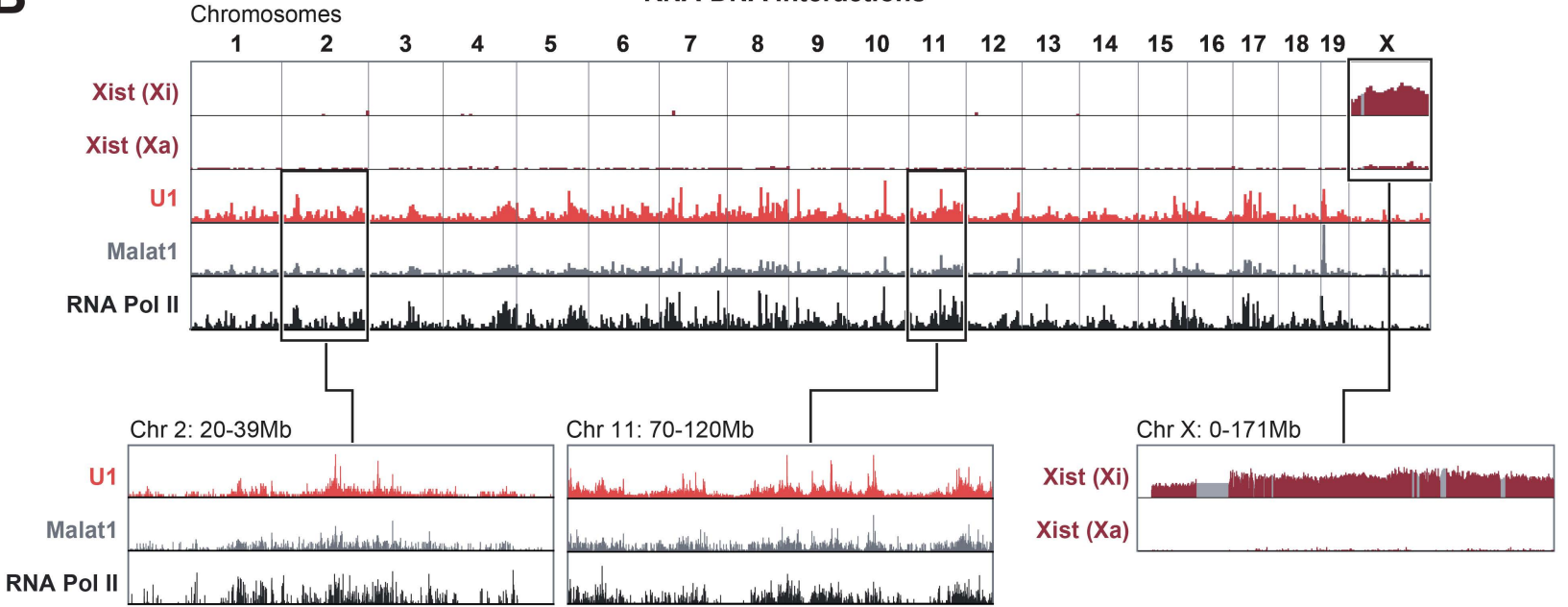


bioRxiv preprint doi: https://doi.org/10.1101/2020.08.25.267435. this version posted August 25, 2020. The copyright holder for this preprint (which was not certified by peer review) is the author/funder. All rights reserved. No reuse allowed without permission.

Figure 2

A

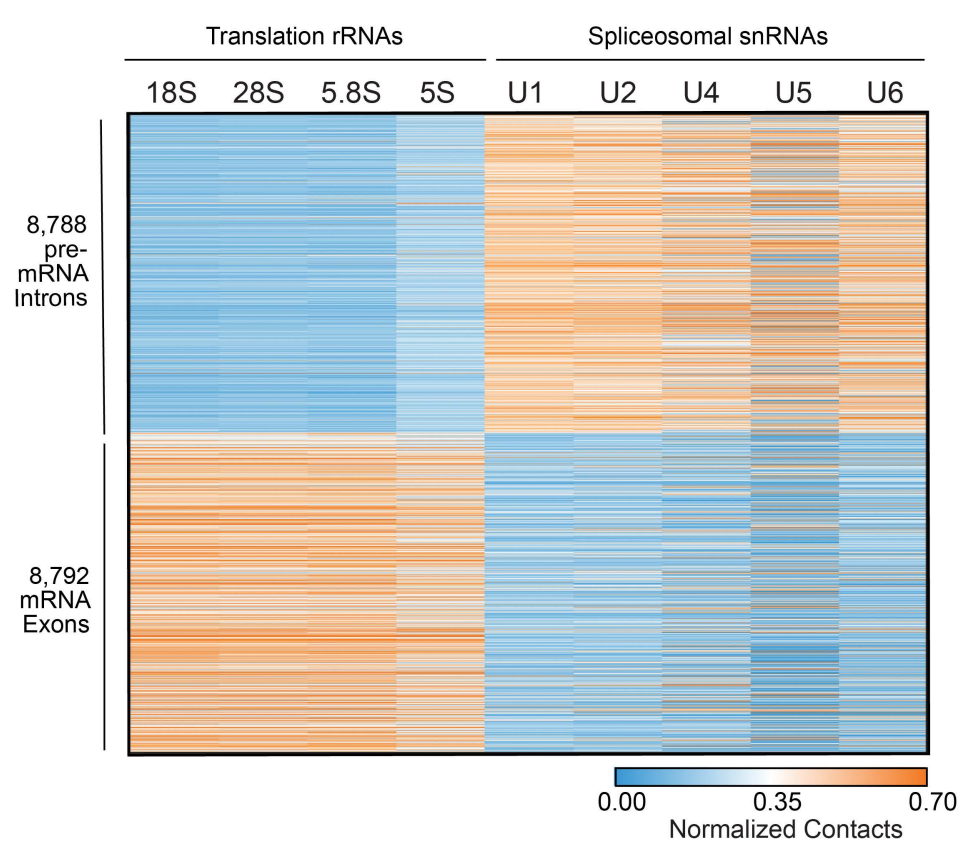

B

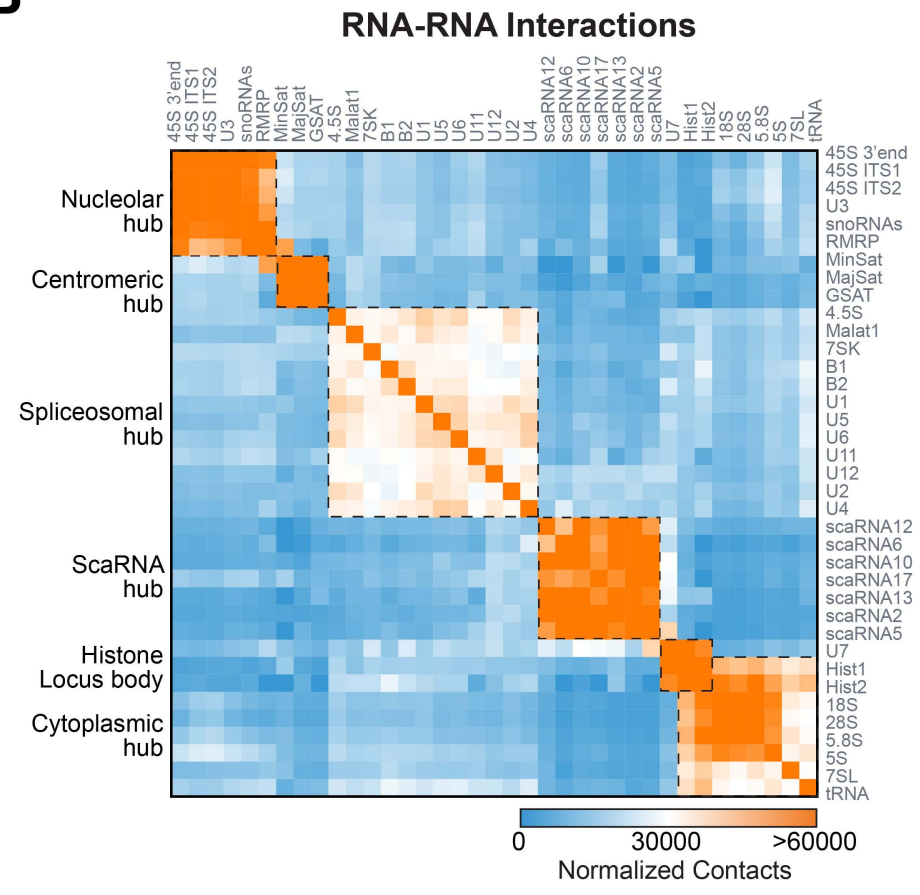


A

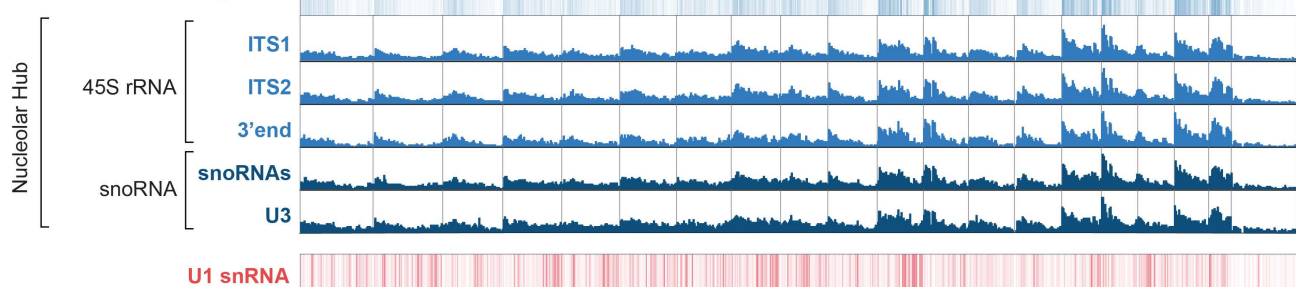

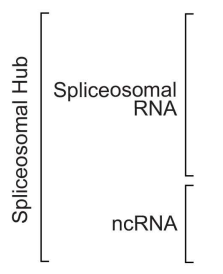

U2

2

$\mathrm{U} 4$

U6

SK

Malat1

RNA Pol II

Gene Density

ע.

\section{C}

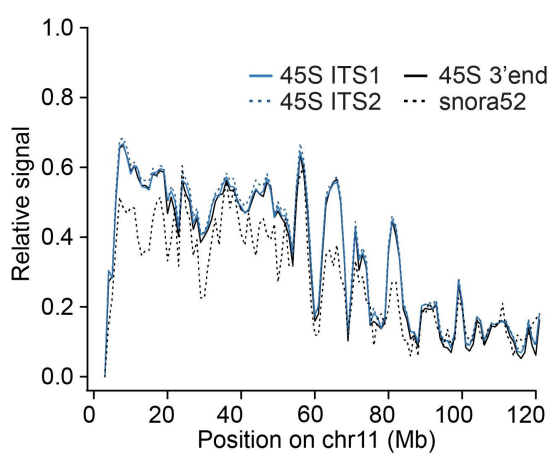

$\mathbf{F}$

RNA-DNA Interactions: Spliceosomal Hub RNAs

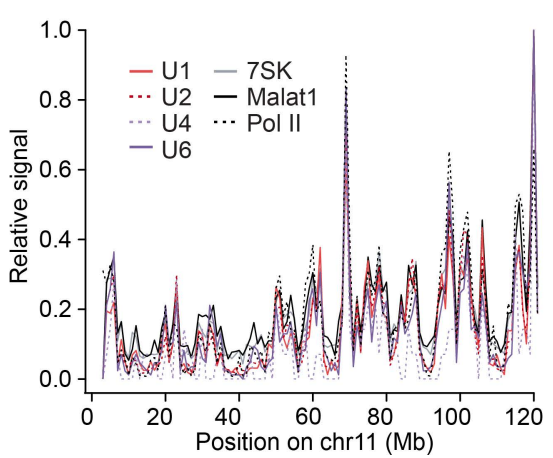

RNA-DNA Interactions rDNA-containing chromosomes

$\begin{array}{llllllllllllll}7 & 8 & 9 & 10 & 11 & 12 & 13 & 14 & 15 & 16 & 17 & 18 & 19 & \mathrm{X}\end{array}$

Figure 3

B
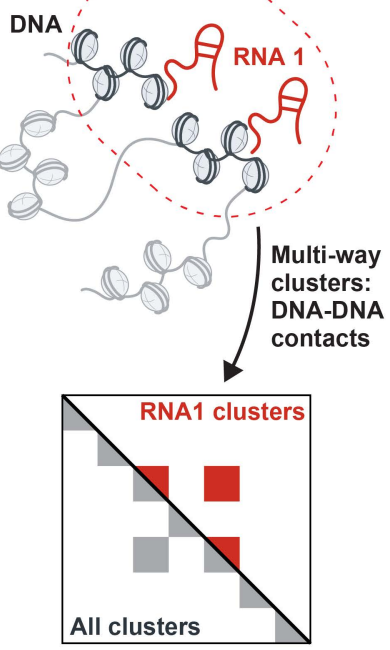

E

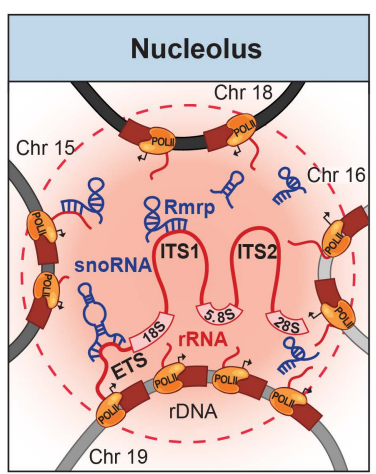

H
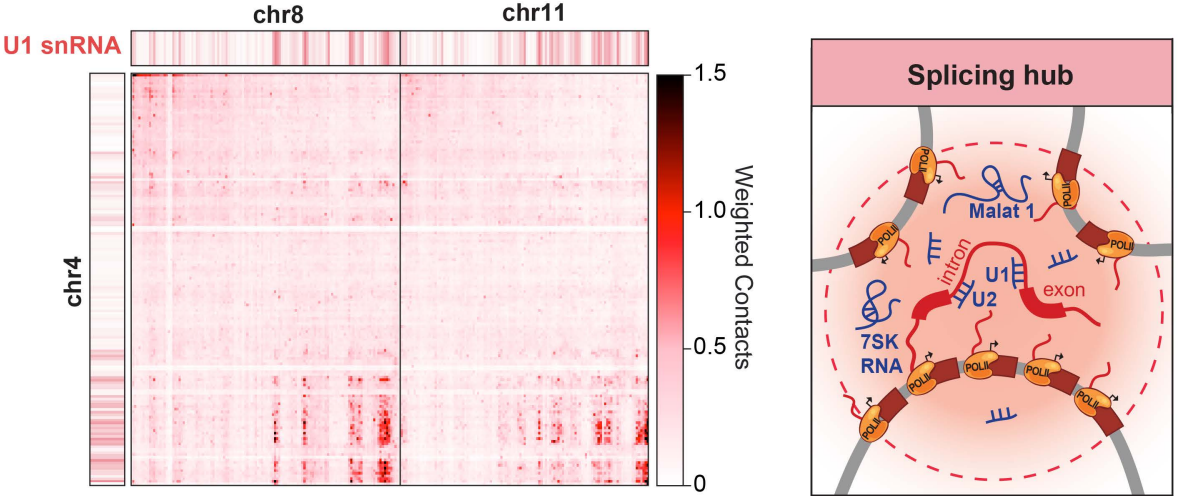
bioRxiv preprint doi: https://doi.org/10.1101/2020.08.25.267435. this version posted August 25, 2020. The copyright holder for this preprint (which was not certified by peer review) is the author/funder. All rights reserved. No reuse allowed without permission.

Figure 4

A
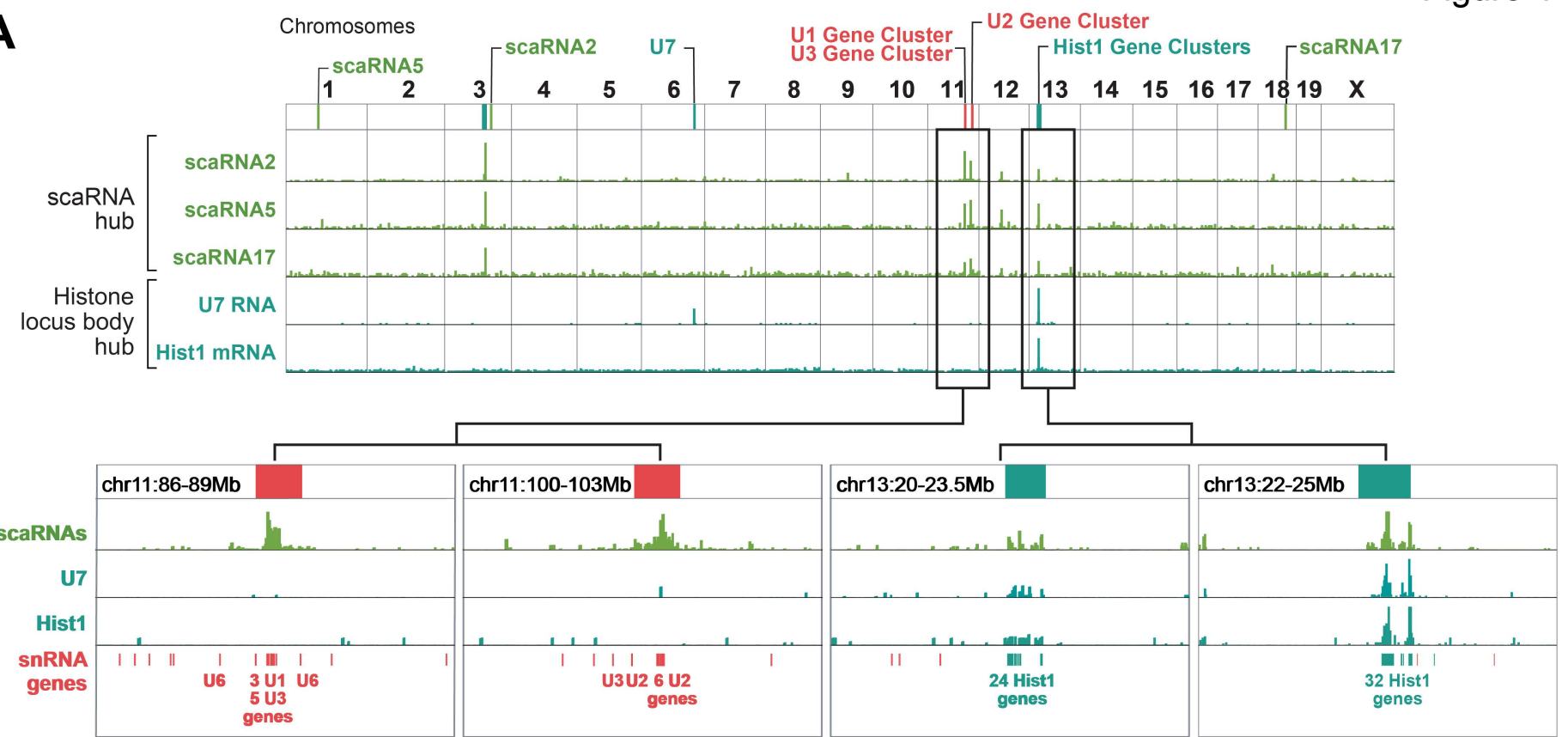

\section{B}

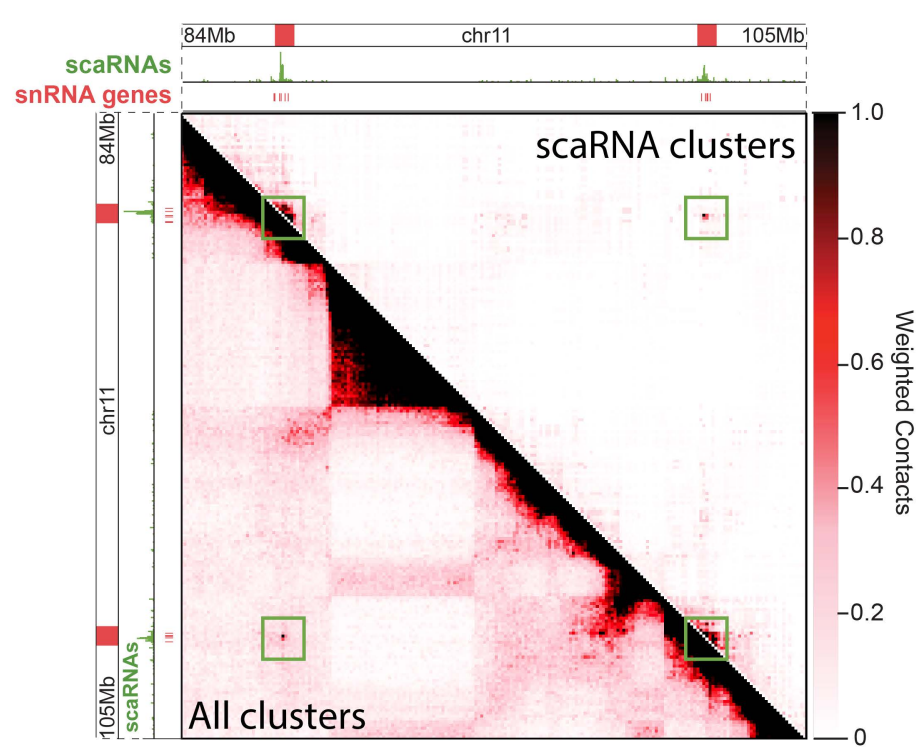

C

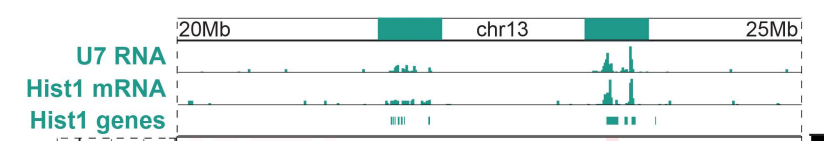

Hist1 genes

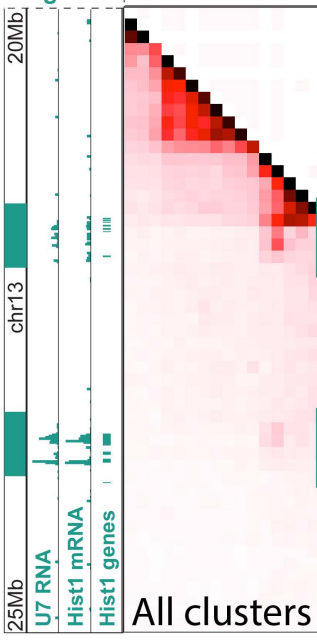

E

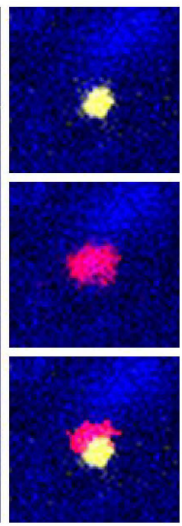

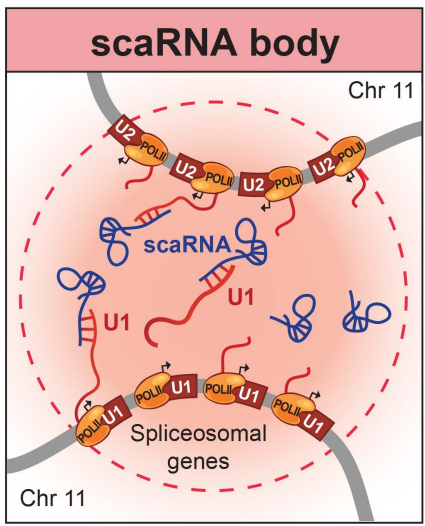

F

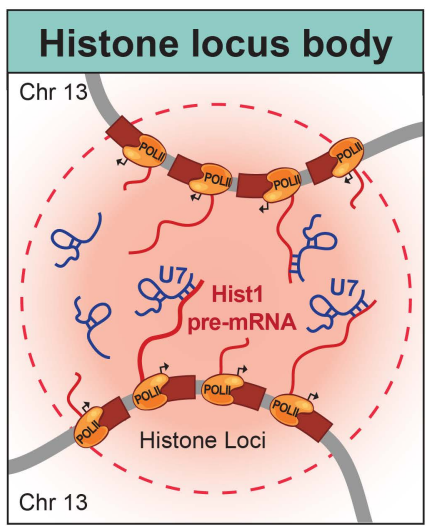


A

Chromosomes

RNA-DNA Interactions

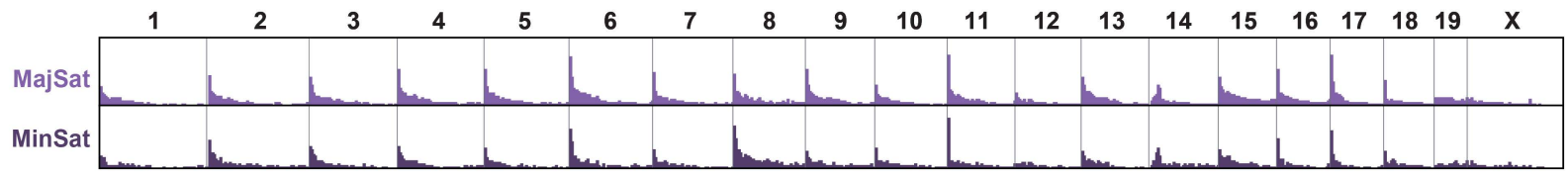

B
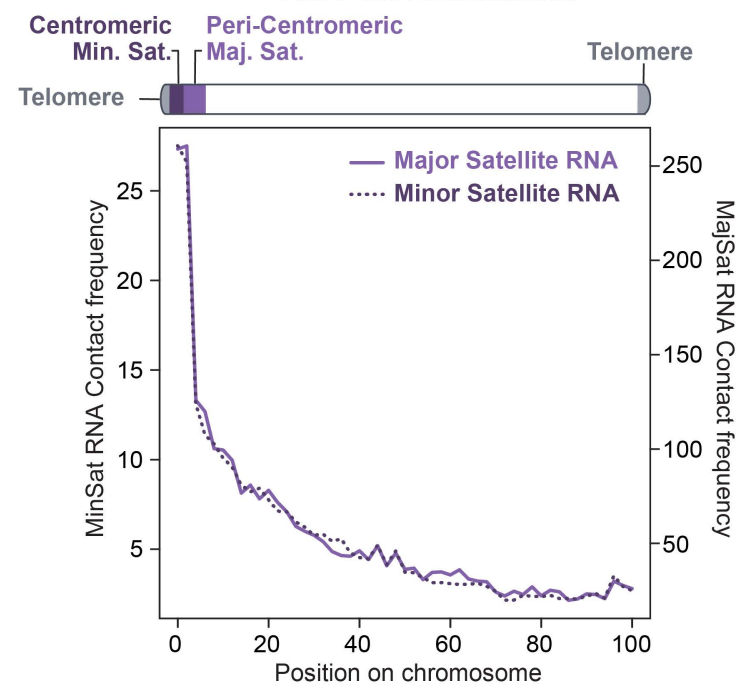

D DNA-FISH $\square$ MinSat $\square$ MajSat

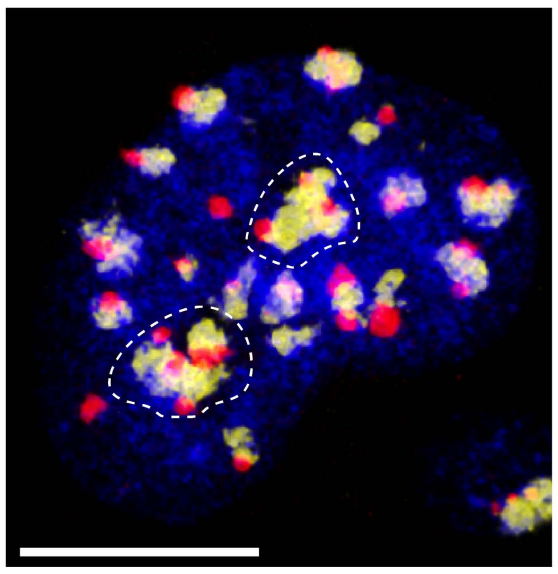

E

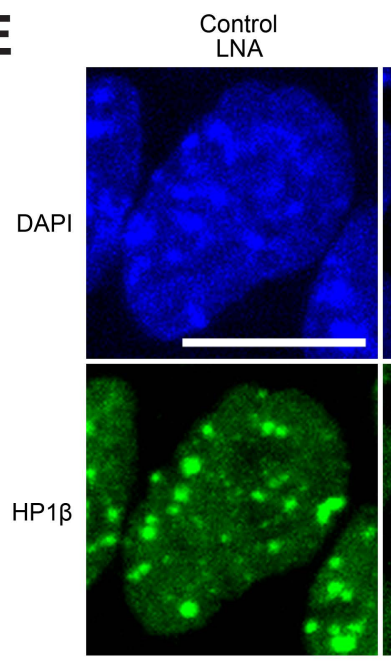

MajSat RNA LNA

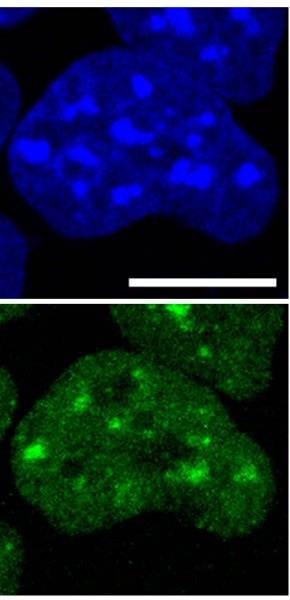

C
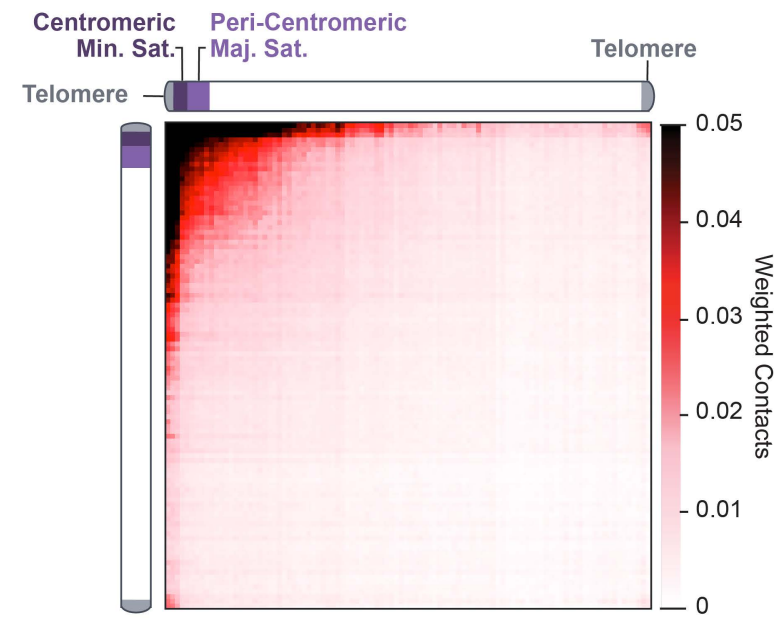

G
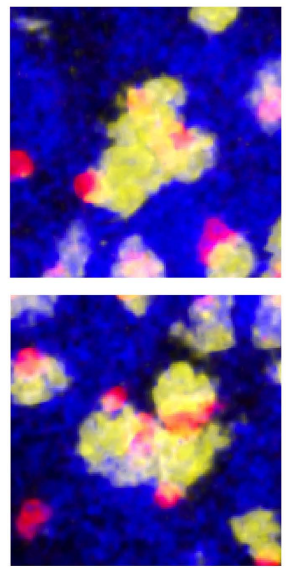

F

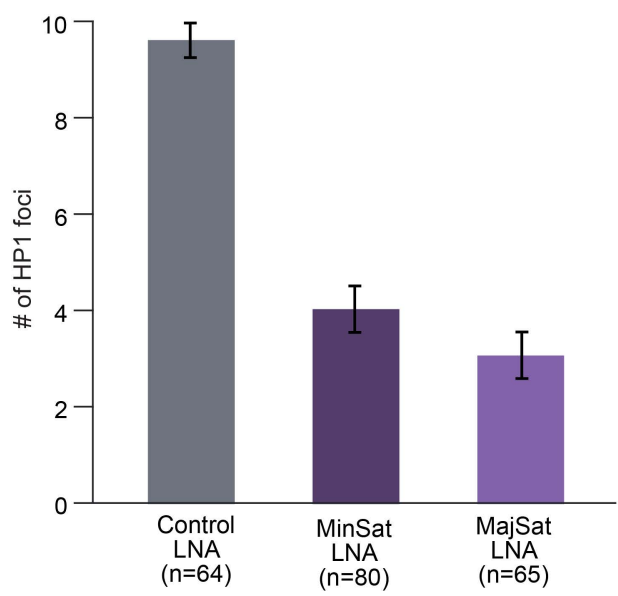


Figure 6

A

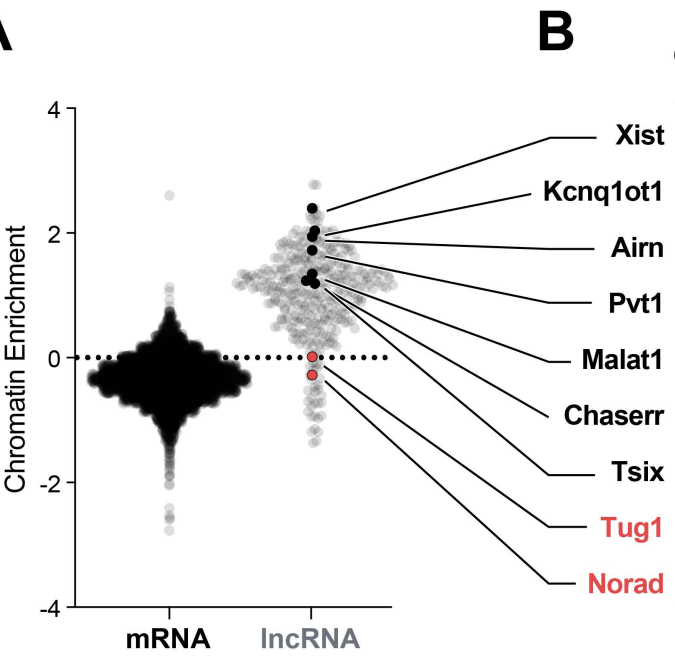

Chromosomes

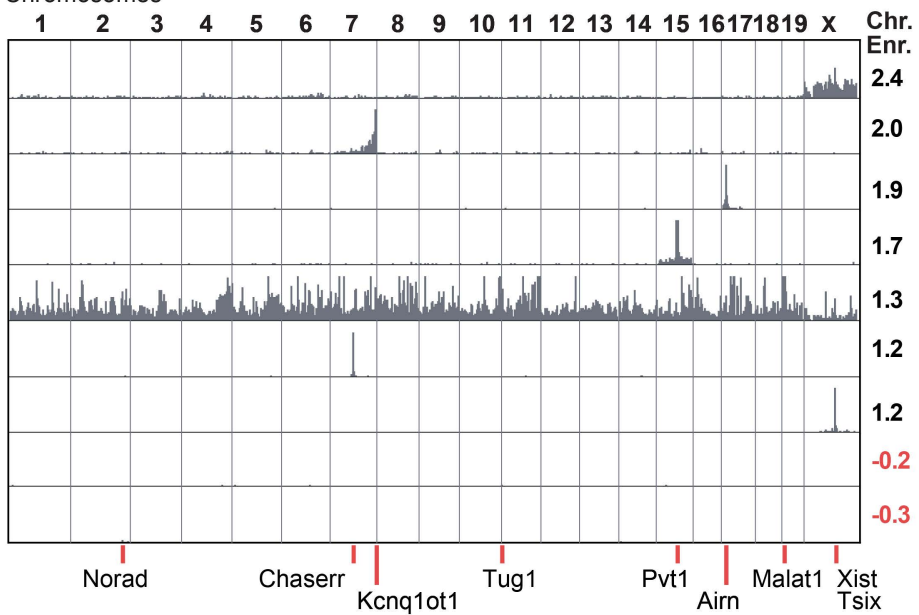

C Chromosomes $\quad$ RNA-DNA Interactions

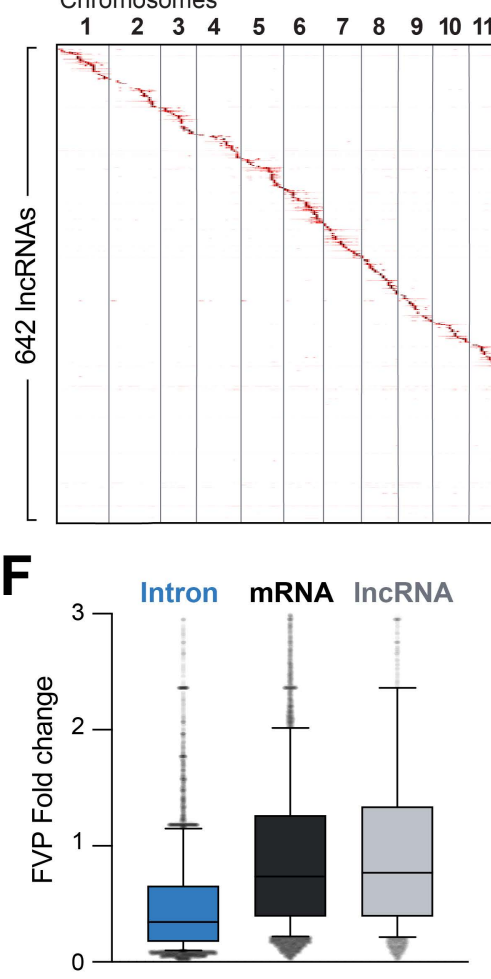

D

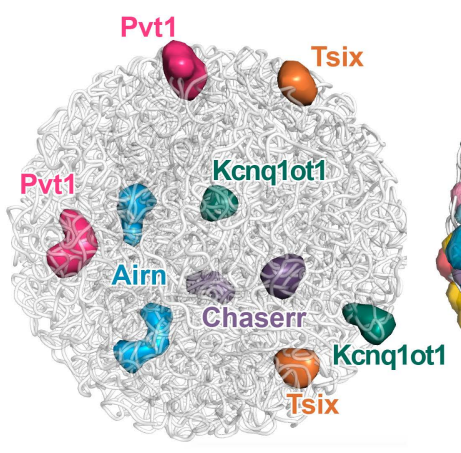

E
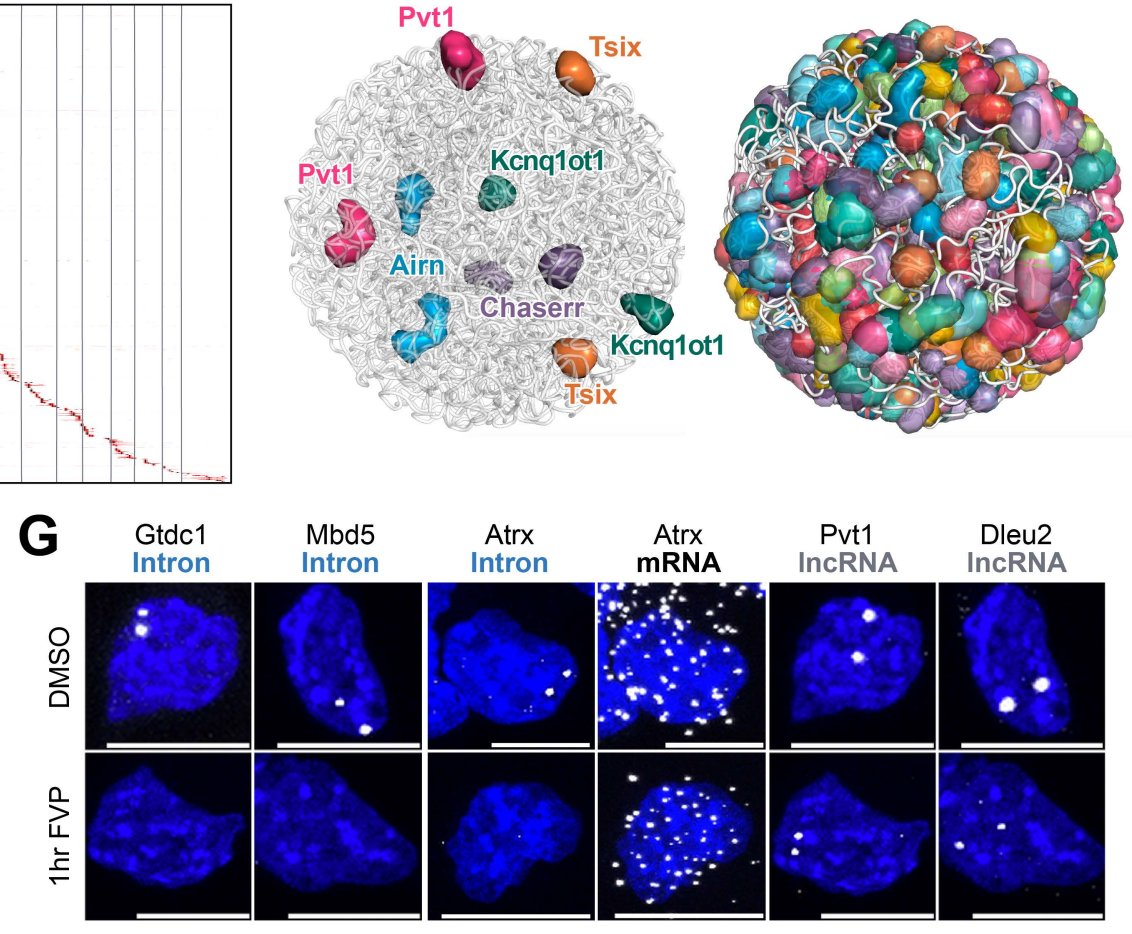

Dleu2 IncRNA

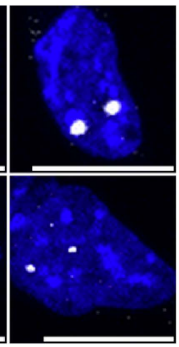


A

\section{FL-SHARP}

$\triangle R R M-S H A R P$

RRM RNA recognition motif SPOC Spen paralogue and orthologue

C

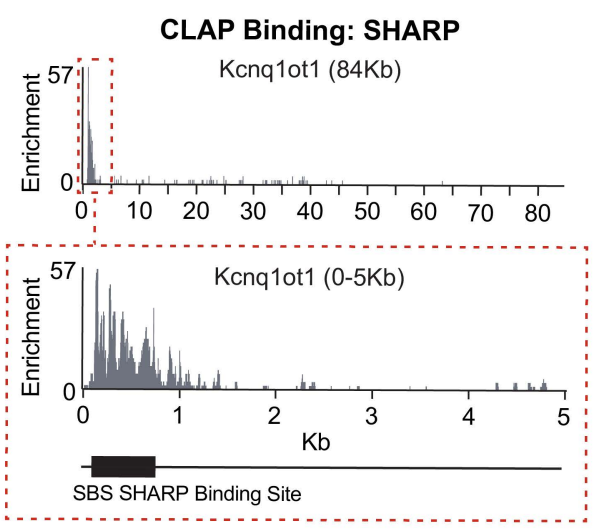

D

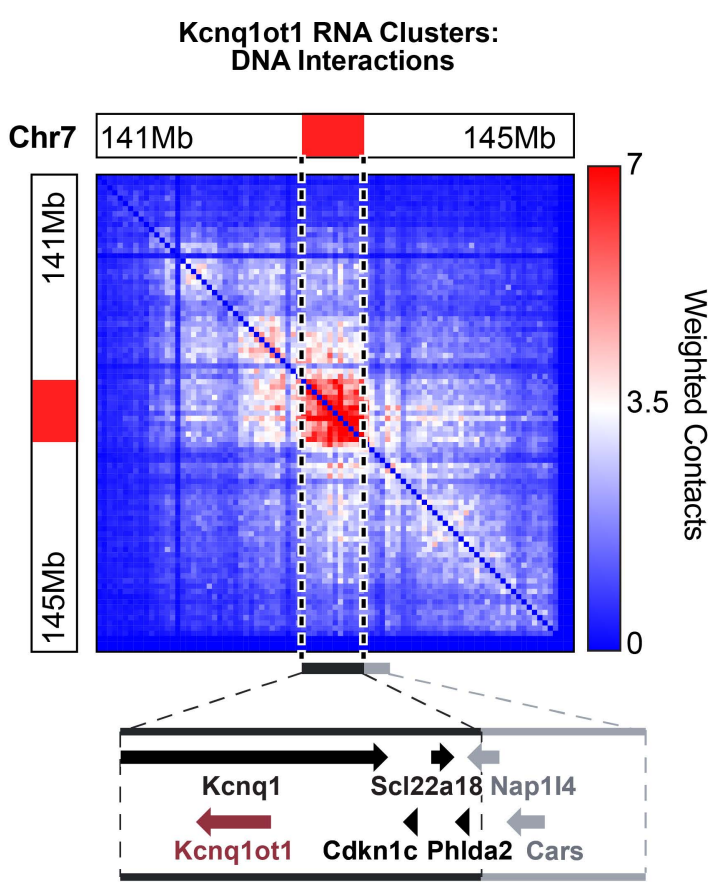

Imprinted genes Non-imprinted genes

B
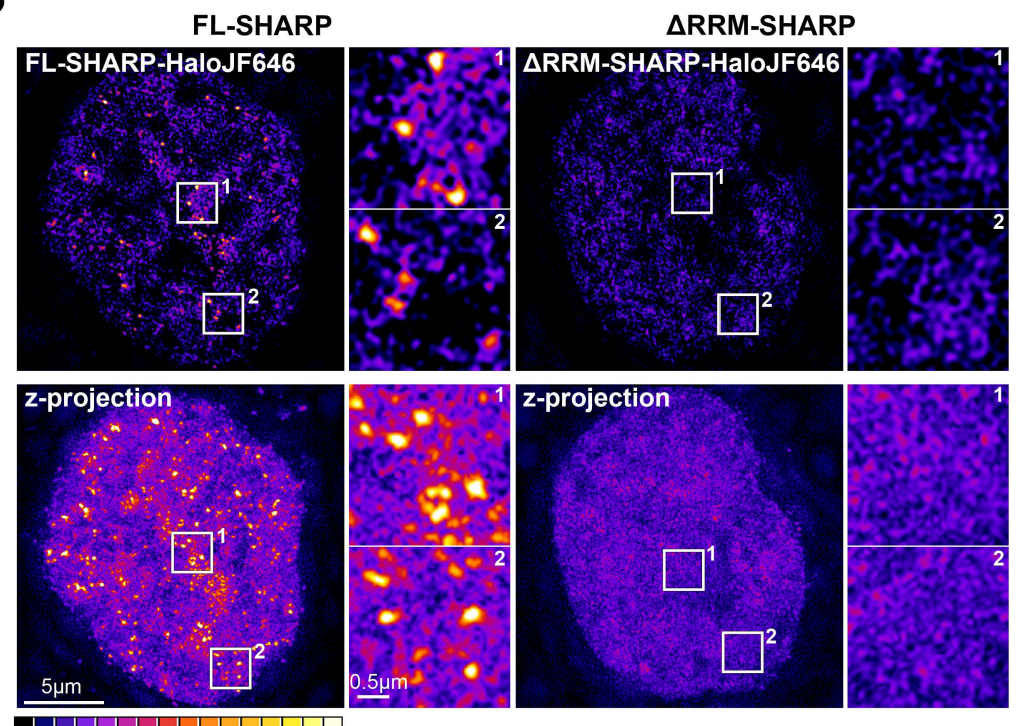

Intensity classification min-max

E

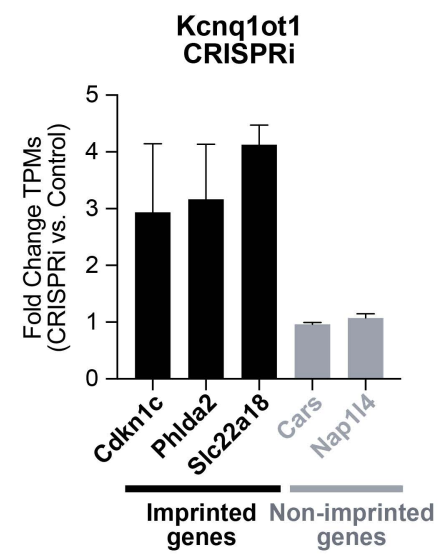

G

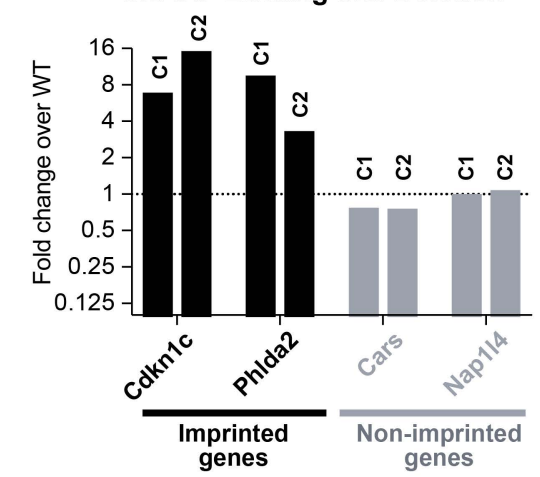

$\mathbf{F}$

Kenq1ot1

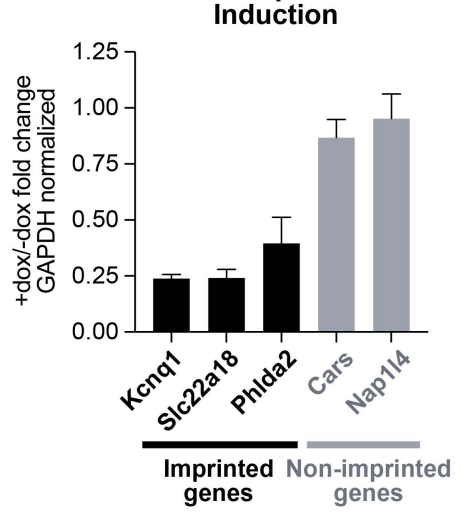

H

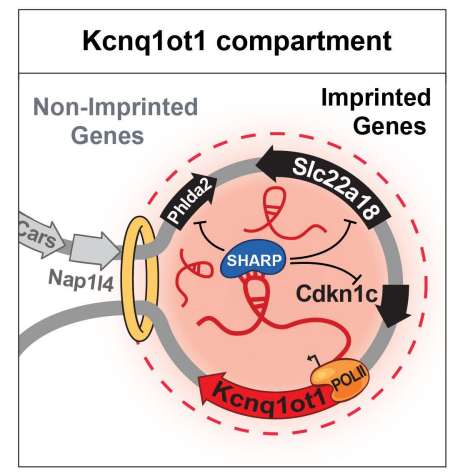


Figure 8
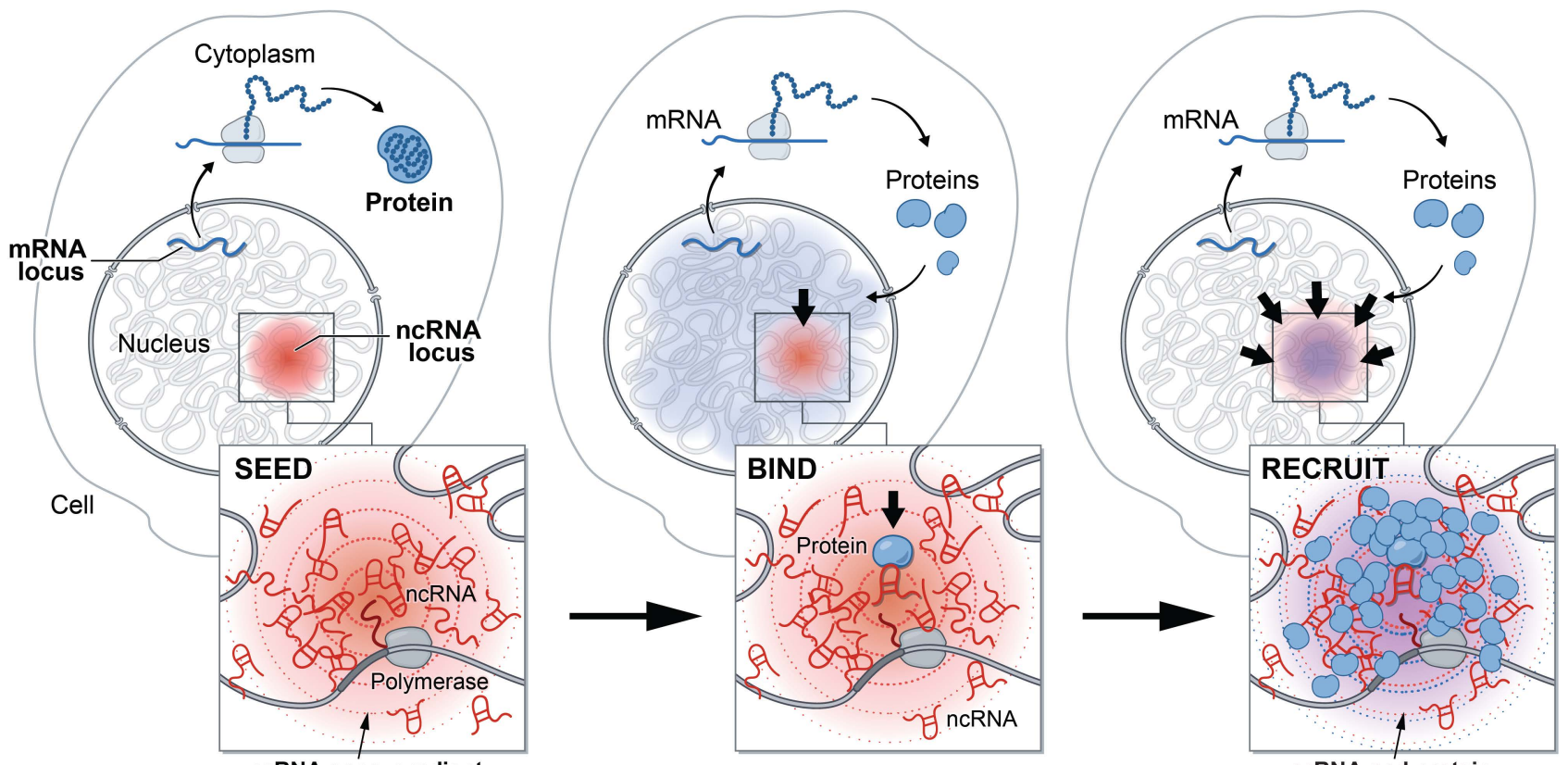

ncRNA conc. gradient

ncRNA and protein conc. compartment 

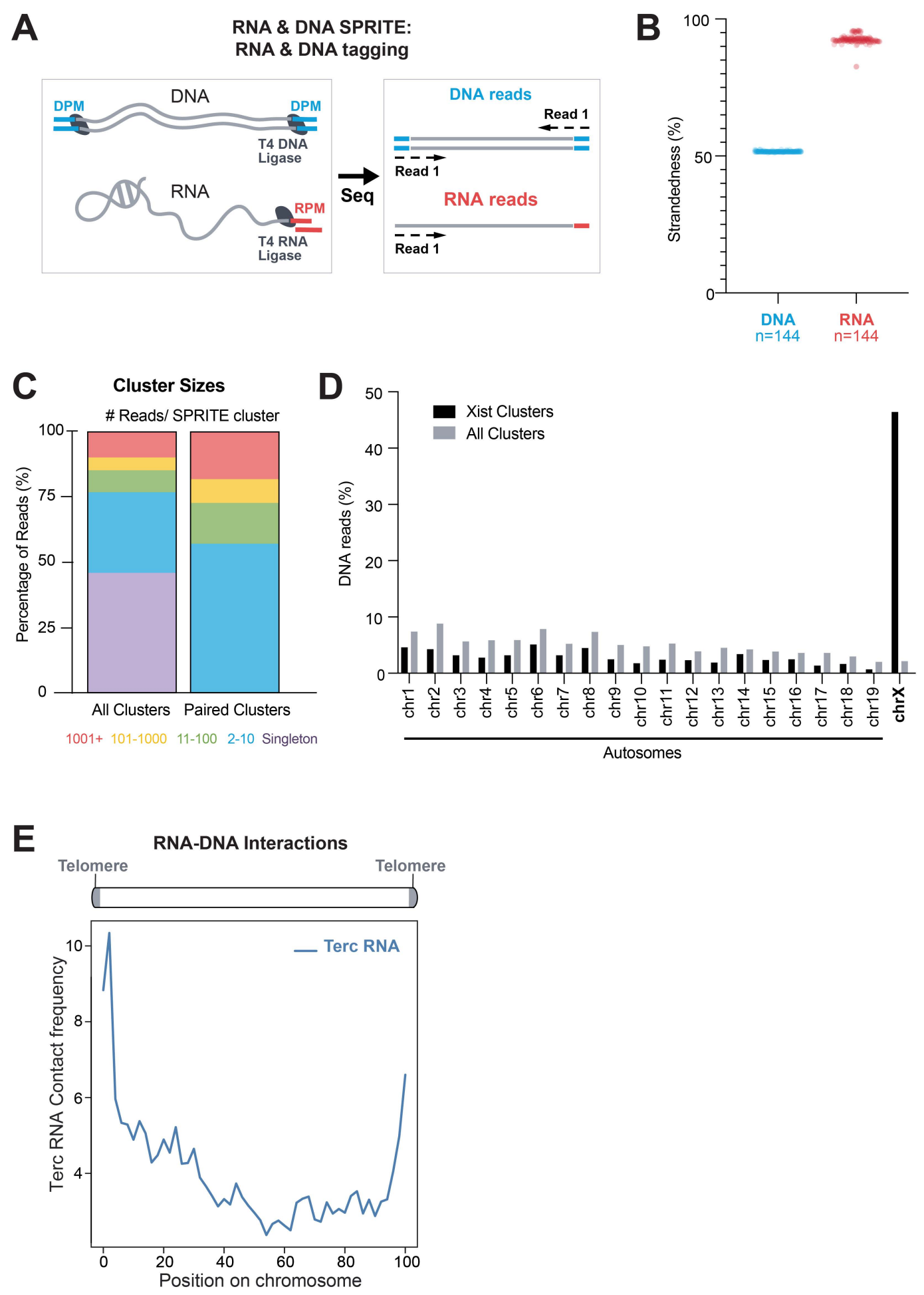
A Chromosomes RNA-DNA Interactions

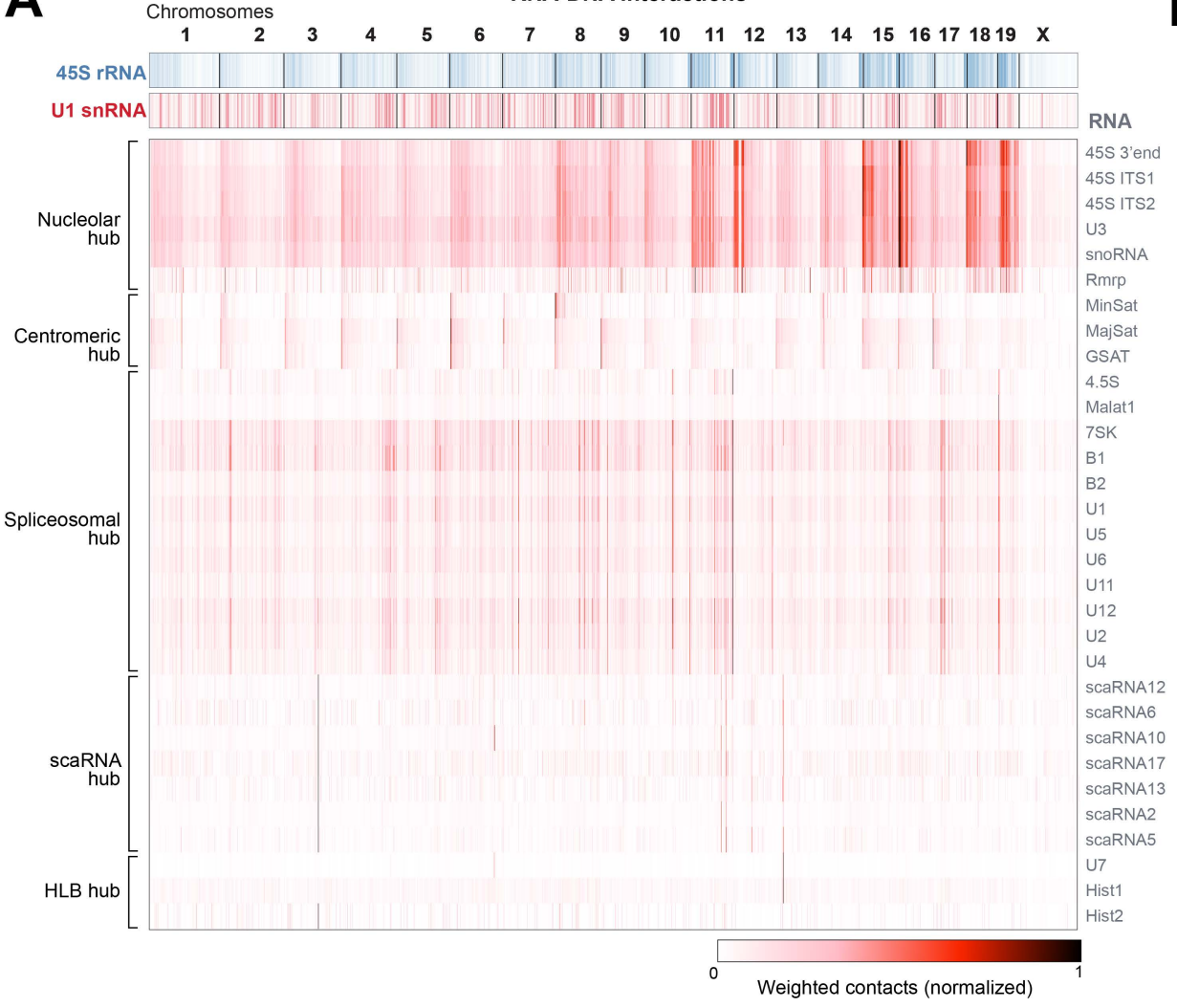

B

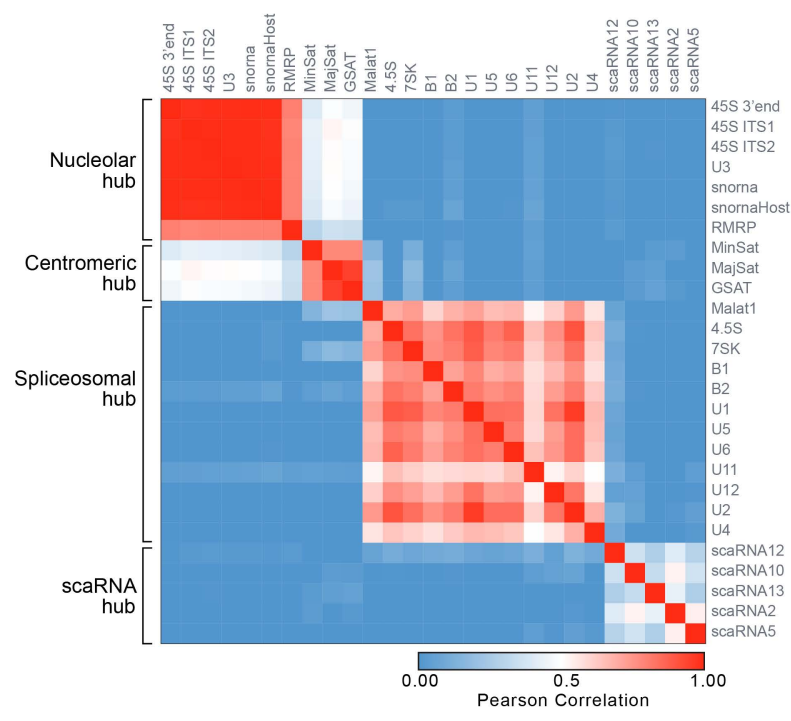

C
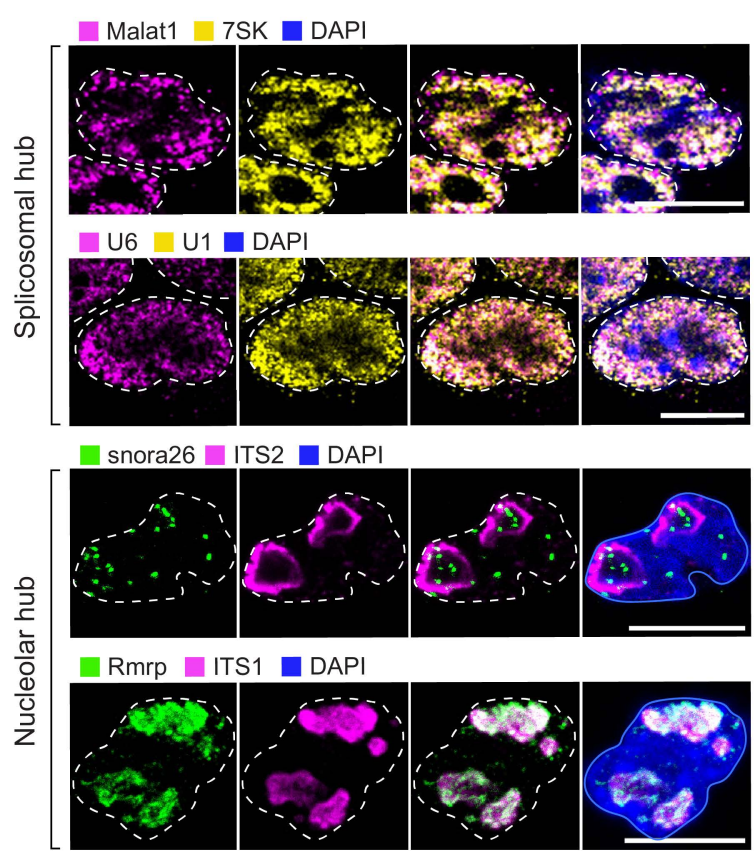

D

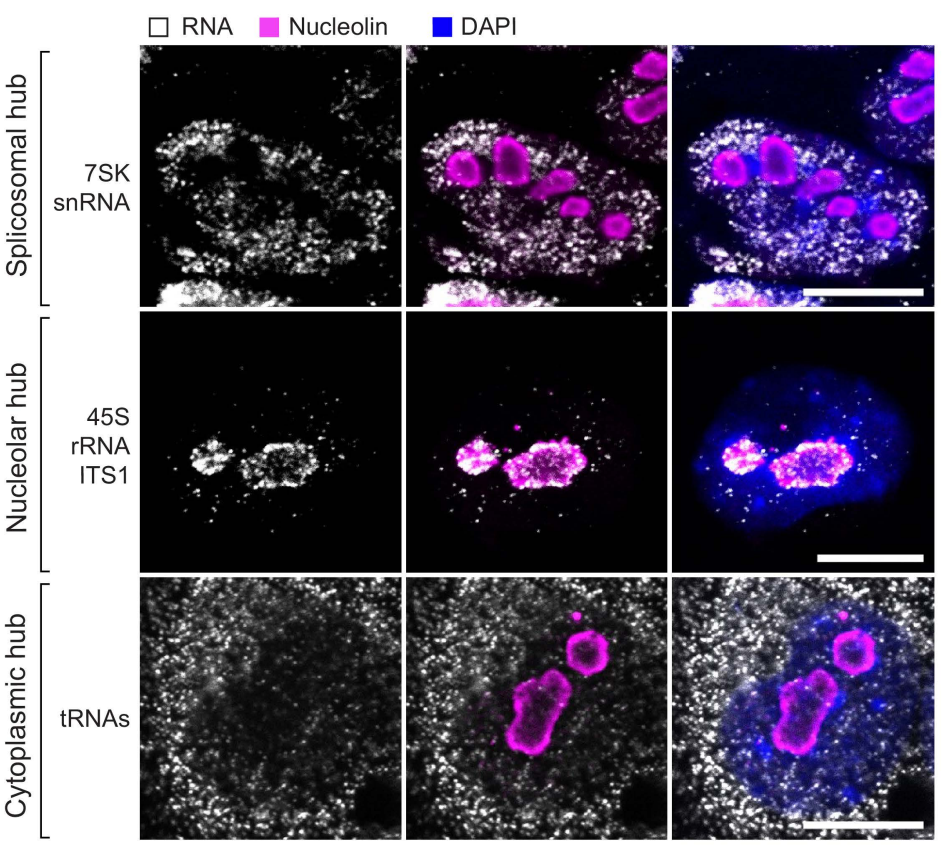


bioRxiv preprint doi: https://doi.org/10.1101/2020.08.25.267435. this version posted August 25, 2020. The copyright holder for this preprint (which was not certified by peer review) is the author/funder. All rights reserved. No reuse allowed without permission.

A snoRNA-DNA Interactions

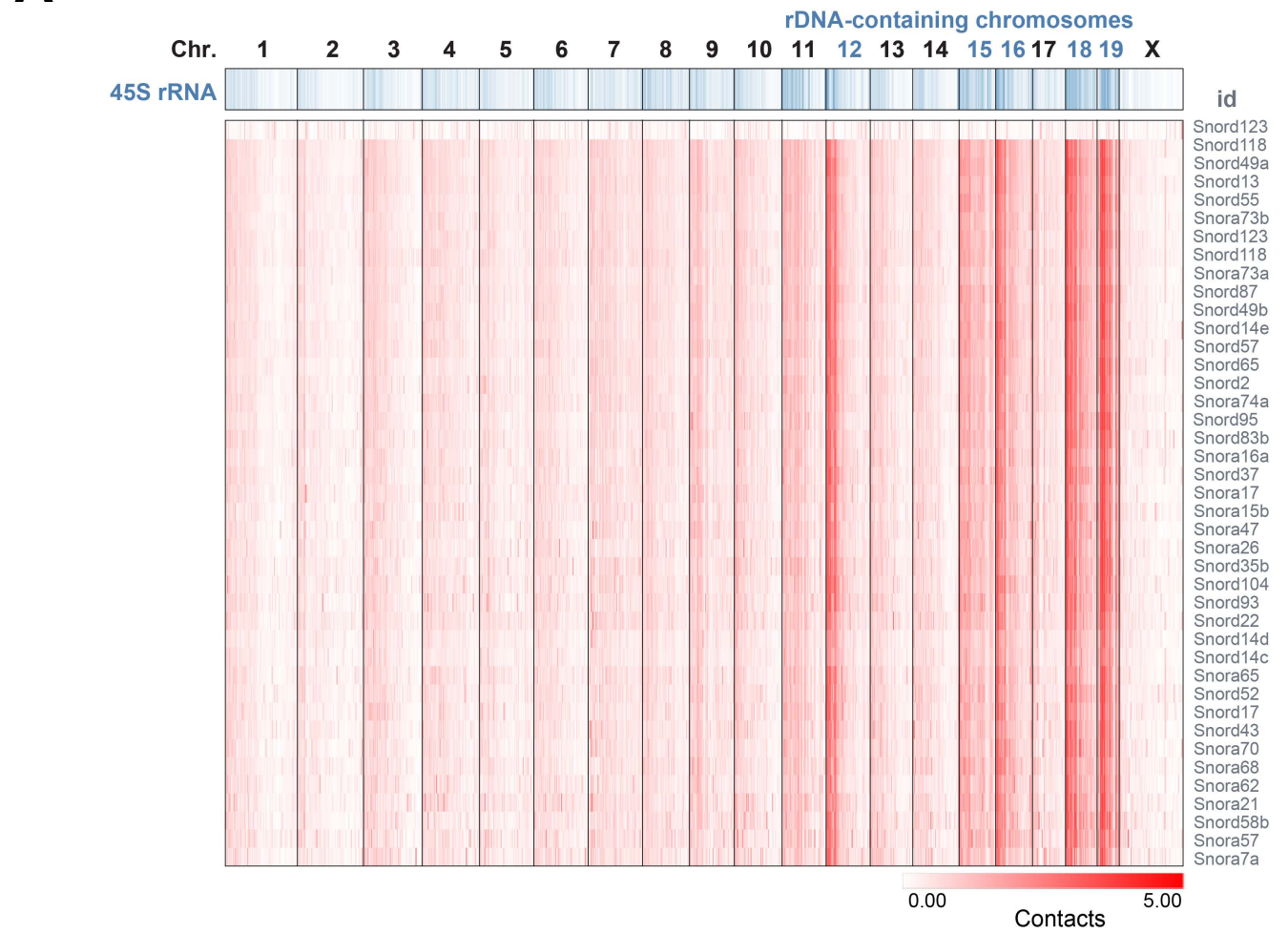

B
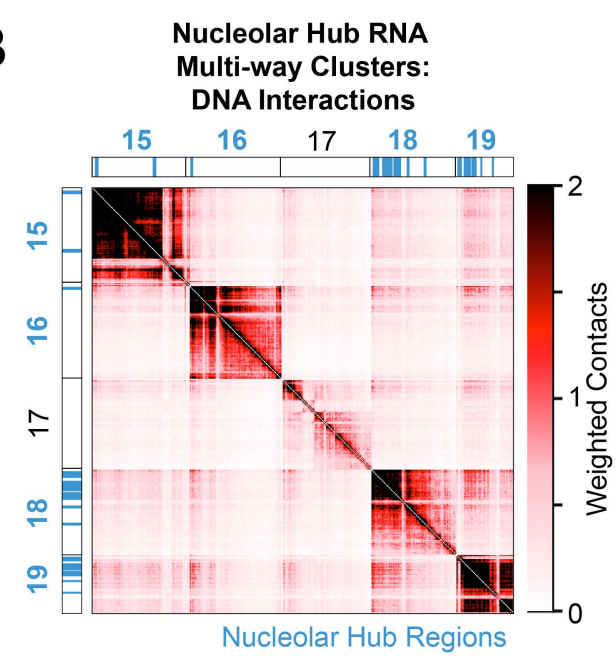

C
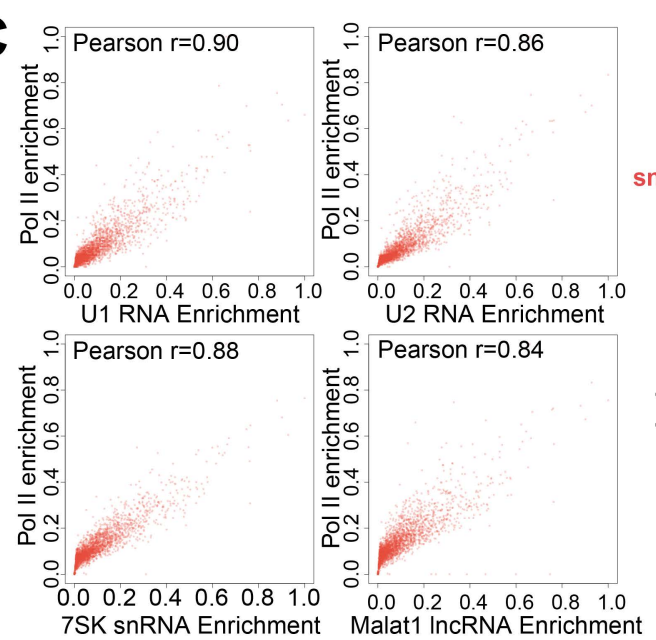
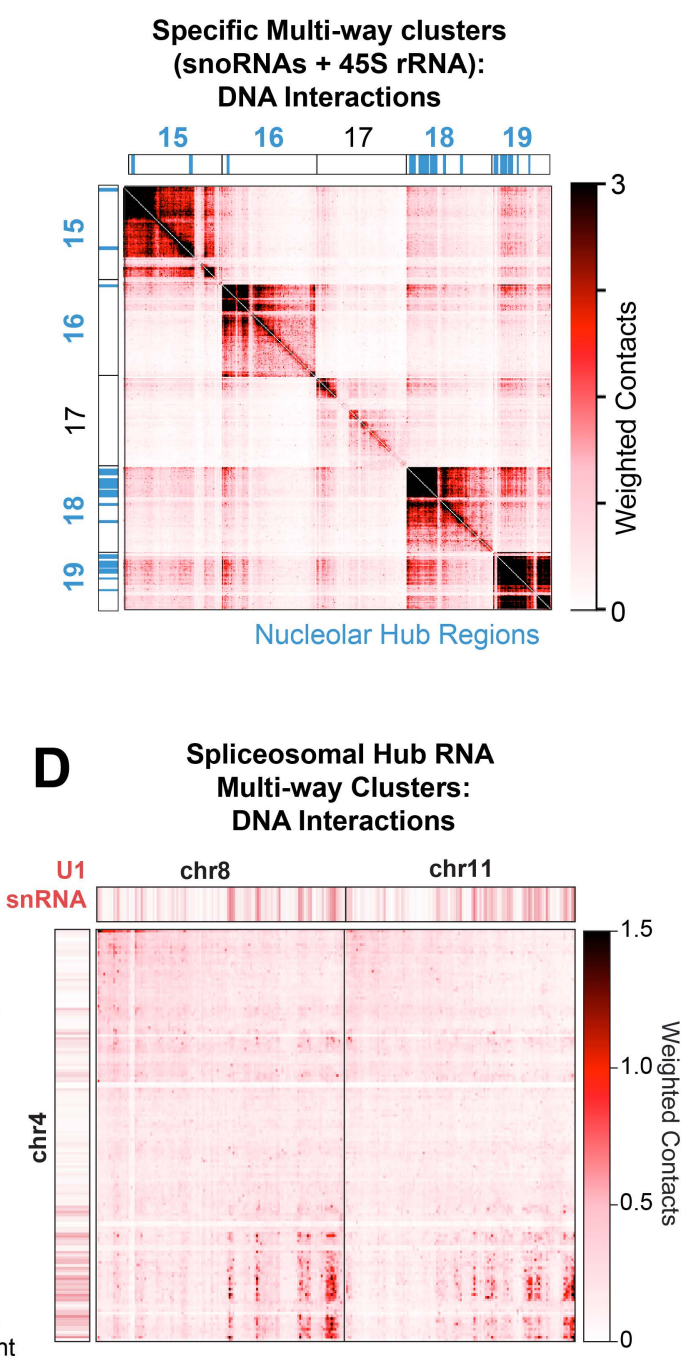

Specific Multi-way clusters (snoRNAs + 45SrRNA + 5S rRNA): DNA Interactions

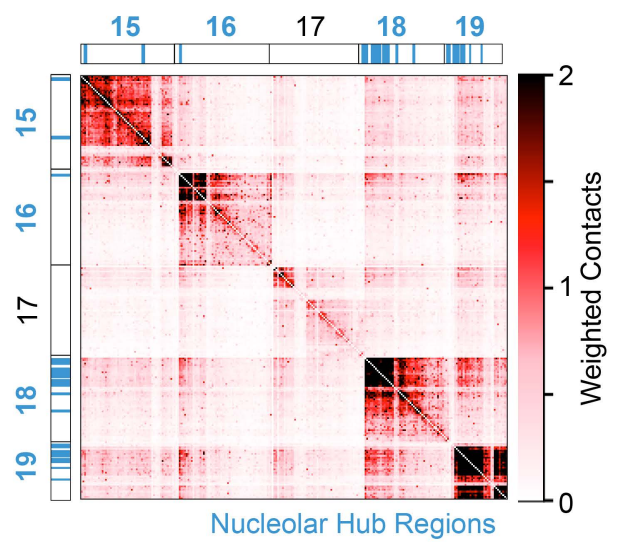

Spliceosomal Hub RNA clusters ( $\geq 2$ distinct RNAs/cluster): DNA Interactions chr8 $\quad$ chr11

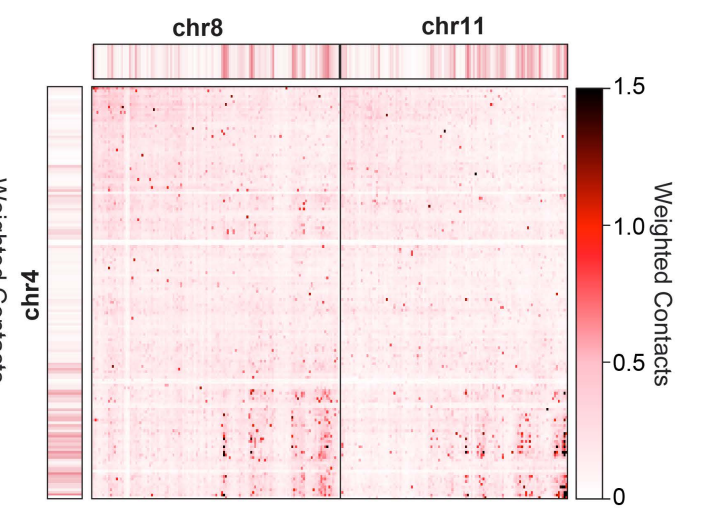


A $\square$ Antibody $\square$ DAPI
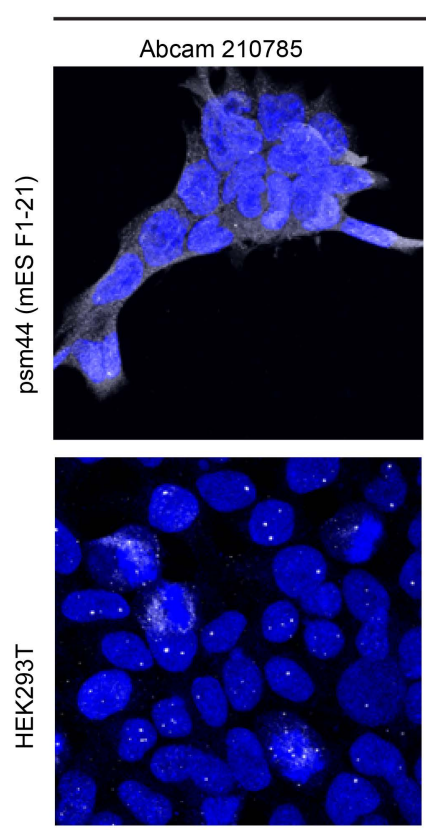

B
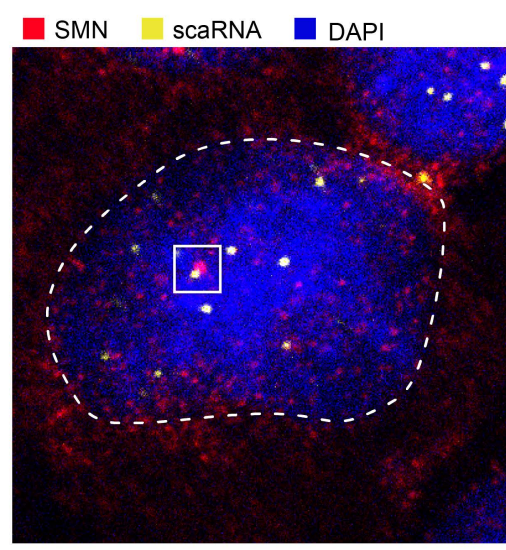

E
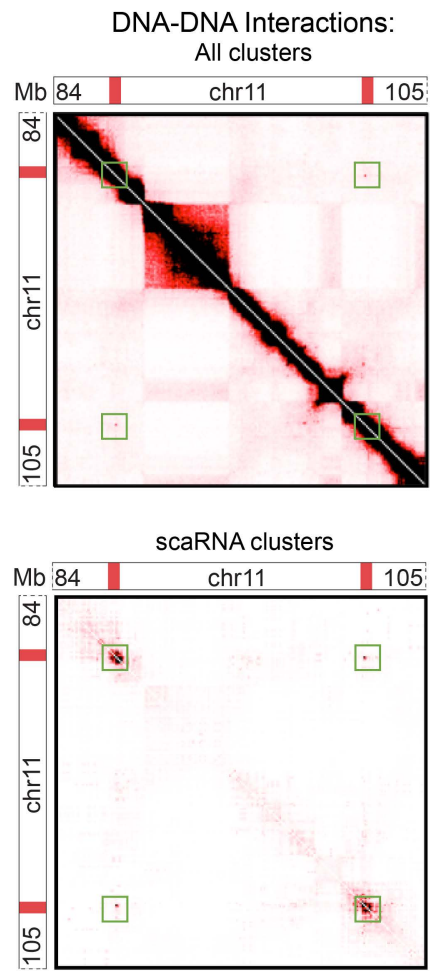

Anti-coilin
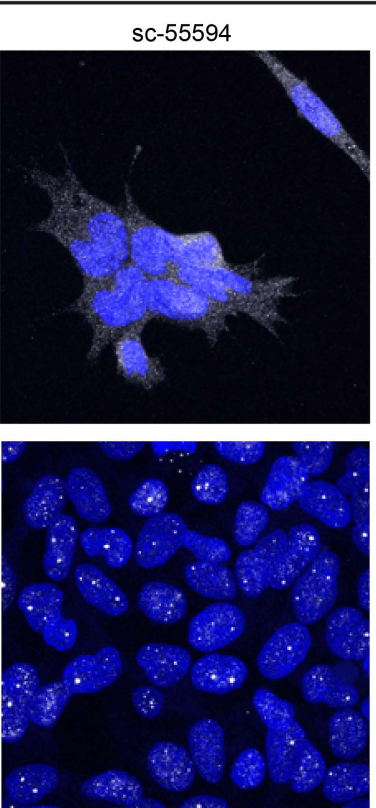

C

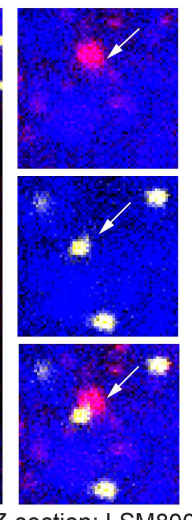

$\mathbf{F}$

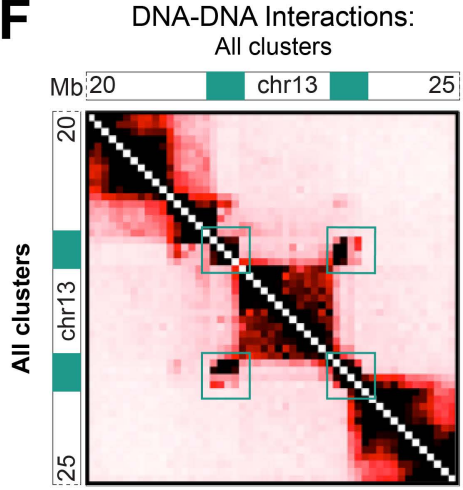

U7 RNA clusters

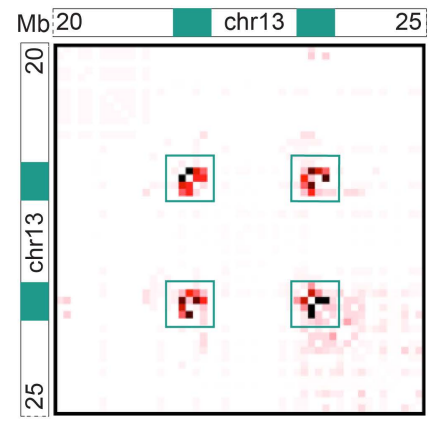

Sc-56298
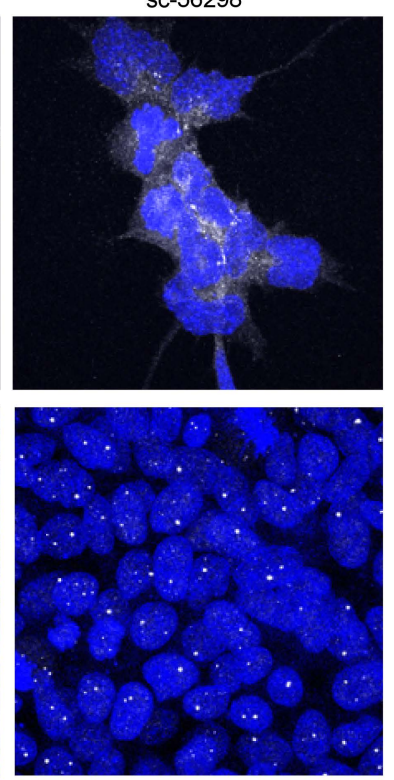

U $\square$ scaRNA DAPI
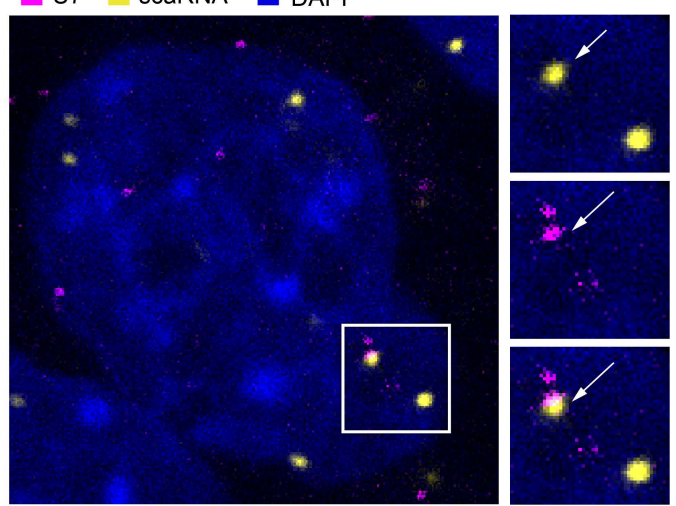

Z-section; LSM800

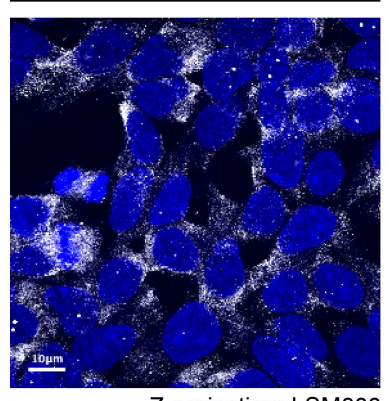

Z-projection; LSM800

D RNA-RNA Interactions

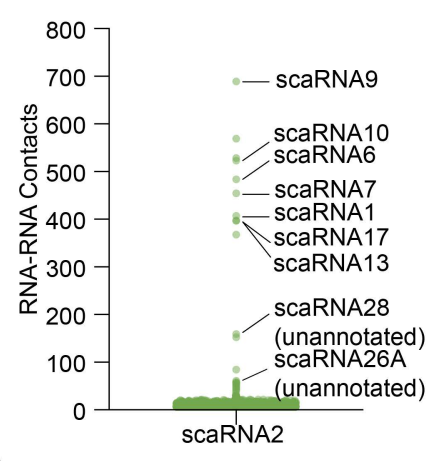

G DNA-DNA Interactions:

Multi-way clusters (scaRNAs + snRNAs)

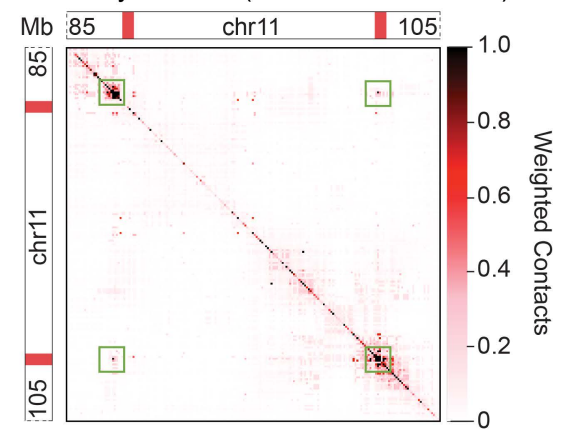

H DNA-DNA Interactions: scaRNA Clusters

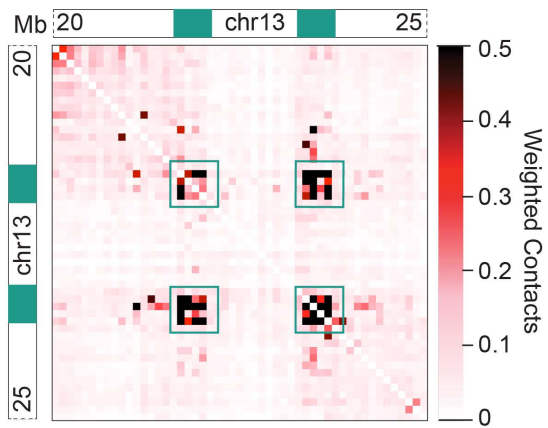


A

DNA-DNA Interactions: MinSat \& MajSat RNA Clusters

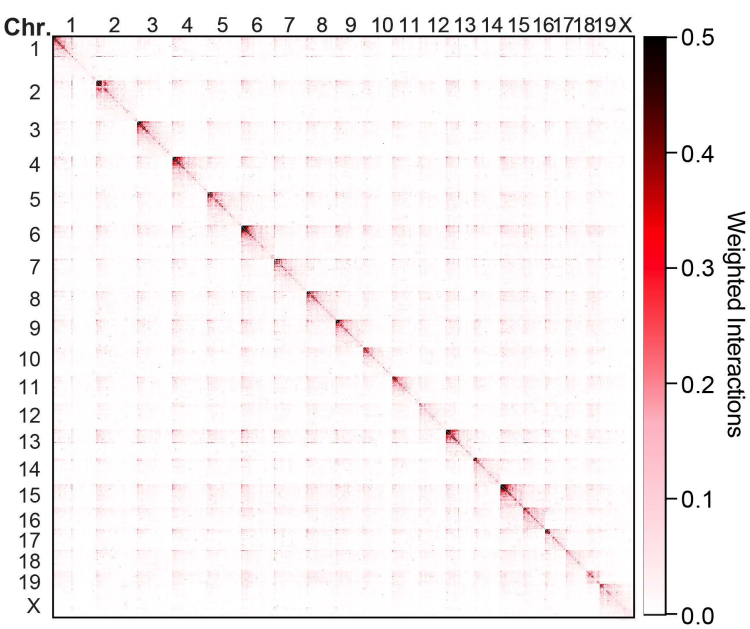

B

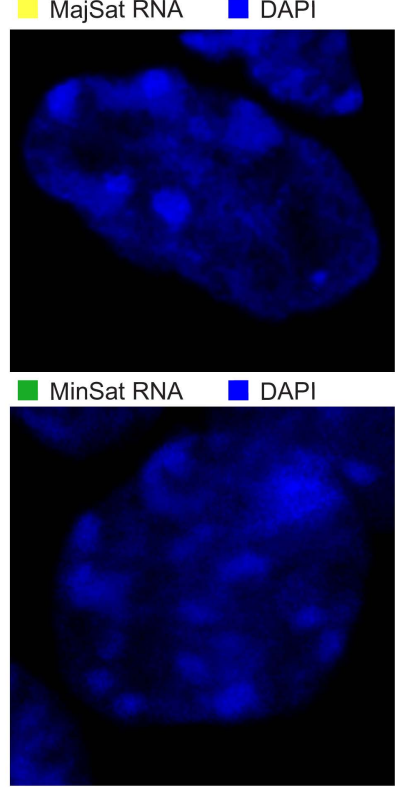

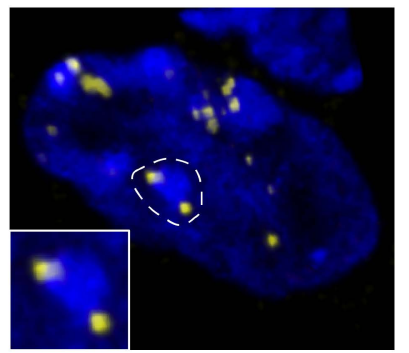

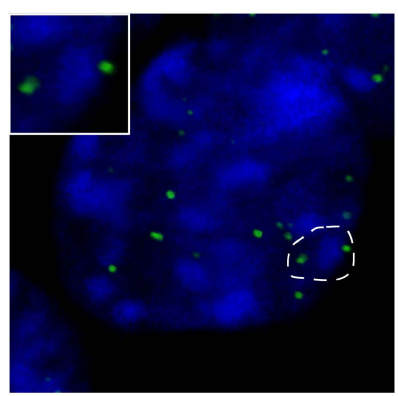

3D Projection; LSM800

C Minor Satellite LNA KD

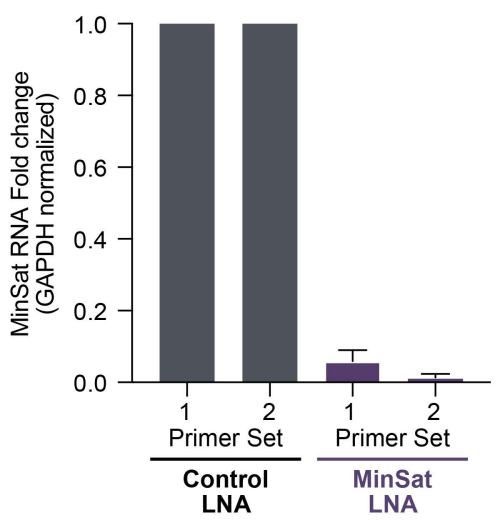

D

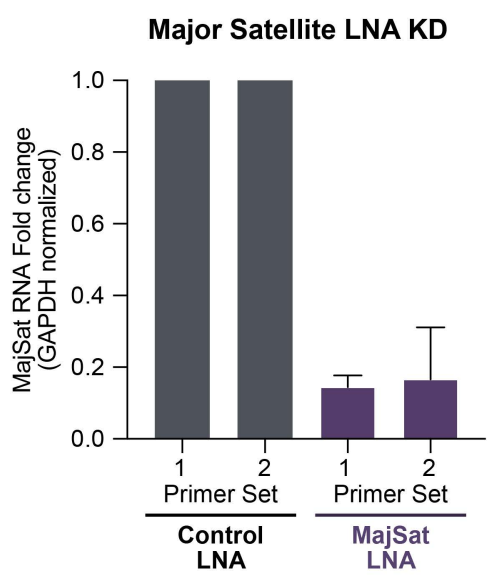

$\mathbf{F}$

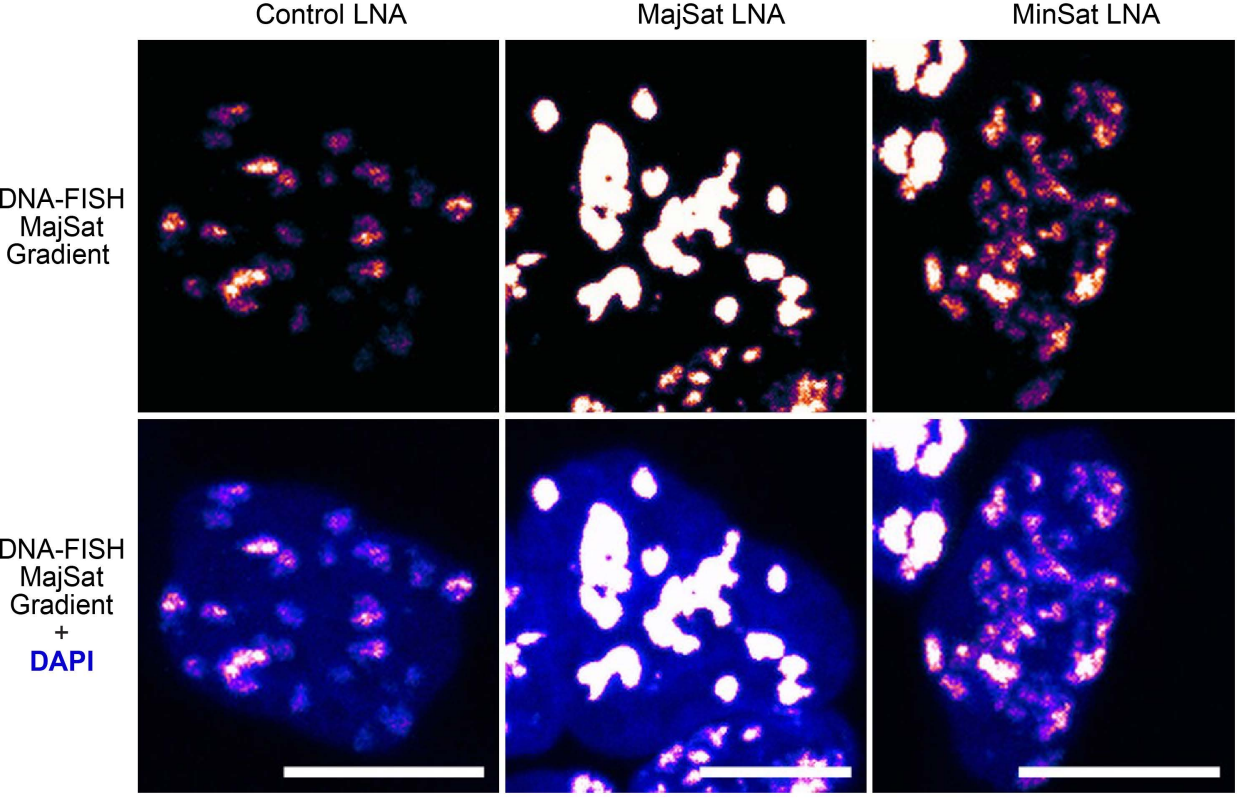

E

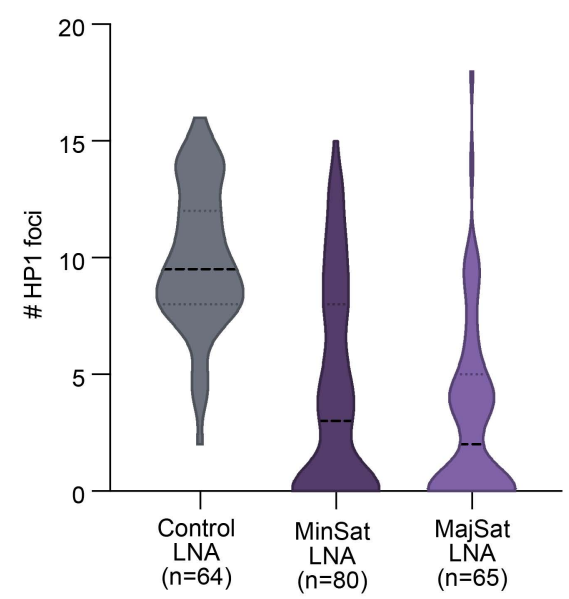


A

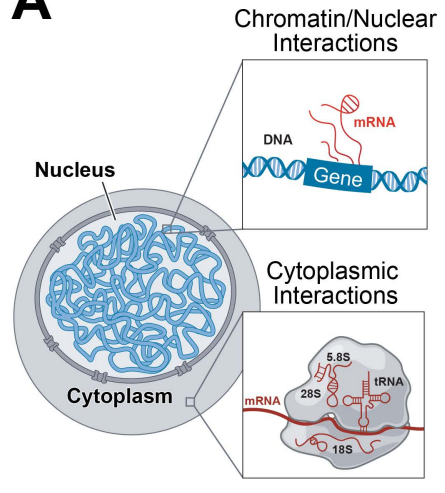

B

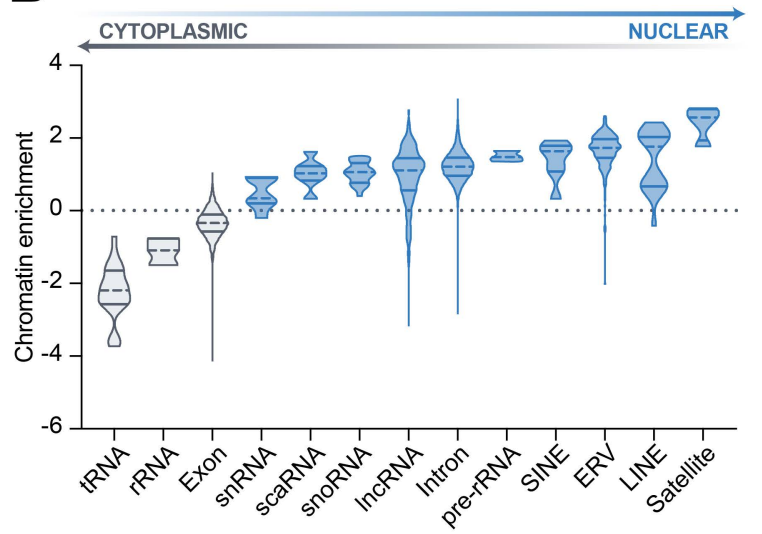

C

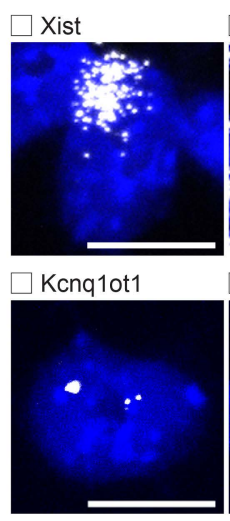

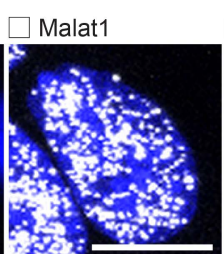

$\square$ Pvt1

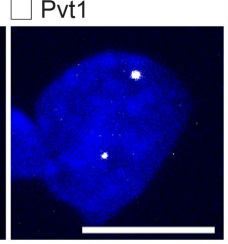

$\square$ Tsix

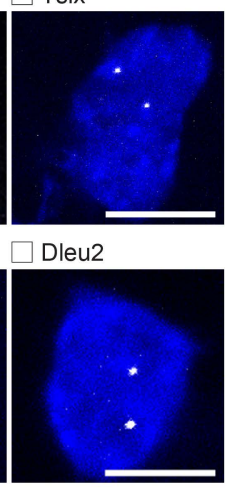

D

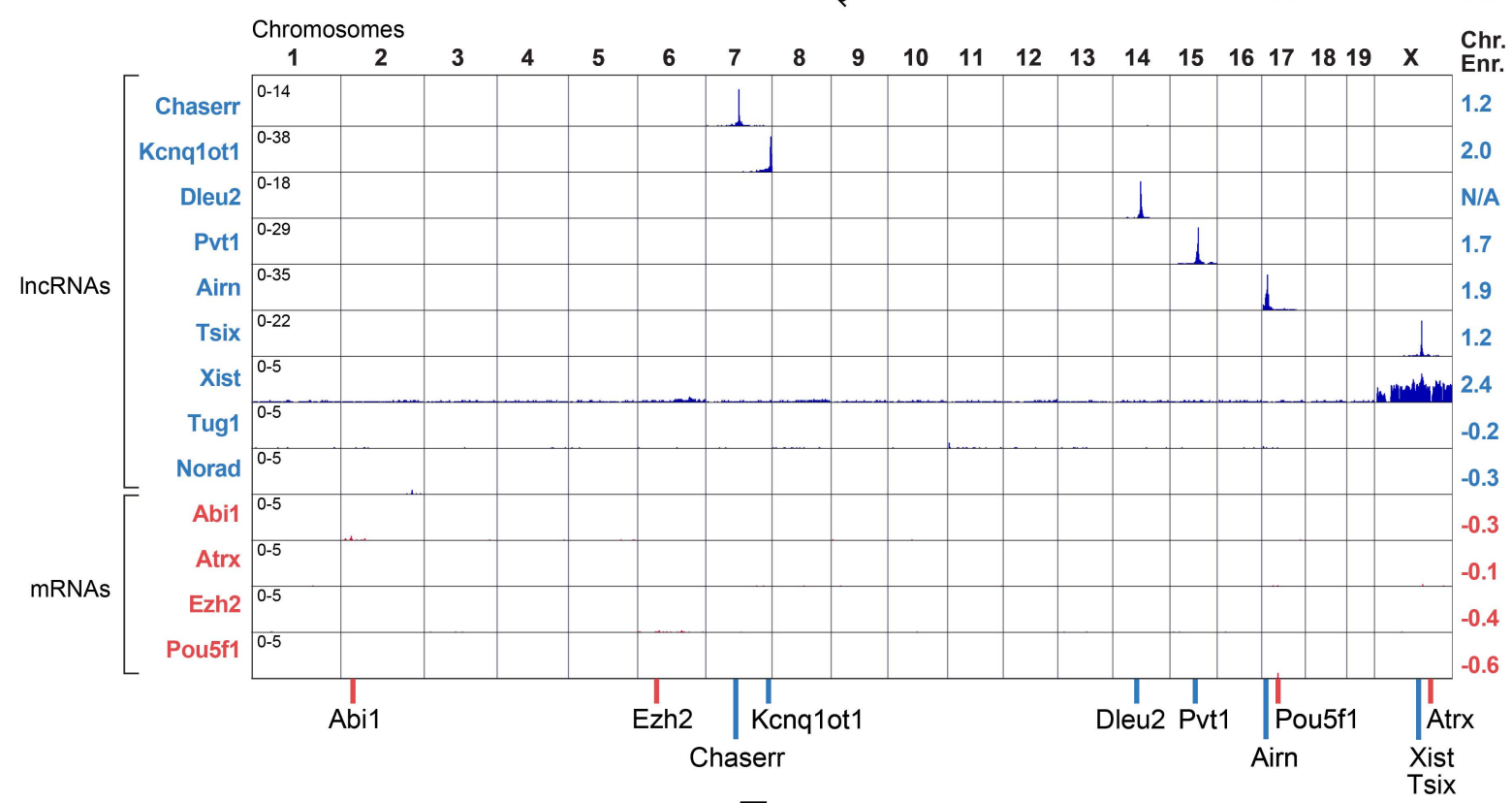

E

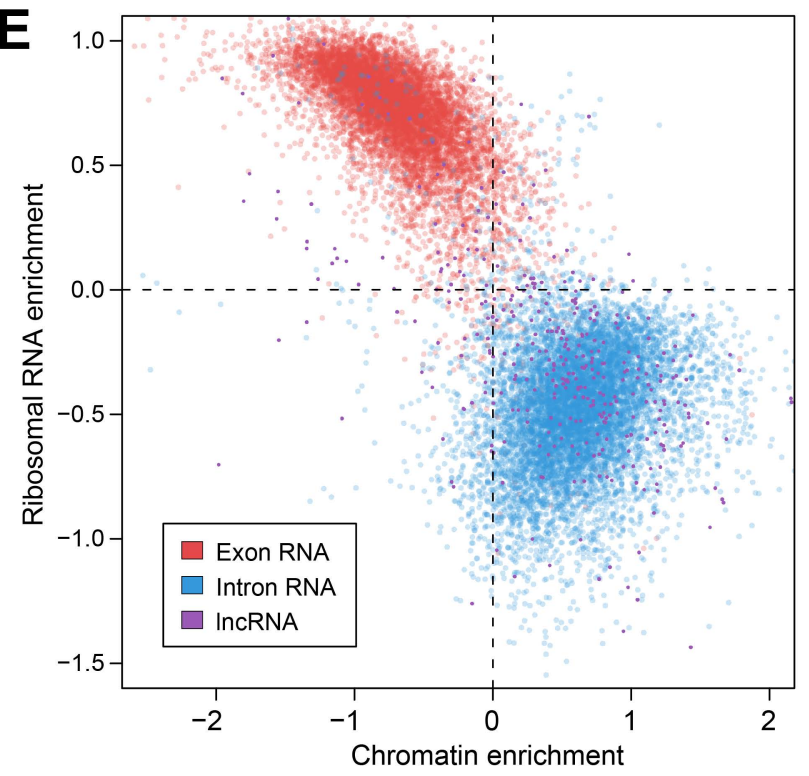

$F$
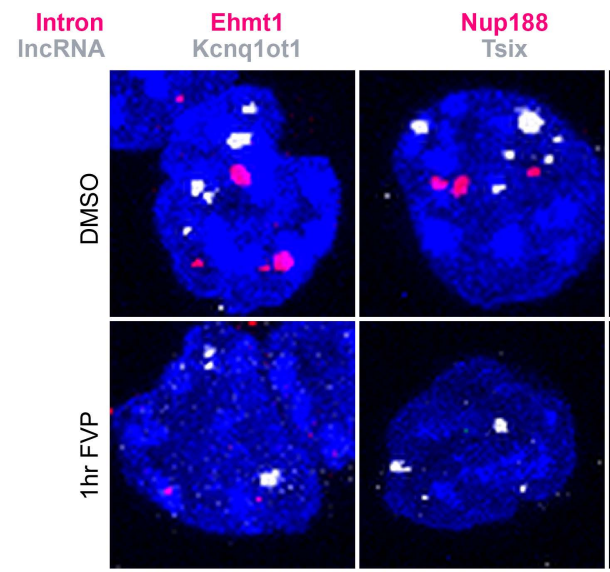
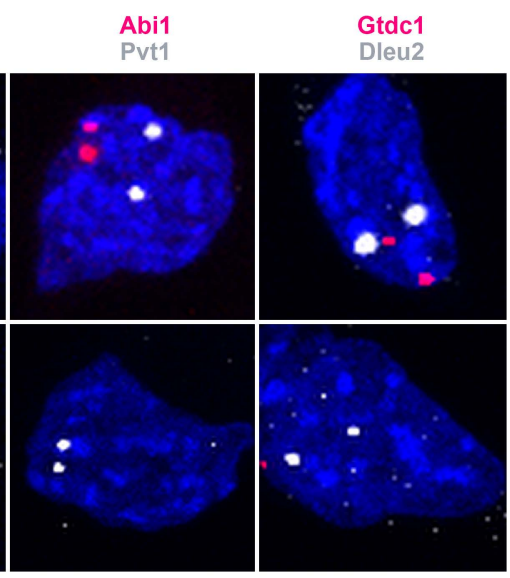

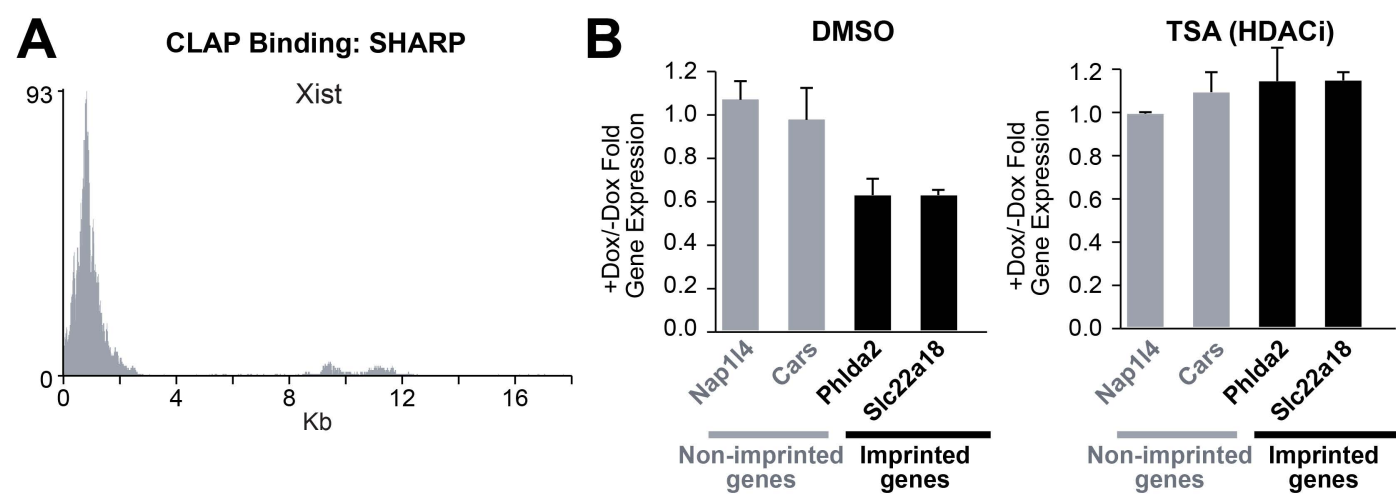
C Kcnq10t1 5' end Kcnq10t1 3' end DAPI

D Airn RNA Clusters:
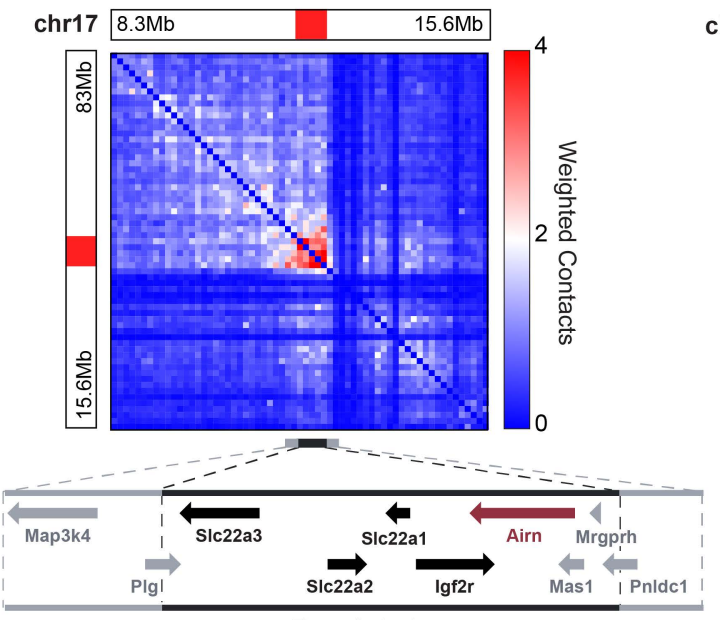

Regulated genes
E Pvt1 RNA Clusters:
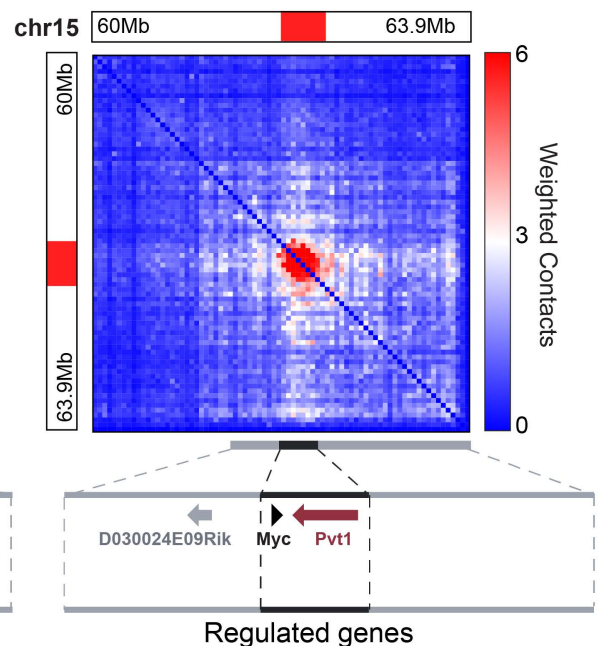

Regulated genes

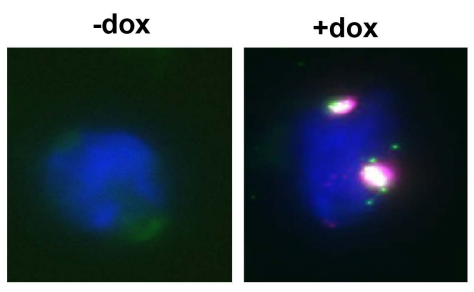

F Chaserr RNA Clusters:

DNA Interactions

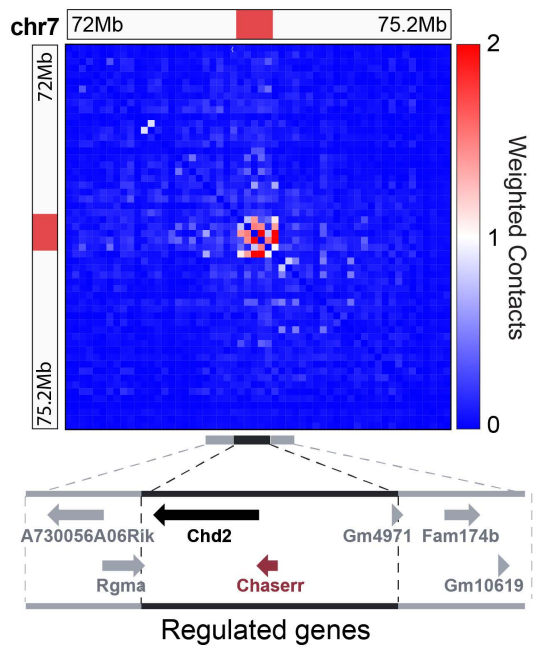

G
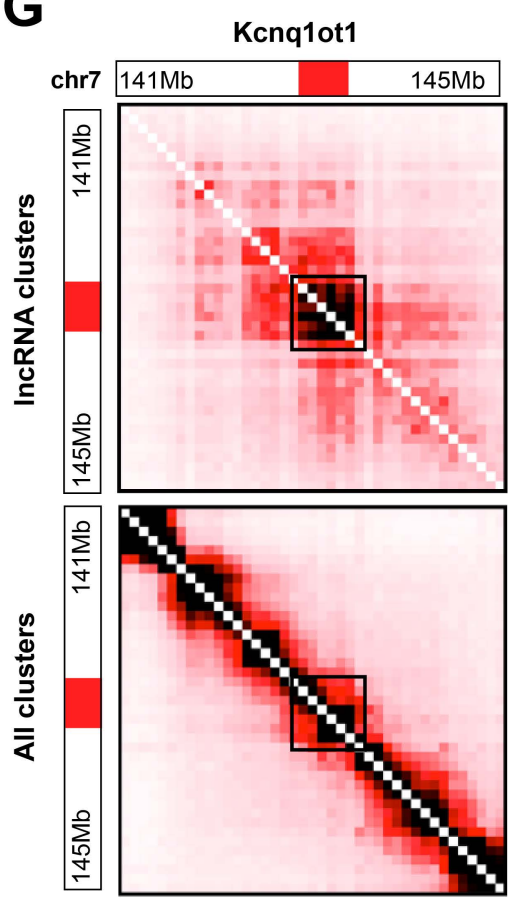

DNA Interactions

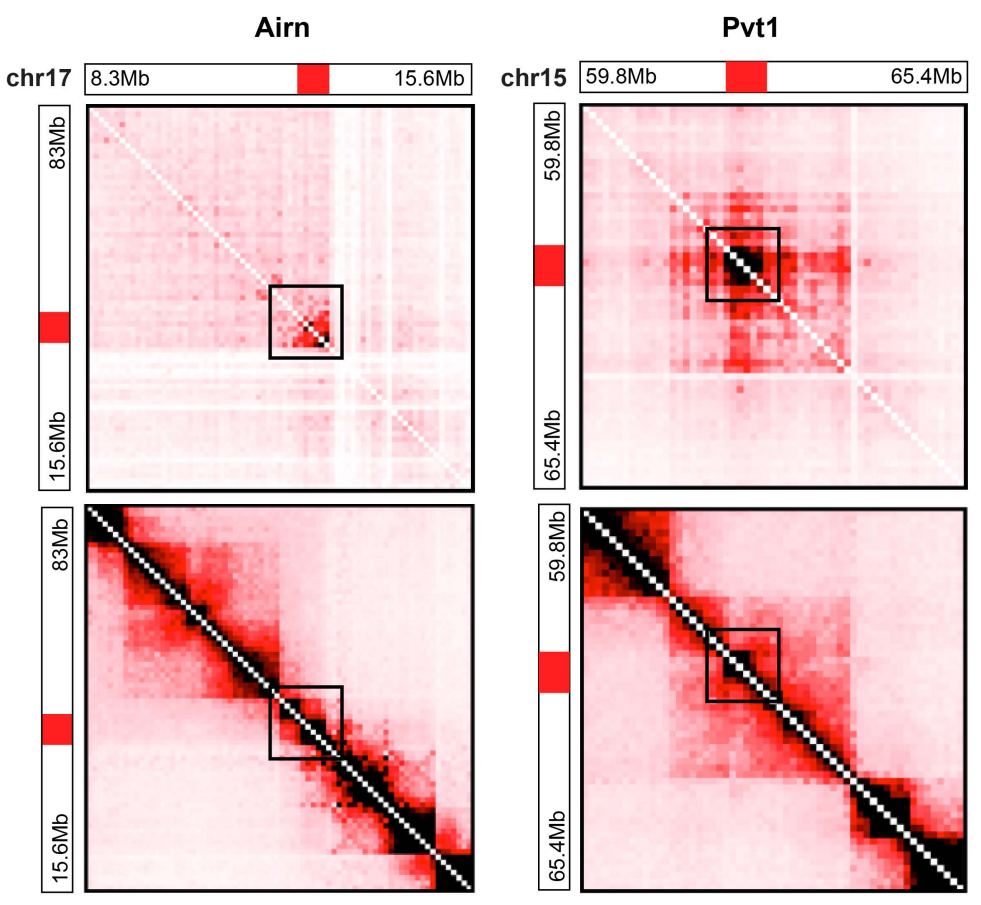


bioRxiv preprint doi: https://doi.org/10.1101/2020.08.25.267435. this version posted August 25, 2020. The copyright holder for this preprint (which was not certified by peer review) is the author/funder. All rights reserved. No reuse allowed without permission.

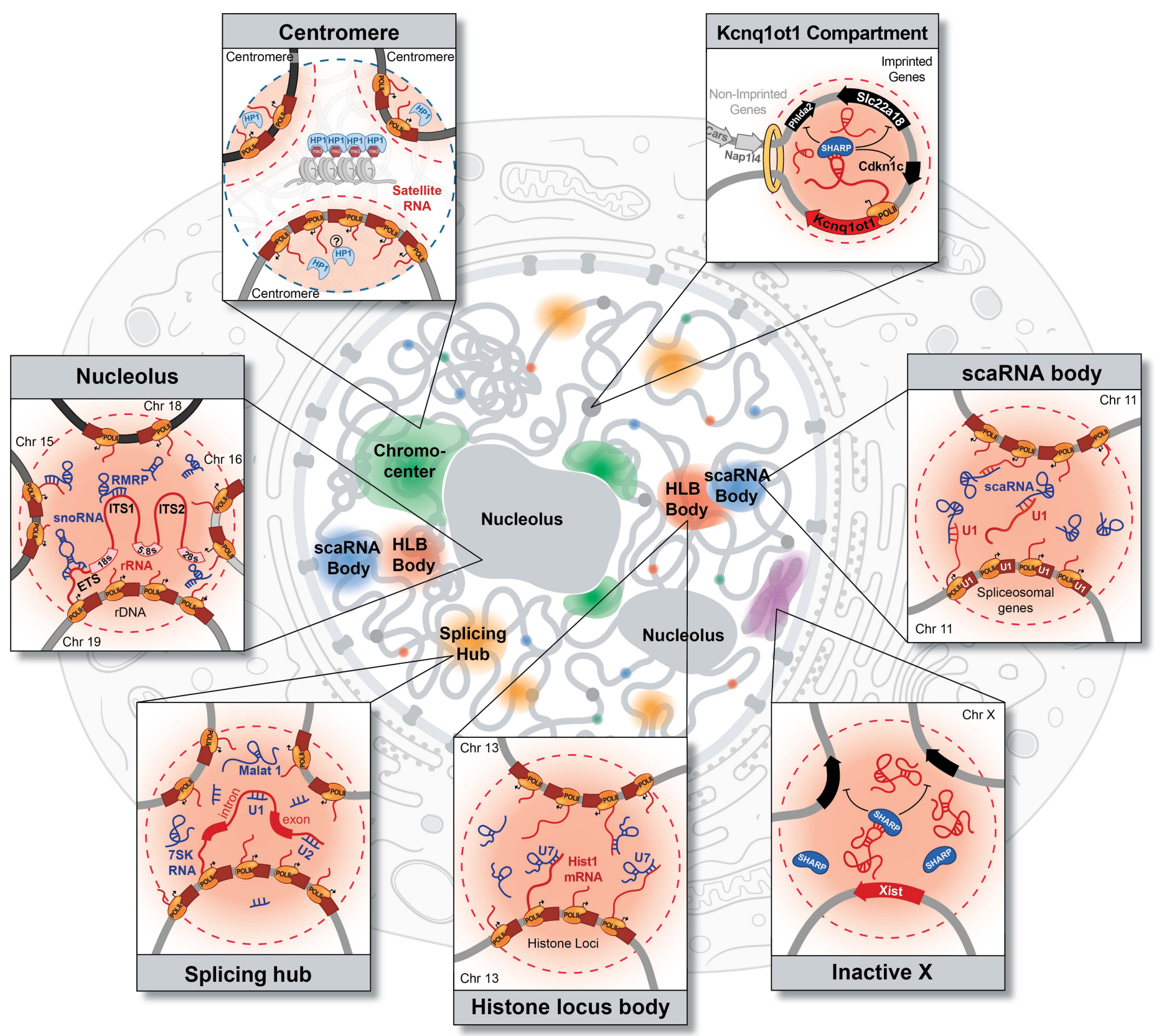

